\title{
Transversal Functional Analysis
}

\author{
Milan R. TASKOViĆ
}

\begin{abstract}
ShorT SURVEY. This paper provides an introduction to the ideas and methods of transversal functional analysis based on the transversal sets theory. A unifying concept that lies at the heart of transversal functional analysis is that of a transversal normed linear space. I have developed the theory far enough to include facts of have called the three new basic principles of linear analysis as: Form of Hahn-Banach theorem, Form of Principle of Uniform Boundedness (= Form of Banach-Steinhaus theorem), and Form of Open Mapping theorem. In the classical functional analysis fundamental fact is Riesz lemma. In transversal functional analysis (on lower transversal normed spaces) its role play so-called Geometrical lemma! This paper presents applications of the Axiom of Infinite Choice.
\end{abstract}

\section{Transversal UPPER NORMED SPACES}

Let $X$ be a linear space over $\mathbb{K}(:=\mathbb{R}$ or $\mathbb{C})$. The mapping $x \mapsto\|x\|$ : $X \rightarrow[a, b]$ for some $0 \leq a<b<+\infty$ or $x \mapsto\|x\|: X \rightarrow[a, b)$ for some $0 \leq a<b \leq+\infty$ is called an upper transversal seminorm (or upper seminorm) iff: $\|x\| \geq a$ for every $x \in X,\|\lambda x\|=|\lambda|\|x\|$ for all $\lambda \in \mathbb{K}$ and $x \in X$, and if there is a function $g:[a, b)^{2} \rightarrow[a, b)$ such that

$$
\|x+y\| \leq \max \{\|x\|,\|y\|, g(\|x\|,\|y\|)\}
$$

for all $x, y \in X$.

Further, $x \mapsto\|x\|$ is called an upper transversal norm (or upper norm) iff in addition: $\|x\|=a$ if and only if $x=0$.

An upper transversal normed space $(X,\|\cdot\|)$ over $\mathbb{K}$ consists of a linear space $X$ over $\mathbb{K}$ together with an upper transversal norm $x \mapsto\|x\|$.

2010 Mathematics Subject Classification. Primary: 04A25, 05A15, 47H10, 54E15; Secondary: 54H25, 54E24.

Key words and phrases. Transversal (upper, lower and middle) normed spaces, Transversal seminorms, Form of Hahn-Banach theorem, Form of Principle of Uniform Boundedness, Form of Banach-Steinhaus theorem, Form of Open Mapping theorem, Form of Riesz lemma, Geometrical lemma, Form of convergence principle, Lower compactness, Lower total continuous operators, Lower locally compactness, Lower Fredholm alternative, Extension Leray-Schauder principle, Peano's equation, Lower compact operators. 
The function $g:[a, b)^{2} \rightarrow[a, b)$ in (Nu) is called upper bisection function. From $(\mathrm{Nu})$ it follows, by induction, that there is a function $\mathfrak{M}$ : $[a, b)^{n} \rightarrow[a, b)$ such that

$$
\begin{gathered}
\left\|x_{0}-x_{n}\right\| \leq \\
\leq \max \left\{\left\|x_{0}-x_{1}\right\|, \ldots,\left\|x_{n-1}-x_{n}\right\|, \mathfrak{M}\left(\left\|x_{0}-x_{1}\right\|, \ldots,\left\|x_{n-1}-x_{n}\right\|\right)\right\}
\end{gathered}
$$

for all $x_{0}, x_{1}, \ldots, x_{n} \in X$ and for any fixed integer $n \geq 1$. (The proof of this fact it follows immediately from induction!)

It is easy to verify that every upper transversal normed linear space $X$ is a transversal upper space (see: Tasković [2005]) with respect to the upper transverse $\rho: X \times X \rightarrow \mathbb{R}_{+}^{0}$ defined by

$$
\rho[x, y]=\|x-y\| \quad \text { for all } \quad x, y \in X ;
$$

thus we obtain $\rho[x-z, y-z]=\rho[x, y]$ and $\rho[\lambda x, \lambda y]=|\lambda| \rho[x, y]$ for all $x, y, z \in X$ and for every scalar $\lambda \in \mathbb{K}$.

In this sense, an upper transversal normed space $X$ is said to be upper complete if it is upper complete as a transversal upper space. The upper convergence $x_{n} \rightarrow x(n \rightarrow \infty)$ means $\left\|x_{n}-x\right\| \rightarrow a(n \rightarrow \infty)$.

We will in further denote by $\mathfrak{G}([a, b])$ the set of all upper bisection functions $g:[a, b]^{2} \rightarrow[a, b]$ which are increasing satisfying $g(t, t) \leq t$ for every $t \in[a, b]$.

We notice that upper transversal norm $x \mapsto\|x\|$ is a general convex function. The proof is simple. See this chapter of the book.

Example 1 (Upper intervally normed space). Let $X$ be a linear space over $\mathbb{K}$ and let $\mathfrak{G}$ be the set of all nonincreasing, left continuous functions $x \mapsto M_{x}: \mathbb{R} \rightarrow[a, b]$ for $a<b\left(a, b \in \mathbb{R}_{+}^{0}\right)$, where $\inf M_{x}=a$ and $\sup M_{x}=b$. Then $(X, \mathfrak{G})$ is an upper transversal normed space if: $M_{x}(0)=a$ for every $x \in X, M_{x}=b$ for $x \leq 0$ and $M_{x}=a$ for $x>0$,

$$
M_{\lambda x}(\sigma)=M_{x}\left(\frac{\sigma}{|\lambda|}\right) \quad \text { for every } \quad x \in X
$$

and for all $\sigma \in \mathbb{R}$ and $\lambda \neq 0$, and if there is a function $g:[a, b]^{2} \rightarrow[a, b]$ for $a, b \in \mathbb{R}_{+}^{0}(a<b)$ such that the following ineuality holds in the form as

$$
M_{x+y}(p+q) \leq \max \left\{M_{x}(p), M_{y}(q), g\left(M_{x}(p), M_{y}(q)\right)\right\}
$$

for all $x, y \in X$ and for all $p, q \geq 0$.

The fundamental results of this section are the statements characterizing finite dimensional spaces (as a Riesz's theorem type), statement of separable upper transversal normed spaces and a statement as the form of Riesz lemma for upper transversal normed spaces. See brief proofs for this in: Tasković [2005].

Also, in this part, we derive a general extension theorem, as a form of Hahn-Banach theorem, for linear functionals on an arbitrary linear space. 
In the next we can apply this theorem to the problem of the existence of upper bounded linear functionals.

If in the preceding definition of transversal upper normed spaces the condition of homogenity of the form: $\|\lambda x\|=|\lambda|\|x\|$ for all $\lambda \in \mathbb{K}$ and $x \in X$ is to exchange with the condition of the form: $\|\lambda x\|=|f(\lambda)|\|x\|$ for all $\lambda \in \mathbb{K}$, $x \in X$, and a function $f: \mathbb{K} \rightarrow \mathbb{K}$, then we have a transversal upper global normed space $(X,\|\cdot\|)$, where $x \mapsto\|x\|$ is a transversal upper global norm. Adequate we obtain a transversal upper global semi-normed space as and transversal upper global seminorm.

On the other hand, in an upper transversal normed space $X$ with the upper bisection function $g \in \mathfrak{G}([a, b))$, the following inequality holds as

$$
\left\|\sum_{k=1}^{n} x_{k}\right\| \leq \sum_{k=1}^{n}\left\|x_{k}\right\|
$$

for all points $x_{1}, \ldots, x_{n} \in X$, or in a precisely form as the following inequality which is very practical in further as

$$
\left\|\sum_{k=1}^{n} x_{k}\right\| \leq \max \left\{\left\|x_{1}\right\|, \ldots,\left\|x_{n}\right\|\right\}
$$

for all points $x_{1}, \ldots, x_{n} \in X$. (The proof of inequalities (1) and (1') via induction may be found in: Task ović [2005].)

Otherwise, a fundamental first example of transversal upper normed spaces with the upper bisection function $g:\left(\mathbb{R}_{+}^{0}\right)^{2} \rightarrow \mathbb{R}_{+}^{0}$ defined by $g(s, t):=s+t$, is a classical normed space. ${ }^{1}$

Example 2 (The space $L_{p}(a, b)$ for $p \in \mathbb{R} \backslash\{0\}$ ). If in the set $L_{p}(a, b)$ for $p \in \mathbb{R} \backslash\{0\}$ introduced, in the classical style, the following compositions in the form as

$$
(x+y)(t)=x(t)+y(t), \quad \text { and } \quad(\lambda x)(t)=\lambda x(t),
$$

then we obtain that $L_{p}(a, b)$ is a vector space. The zero vector for this space is a function which is almost everywhere equel to zero. This space is a transversal

\footnotetext{
${ }^{1}$ History of normed spaces. J e a n D i e u d o n n é: "Starting from the work of Friedrich Riesz and $\mathrm{Eduard} \mathrm{Helly}$ on the problem of moments inertia it was a natural generalization to define norms on arbitrary vector spaces. This was done independently by $\mathrm{Stefan} \mathrm{B} \mathrm{a} \mathrm{n} \mathrm{c} \mathrm{h} \mathrm{and} \mathrm{H}$ an s $\mathrm{Hahn}$ about 1920. In 1932 Banach published a book containing a comprehensive account of all results known at that time in the theory of normed spaces, and in particular the theorems he had published in his papers of 1922 and 1929. A large part was devoted to the concept of weak convergence and its generalizations, which he had begun to study in 1929. This book had on Functional Analysis the same impact that Van der Waerden's book had on Algebra two years earlier. Analysts all over the world began to realize the power of the new methods and to apply them to a great variety of problems; Banach's terminology and notations were universally adopted, complete normed spaces became known as Banach spaces, and soon their theory was considered as a compulsory part in most curricula of graduate students. After 1935, the theory of normed spaces became part of the more general theory of locally convex spaces".
} 
upper normed space if the transversal upper norm $x \mapsto\|x\|$ is defined with

$$
\|x\|=\left(\int_{a}^{b}|x(t)|^{p} d t\right)^{1 / p}
$$

for every $x \in L_{p}(a, b)$ and for an arbitrary fixed $p \in \mathbb{R} \backslash\{0\}$. Then to arise that (2) has all attributes of transversal upper norm, where inequality $(\mathrm{Nu})$ is the form as

$$
\|x+y\| \leq \max \left\{\|x\|,\|y\|,\left(\int_{a}^{b}(|x(t)|+|y(t)|)^{p} d t\right)^{1 / p}\right\}
$$

for all $x, y \in L_{p}(a, b)$ and $p \in \mathbb{R} \backslash\{0\}$. (We notice, in the special case for $p \geq 1$, the inequality $(\mathrm{Nu})$ may be in something differently form.)

Something dilatation. (The spaces $L_{p}$ for $p \in \mathbb{R} \backslash\{0\}$ ). Let $\Omega$ be a nonempty set and let $\Sigma$ be a $\sigma$-algebra subsets of $\Omega$. Suppose that $\mu$ is a nonnegative, nontrivial, complete, and $\sigma$-finite measure on $\Omega$. Let $(\Omega, \Sigma, \mu)$ be a measure space and $p \in \mathbb{R} \backslash\{0\}$. An $L_{p}$ space essentially consists of all measurable functions $x$ such that $\|x\|^{p}$ is an integrable function with (2) taken as the upper transversal norm.

The case $0<p<1$. In the case $x \in L_{p}$ for $0<p<1$ the function (2) is an upper trahsversal norm. But, an upper transversal norm $f \mapsto\|f\|$ we can defined and with

$$
\|f\|=\int_{\Omega}|f|^{p} d \mu \quad \text { for } \quad 0<p<1 ;
$$

where all properties of the upper transversal norm hold and where inequality $\mathrm{Nu}$ ) holds from the following inequality in the from as

$$
(x+y)^{p} \leq x^{p}+y^{p} \quad \text { for } \quad 0<p<1
$$

and for all $x, y \geq 0$. Otherwise, this form of inequality $(\mathrm{Nu})$ via (3) has many applications in mathematical analysis.

We notice that every upper intervally normed space is a transversal upper intervally space if a transverse $\rho[x, y]=M_{x-y}$. Let $\tau(G)$ be a topology for $a \geq 0$ in the following sense as

$$
G_{x}(\varepsilon)=\left\{y \in X: M_{x-y}(\varepsilon)<a+\varepsilon\right\}
$$

for every $x \in X$ and for every $\varepsilon>0$. In the next set $A(x)=b$ for $x \leq 0$ and $A(x)=a$ for $x>0$. Now we can formulate the following result.

Proposition 1. Let $X$ be a vector space over $\mathbb{K}(:=\mathbb{R}$ or $\mathbb{C}), M_{x}(0)=$ a for every $x \in X, M_{x}=A$ if and only if $x=0, M_{\alpha x}(\varepsilon) \geq M_{x}(\varepsilon)$ for $0<|\alpha|<1$, $M_{\alpha x}(\varepsilon) \rightarrow a$ as $\alpha \rightarrow 0$, and

$$
M_{x-y}(\varepsilon)<a+\varepsilon \quad \text { and } \quad M_{y-z}(\delta)<a+\delta \text { implies } M_{x-z}(\varepsilon+\delta)<a+(\varepsilon+\delta)
$$


for all $\varepsilon, \delta>0$, then $(X, \tau(G))$ is a transversal topological vector space, where the upper transverse $\rho$ has the following form as

$$
\rho[x, y]= \begin{cases}0 \text { if } y \in G_{x}(\varepsilon) & \text { for every } \varepsilon>0, \\ \inf \left\{\varepsilon: y \notin G_{x}(\varepsilon)\right\} & \text { for every } 0<\varepsilon<1,\end{cases}
$$

in agreement with translation and invariance of the upper transverse in the form: $\rho[0, \lambda x] \leq \rho[0, x]$ for $|\lambda|<1$. Also, $\rho[x, y]>\tau$ if and only if $M_{x-y}(\tau)<a+\tau$ for every $\tau>0$.

Proof. Set $\mathfrak{D}=\left\{G_{x, y}=M_{x-y}: x, y \in X\right\}$, then directly, from the preceding facts we obtain the following fact that

$$
G_{x, y}(\varepsilon)<a+\varepsilon \text { and } G_{y, z}(\delta)<a+\delta \text { implies } G_{x, z}(\varepsilon+\delta)<a+(\varepsilon+\delta)
$$

for all $\varepsilon, \delta>0$. On the other hand, the condition (5) implies the following condition in the form as: for every $\varepsilon>0$ there exists $0<\delta \leq \varepsilon$ such that

$$
G_{x, y}(\delta)<a+\delta \text { and } G_{y, z}(\delta)<a+\delta \text { implies } G_{x, z}(\varepsilon)<a+\varepsilon,
$$

where the condition (6) is to equivalent with the fact that $(X, \tau(G))$ is a topological vector space, i.e., the first part of statement holds. Also, from the fact

$$
\rho[0, x-z]=\inf \left\{\varepsilon: M_{0-(x-z)}(\varepsilon)=M_{x-z}(\varepsilon) \geq a+\varepsilon\right\}=\rho[x, z],
$$

it follows that $\rho[x+y, y+z]=\rho[0, x+y-(y+z)]=\rho[0, x-z]=\rho[x, z]$. In this context, for $|\lambda| \leq 1$ the following fact holds as

$$
\rho[0, \lambda x]=\inf \left\{\varepsilon: M_{\lambda x}(\varepsilon) \geq a+\varepsilon\right\} \leq \inf \left\{\varepsilon: M_{x}(\varepsilon) \geq a+\varepsilon\right\}=\rho[0, x],
$$

i.e., from these facts we obtain that the upper transverse is invariance and translation. Also holds and second part of statement because $\tau(G)=\tau(\mathfrak{D})$. The proof is complete.

We notice that if $(X, \mathfrak{D})$ is an upper transversal intervally normed space with an upper bisection function $g \in \mathfrak{D}([a, b])$, then (4) holds, because for $M_{x-y}(\varepsilon)<a+\varepsilon$ and $M_{y-z}(\delta)<a+\delta$ we obtain that

$$
\begin{gathered}
M_{x-z}(\varepsilon+\delta)=M_{x-y+y-z}(\varepsilon+\delta) \leq \\
\leq \max \left\{M_{x-y}(\varepsilon), M_{y-z}(\delta), g\left(M_{x-y}(\varepsilon), M_{y-z}(\delta)\right)\right\} \leq \\
\leq \max \left\{M_{x-y}(\varepsilon), M_{y-z}(\delta), g\left(\max \left\{M_{x-y}(\varepsilon), M_{y-z}(\delta)\right\}, \max \left\{M_{x-y}(\varepsilon), M_{y-z}(\delta)\right\}\right)\right\} \leq \\
\leq \cdots \leq \max \left\{M_{x-y}(\varepsilon), M_{y-z}(\delta), \max \left\{M_{x-y}(\varepsilon), M_{y-z}(\delta)\right\}\right\}< \\
<\max \{a+\varepsilon, a+\delta, \max \{a+\varepsilon, a+\delta\}\}<a+(\varepsilon+\delta) .
\end{gathered}
$$


The space $B(S)$. The upper transversal normed space of primary interest to us is the space $B(S)$ of all upper bounded scalar-valued functions $x: S \rightarrow \mathbb{R}$ defined on an arbitrary set $S$, with the upper norm given by

$$
\|x\|=\sup \{|x(t)|: t \in S\} .
$$

If the upper bisection function is defined by $g(s, t):=s+t$ and $S=$ $\{1,2, \ldots, p\}$, then $B(S)$ is the space $\mathbb{R}^{n}$ with the upper norm $\|\cdot\|_{\infty} ;$ and if $S=\mathbb{N}$, then we obtain the upper transversal normed space $B(\mathbb{N})=m$ consists of all upper bounded sequences.

Annotation. Many important properties of upper transversal normed spaces (an example of a Banach space) are closely linked to the shape of its closed unit sphere, that is, $g(S(0,1)):=\{x:\|x\|=1\}$. One basic property of $g(S(0,1))$ is that it is always general convex (see chapter 5 ). Let our underlying linear space be the real linear space $\mathbb{R}^{2}$ of all ordered pairs $x=\left(x_{1}, x_{2}\right)$ of real numbers. As on Figure 1 we have seen, there are many different upper norms which can be defined on $\mathbb{R}^{2}$, among which are the following: $\|x\|_{1}=\left|x_{1}\right|+\left|x_{2}\right|,\|x\|_{2}=\left(\left|x_{1}\right|^{2}+\left|x_{2}\right|^{2}\right)^{1 / 2}$, and $\|x\|_{\infty}=\max \left\{\left|x_{1}\right|,\left|x_{2}\right|\right\}$. Figure 1 illustrates the closed unit sphere which correspondes to each of these upper norms.

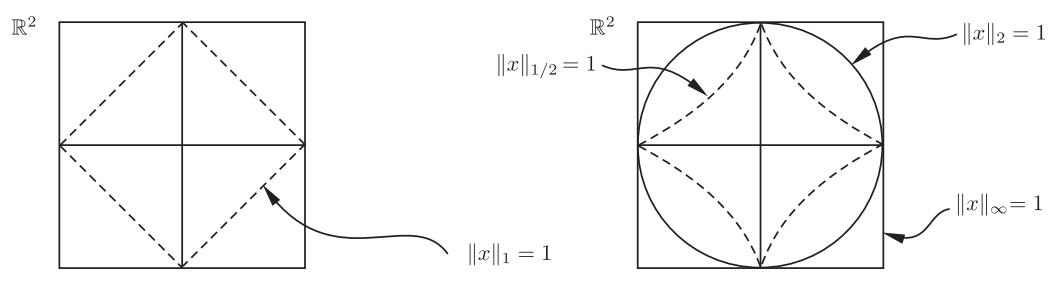

Figure 1. Some closed unit spheres

Proposition 2. Let $S$ be an arbitrary set and let $B(S)$ be an upper transversal linear normed space of all upper bounded real functions $x: S \rightarrow \mathbb{R}$ with the upper norm $\|x\|=\sup \{|x(t)|: t \in S\}$. Then $B(S)$ is an upper complete space.

The proof of this statement may be found (on a standard adequate manner) in: Tasković [2005].

We notice, in general case, that the upper transversal norm is not a continuous function. But, if the bisection function is $g \in \mathfrak{G}\left(\mathbb{R}_{+}^{0}\right)$, then upper norm is a continuous (even and uniformly continuous) function from the inequality

$$
|\|x\|-\|y\|| \leq\|x-y\| \quad \text { for all } \quad x, y \in X .
$$


Proposition 3. Let $X$ be a linear space and let $(X, \rho)$ be an upper transversal space, then upper transverse $\rho$ can be defined with upper norm if and only if the following facts hold:

$$
\begin{gathered}
\rho[x-a, y-a]=\rho[x, y] \quad \text { for all } \quad x, y, a \in X, \\
\rho[\lambda x, \lambda y]=|\lambda| \rho[x, y] \quad \text { for all } \quad x, y \in X
\end{gathered}
$$

and for every $\lambda \in \mathbb{K}(:=\mathbb{R}$ or $\mathbb{C})$.

Proof. Let $f: X \rightarrow \mathbb{R}$ be a defined by $f(x)=\rho[0, x]$ for $x \in X$, then $f$ is an upper transversal norm on $X$. Indeed, let $\lambda \in \mathbb{K}$ and $x \in X$, then from (8) it follows that

$$
f(\lambda x)=\rho[0, \lambda x]=\rho[\lambda 0, \lambda x]=|\lambda| \rho[0, x]=|\lambda| f(x),
$$

i.e., $f$ is an absolute homogeneous function. On the other hand, from (7) and given conditions, it follows that for all $x, y \in X$ we have the following inequality in the form

$$
\begin{gathered}
f(x+y)=\rho[0, x+y]=\rho[-x, y] \leq \\
\leq \max \{\rho[-x, 0], \rho[0, y], g(\rho[-x, 0], \rho[0, y])\}=\max \{f(x), f(y), g(f(x), f(y))\},
\end{gathered}
$$

which means that the function $f$ satisfy the correspondent inequality for upper transverse, i.e., $f$ is an upper (transversal) norm on $X$. Otherwise,

$$
\rho[x, y]=\rho[0, y-x]=f(y-x) \quad \text { for all } \quad x, y \in X,
$$

and thus, from the fact (8), it follows that $\rho[x, y]=f(x-y)$, i.e., the upper transverse $\rho$ is defined with the upper norm $f$. The proof is complete.

An annotation. We notice that a special form of the preceding Proposition 3, for metric linear spaces, is proved in 1936 by $\mathrm{S}$. K a k ut a n i on a different manner; see: R o le w i c z [1984].

Further facts. Also, with the same suppositions and $g \in \mathfrak{G}([a, b))$, if $E$ is a real (or complex) transversal upper normed space, the mapping $(x, y) \mapsto x+y$ is uniformly continuous in $E \times E$. The mapping $(\lambda, x) \mapsto \lambda x$ is continuous in $\mathbb{R} \times E$ (or $\mathbb{C} \times E$ ); the mapping $x \mapsto \lambda x$ is uniformly continuous in $E$.

As a corollary of this facts it follows that any translation $x \mapsto a+x$ and any homothetic mapping $x \mapsto \lambda x(\lambda \neq 0)$ is a homeomorphism of $E$ onto itself, for the inverse mapping is again a translation (resp. a homothetic mapping). For every vector $a \neq 0$ in $X$ the mapping $\lambda \mapsto \lambda a$ is a homeomorphism of the field $\mathbb{R}$ on the line $\mathbb{R} a$ in $X$.

Every two open (closed) balls, as and every two spheres, in a transversal upper normed space $X$ are homeomorphic. Unhurt space, also, is homeomorphic to an arbitrary proper open ball. Thus in $X$ are essential the balls and spheres with center 0 and radius 1 , i.e.,

$$
g(K(0,1)), g(K[0,1]), \text { and } g(S(0,1)) .
$$

If the upper bisection function $g \in \mathfrak{G}([a, b))$, then every ball is a convex set; also, for different of upper transversal spaces, we have that $\mathrm{Cl}(g(K(a, r)))=g(K[a, r])$ and $\operatorname{Int}(G(K[a, r]))=g(K(a, r))$ for $a \in X$ and $r>0$. 
If $x \mapsto\|x\|$ is an upper norm on the vector space $E$, then $\rho[x, y]=\|x-y\|$ is a transverse on $E$ such that $\rho[x+z, y+z]=\rho[x, y]$ and $\rho[\lambda x, \lambda y]=|\lambda|[x, y]$ for any scalar $\lambda \in \mathbb{K}$.

The verification of the axioms of transversal upper spaces is trivial. An upper transversal normed space is a vector space $X$ with a given upper norm on $X$; such a space is always considered as a transversal upper space for the transverse $\|x-y\|$.

Let $F$ be a linear subspace of a linear space $E$. The linear space $E / F:=\{x+F$ : $x \in E\}$ for $x+F:=\{x+y: y \in F\}$ for all $x \in E$. The sets $x+F$ are called cosets of $F$ in $E$. We observe that $F=0+F$. The linear space $E / F$ defined above is called the quotient space of $E$ modulo $F$. The mapping $x \mapsto x+F$ of $E$ onto $E / F$ is called the canonical mapping.

The realationship between the quotient space $E / F$ and linear subspaces of $E$ complementary to $F$ is simple. Let $G$ be a linear subspace of $E$ such that $E=F \oplus G$. It is easy to verify that the restriction to $G$ of the canonical mapping $x \mapsto x+F$ is a one-to-one mapping of $G$ onto $E / F$ which preserves the linear space operations. Such a mapping between two linear spaces is called an isomorphism. Thus any linear subspace of $E$ that is complementary to $F$ is "isomorphic" to $E / F$.

Otherwise, let $F$ and $G$ be are linear subspaces of a linear space $E$. The space $E$ is said to be the direct sum of $F$ and $G$ if and only if $E=F+G$ and $F \cap G=\{0\}$. If $E$ is the direct sum of $F$ and $G$ we write $E=F \oplus G$. It is clear that $E=F \oplus G$ if and only if each element $x \in E$ has a unique representation in the form $x=y+z$ where $y \in F$ and $z \in G$. It can be proved that, given a linear subspace $F$ of $E$, there exists at least one linear subspace $G$ of $E$ such that $E=F \oplus G$; any such linear subspace $G$ is said to be complementary to $F$ in $E$.

Theorem 1. Let $F$ be a closed linear subspace of an upper transversal normed space $E$ with the bisection function $g \in \mathfrak{G}([a, b))$ and let

$$
\|x+F\|=\inf \{\|x+y\|: y \in F\}
$$

for all $x \in F$. The mapping $(x+F) \mapsto\|x+F\|$ is an upper transversal norm on $E / F$, and further if $E$ is complete space, so is $E / F$.

The proof of this statement is founded on the facts of problems 13 and 14. A proof for this statement may be found in: Tas kovi ć[2005].

In further, the upper transversal normed spaces $\left(X,\|\cdot\|_{X}\right)$ and $\left(Y,\|\cdot\|_{Y}\right)$ with the same field $\mathbb{K}$ are isometric isomorphism (or isomorphism) if there exists a one-to-one linear transformation (operator) $A: X \rightarrow Y$ such that

$$
\|A x\|_{Y}=\|x\|_{X} \quad \text { for every } \quad x \in X
$$

and $X$ is said to be isometrically isomorphic to $Y$ if there exists an isometric isomorphism of $X$ onto $Y$.

This terminology enables us to give precise meaning to the statement that one upper transversal normed linear space is essentially the same as another. 
In this sense, if the upper transversal normed spaces $X$ and $Y$ are isometrically isomorphic and if one of him complete (upper complete), then other of him also complete space.

Linear upper bounded operators. Let $X$ and $Y$ be upper transversal normed spaces over $\mathbb{K}$ and let $L(X, Y)$ be a set of all linear operators of $X$ into $Y$. An operator $A \in L(X, Y)$ is bounded (or upper bounded) if there exists a real number $M \geq 0$ with property that

$$
\|A x\| \leq M\|x\| \quad \text { for every } \quad x \in X ;
$$

in this case, then $M$ is called a bound (or upper bound) for $A$, and such an $A$ is often referred to as a bounded linear operator, where an upper norm of operator $A$, in notation $\|A\|$, defined by

$$
\|A\|:=\sup _{x \in X \backslash\{0\}} \frac{\|A x\|}{\|x\|}
$$

or on a second manner, as infimum of the numbers $M \geq 0$ for which holds (9). Otherwise, the set $S \subset X$ is bounded (or upper bounded) if there exists $m \geq 0$ such that $\|x\| \leq m$ for every $x \in S$.

In this sense, if $A: X \rightarrow Y$ is a homogeneous operator then the following equalities hold in the form as

$$
\begin{gathered}
\|A\|:=\sup _{\|x\| \leq 1}\|A x\|=\sup _{\|x\|=1}\|A x\|= \\
=\sup _{X \backslash\{0\}} \frac{\|A x\|}{\|x\|}=\min \left\{M \in \mathbb{R}_{+}^{0}:\|A x\| \leq M\|x\| \quad \text { for every } \quad x \in X\right\},
\end{gathered}
$$

where $\min \varnothing=+\infty$; and thus we can obtain and other formulas for $\|A\|$. Specially, for some $M \in \mathbb{R}_{+}^{0}$ holds $\|A\| \leq M$ if and only if $\|A x\| \leq M\|x\|$ for every $x \in X$. Set $M=\|A\|$ then it follows that $\|A x\| \leq\|A\|\|x\|$ for every $x \in X(\infty \cdot 0=0)$.

Illustrations. The identity mapping of $X$ into itself $\operatorname{id}_{X}: X \rightarrow X$ defined by $\operatorname{id}_{X}(x)=x$ for $x \in X$ is linear. Since $\left\|\operatorname{id}_{X}(x)\right\|=\|x\|$ for every $x \in X$, it follows $\left\|\operatorname{id}_{X}\right\|=1$.

If $X$ is a subspace of the space $Y$, then the mapping $A_{X}: X \rightarrow Y$ defined by $A_{X}(x)=x$ for $x \in X$ is linear and $\left\|A_{X}\right\|=1$ (for $X \neq\{0\}$ ).

Amo the rest, if the operator $A: X \rightarrow Y$ is a homogeneous operator, then for it upper norm the following facts hold in the next as

$$
\|A\|=\sup _{x \in X \backslash\{0\}} \frac{\|A x\|}{\|x\|}=\sup _{x \in X \backslash\{0\}}\left\|\frac{1}{\|x\|} A x\right\|=\inf _{x \in X \backslash\{0\}}\left\|A\left(\frac{x}{\|x\|}\right)\right\|,
$$

hence, because of the fact that for every $x \in X \backslash\{0\}$ the upper norm of the vector $x /\|x\|$ is equaly 1 , it follows fact that $\|A\|=\sup _{\|x\|=1}\|A x\|$, which is one of the equalities in (11). Since

$$
\sup _{\|x\|=1}\|A x\|=\|A\| \leq \sup _{\|x\| \leq 1}\|A x\|=\|A\|,
$$


we obtain that the first equality in (11) holds. This with (12) give a good reason for the preceding equalities in (11).

In further, the set of all linear bounded operators of $X$ into $Y$ denoted by $B(X, Y)$; also, the space $B(X, \mathbb{K})$ is denoted by $X^{*}$ and is called the conjugate or more briefly, functionals.

Proposition 4. Let $X$ and $Y$ be two upper transversal normed spaces over $\mathbb{K}$ with the bisection function $g \in \mathfrak{G}([a, b))$. Then $B(X, Y)$ is a vector subspace in $L(X, Y)$ and upper norm of operators is an upper norm on the space $B(X, Y)$.

The proof of this statement is a total analogy with the classic proof of corresponding statement for normed spaces. A proof for this statement may be found in: Taskovi ć[2005].

Proposition 5. Let $X$ be an upper transversal normed space with the upper bisection function $g \in \mathfrak{G}([a, b))$ and let $Y$ be a somplete upper transversal normed space. Then $B(X, Y)$ is a complete upper transversal normed space.

We notice that, in this case, also the proof of this statement is a total analogy with the classic proof of the corresponding statement for normed space $X$ and Banach space $Y$. Thus this proof we omited.

As a direct consequence of Proposition 5 is the following fact that every upper transversal normed space $X$ with the bisection function $g \in \mathfrak{G}([a, b))$ has the complete conjugate space $X^{*}$.

Proposition 6. Let $X$ and $Y$ be two upper transversal normed spaces and $A: X \rightarrow Y$ a linear operator. Then the following conditions are all equivalent to one another: 1) $A$ is upper continuous, 2) $A$ is upper continuous at the origin, 3) $A$ is upper bounded, and 4) if $g(S(0,1)):=\{x:\|x\| \leq 1\}$ is closed unit sphere in $X$, then its image $A(g(S(0,1)))$ is an upper bounded set in $Y$.

Remarks. We notice that if an additive operator is continuous in a point of space, then it is continuous on the entire space. For an additive operator $A: X \rightarrow Y$ the following two facts hold: $A(0)=0$, and $A(-x)=-A(x)$ for every $x \in X$.

Proof of Proposition 6. First, the condition of upper uniformly continuous implies upper continuous (i.e., continuous) of the mapping $A: X \rightarrow Y$ in the point $0 \in X$. On the other hand, if $A \in B(X, Y)$, then for $\delta=\varepsilon / M$ and $M, \varepsilon>0$ from the inequality $\|x-y\|<\delta$ it follows that

$$
\|A x-A y\| \leq M\|x-y\|<\varepsilon,
$$

i.e., this means that $A$ is upper uniformly continuous on the space $X$. Reverse, if $A$ is upper continuous in $0 \in X$, then $A \in B(X, Y)$.

Indeed, if $A$ is upper continuous in $0 \in X$, then for $\varepsilon=1$ there exists $\delta>0$ such that $\|A x\|<1$ whenever $\|x\|<\delta$. Hence, for $x \in X(x \neq 0)$ and 
$0<\delta<\alpha$ it follows that $\|\alpha x /\| x\|\|<\delta$ and that

$$
\|A x\|=\left\|\frac{\|x\|}{\alpha} A\left(\frac{\alpha x}{\|x\|}\right)\right\| \leq \frac{1}{\alpha}\|x\| ;
$$

and thus, since this inequality holds and for $x=0$, it follows that $A \in$ $B(X, Y)$. This means from all the preceding facts that 2 ) is equivalent to $3)$.

On the other hand, since a nonempty subset of an upper normed linear space is upper bounded iff it is contained in a closed sphere centered on the origin, it is evident that 3) implies 4); for if $\|x\| \leq 1$, then $\|A x\| \leq M$. To show that 4) implies 3), we assume that $A(g(S(0,1)))$ is contained in a closed sphere of radius $M$ centered on the origin. If $x=0$, then $A(x)=0$, and clearly $\|A x\| \leq M\|x\|$; and if $x \neq 0$, then $x /\|x\| \in g(S(0,1)))$, and therefore $\|A(x /\|x\|)\| \leq M$, so again we have $\|A x\| \leq M\|x\|$. The proof is complete.

Infinite series in upper normed spaces. In an upper transversal normed space we can introduce the notion of an infinite series. Let $E$ be an upper transversal normed space. A pair of sequences $\left\{x_{n}\right\}_{n \in \mathbb{N}}$ and $\left\{\sigma_{n}\right\}_{n \in \mathbb{N}}$ in $E$ such that $\sigma_{n}=x_{1}+x_{2}+\cdots+x_{n}$ for $n \in \mathbb{N}$ is called an infinite series (or series) and denoted by $\sum_{n \in \mathbb{N}} x_{n}$. The point $\sigma_{n}$ is called the $n$th partial sum of this series.

The series $\sum_{n \in \mathbb{N}} x_{n}$ is said to upper converge (or converge) iff the sequence of partial sums $\left\{\sigma_{n}\right\}_{n \in \mathbb{N}}$ upper converge (converge). An infinite series $\sum_{n \in \mathbb{N}} x_{n}$ in an upper transversal normed space $E$ is said to be absolutely convergent iff the series of real numbers $\sum_{n \in \mathbb{N}}\left\|x_{n}\right\|$ is upper convergent (convergent).

Clearly the absolutely convergent series in the upper transversal normed space $\mathbb{R}$ are just the absolutely convergent series of real numbers in the usual sense of elementary analysis. For a result which generalizes a well-known property of absolutely convergent series of real numbers and for a rearrangement (or commutative convergence) of a series see: Tasković [2005]. Many properties of series of real numbers generalize to series in upper transversal normed space as and in the next statement.

Proposition 7. An upper transversal normed space $X$ with a bisection function $g \in \mathfrak{G}\left(\mathbb{R}_{+}^{0}\right)$ is complete if and only if inside them every absolutely convergent series is convergent.

The proof of this statement is very analogy with the corresponding proof in the case of clssic normed spaces. Thus the proof we omit.

Annotation. We notice that the completeness of upper transversal normed spaces we can describe and via, among the rest, Cauchy's net. ${ }^{2}$ In this

\footnotetext{
${ }^{2}$ Generalized convergence. An ordered set $P:=(P, \preccurlyeq)$ is directed if for each pair $x, y \in P$ there is some $z \in P$ such that $x \preccurlyeq z$ and $y \preccurlyeq z$. A net $\left\{x_{i}\right\}_{i \in P}$ in a set $X$ is any
} 
sense, the net $\left\{x_{i}\right\}_{i \in I}$ in upper transversal normed space $X$ is a Cauchy's net if for every $\varepsilon>0$ there exists $i_{0}=i_{0}(\varepsilon) \in I$ such that $i, j \geq i_{0}$ implies $\left\|x_{i}-x_{j}\right\|<\varepsilon$.

Proposition 8. In every complete upper transversal normed space $X$ with a bisection function $g \in \mathfrak{G}\left(\mathbb{R}_{+}^{0}\right)$ every Cauchy's net is a convergent net.

This statement means that completeness of upper transversal normed spaces we can describe via Cauchy's nets. The proof of this statement is an analogy with the proof of the preceding statement.

Separability. The set $M$ is called dense in $X$ iff $\mathrm{Cl}(M)=X$. The topological space $X$ is called separable iff there is an at most countable set which is dense in $X$.

A nonempty subset $M$ of an upper transversal normed space $E$ is said to be fundamental in $E$ iff the linear hull of $M$ is dense in $E$.

It follows directly from former facts that $M$ is fundamental in $E$ if and only if the closed linear hull of $M$ is $E$.

Proposition 9. An upper transversal normed space $E$ with a bisection function $g \in \mathfrak{G}\left(\mathbb{R}_{+}^{0}\right)$ is separable if and only if there exists a countable subset of $E$ that is fundamental in $E$.

The proof of this statement is a totally analogy with the proof in the case of classical normed spaces. Thus the proof we omit.

Locally compactness. A topological space $X$ is locally compact if every point $x \in X$ has a compact neighborhood. Any discrete space is locally compact, but not compact unless it is finite. The real line $\mathbb{R}$ is locally compact but not compact.

Proposition 10. An upper transversal normed space $X$ is locally compact if and only if the following closed ball in the form

$$
g(K[0,1]):=\{x \in X:\|x\| \leq 1\}
$$

is a compact set. (This fact is characteristic and for classical normed spaces.) Proof. (Necessity). Let $g(K[0,1])$ be a compact set. For arbitrary point $x_{0} \in X$ the mapping $x \mapsto x+x_{0}$ is a homeomorphism of $X$ into $X$, the sets $x_{0}+g(K[0,1])$ and $g(K[0,1])$ are homeomorphic, and thus $x_{0}+g(K[0,1])$ is a compact neighborhood of $x_{0}$.

Sufficiency. Let $X$ be a compact upper transversal normed space, thus the point $0 \in X$ has a compact neighborhood $V$ which is a closed set such that contained a ball $V(0, r)$ for $r>0$. The set

$$
g(K[0, r])=\{x \in X:\|x\| \leq r\}=\mathrm{Cl}(V(0, r)) \subset \mathrm{Cl}(V)=V,
$$

function $x: P \rightarrow X$. If $X$ is a topological space, then the net $\left\{x_{i}\right\}_{i \in P}$ converges to a point $x \in X$ provided for each nbd $U$ of $x$ there is $i_{0}=i_{0}(U) \in P$ such that for all $i \geq i_{0}$ we have $x_{i} \in U$. 
is a compact set (as a compact subset of a compact set $V$ ). This means that the closed ball $g(K[0,1])$ is a picture of the set $g(K[0, r])$ with the continuous mapping $x \mapsto(1 / r) x$, and thus $g(K[0,1])$ is a compact set. The proof is complete.

Corollary 1. The space $\mathbb{R}^{n}$, as an upper transversal normed space, is locally compact. (In this case a closed unit ball is in the form $g(K[0,1])=\{x \in$ $\left.\mathbb{R}^{n}:\|x\| \leq 1\right\}$ is a compact set.)

The forms of Riesz's lemma. From the preceding facts we obtain that a nonempty bounded closed subset of a finite dimensional upper transversal normed space is compact. There is a converse of this fact which is true and which provides an important characterization of finite dimensional upper transversal normed spaces. We shall need the following result.

Proposition 11. (Form of Riesz's lemma). Let $Y$ be a closed proper subspace in an upper transversal normed space $X$. Then for every $\delta \in(0,1)$ there exists a point $x_{\delta} \in X$ such that

$$
\left\|x_{\delta}\right\|=1 \quad \text { and } \quad \rho\left[x_{\delta}, Y\right]:=\inf _{y \in Y}\left\|x_{\delta}-y\right\|>\delta .
$$

The proof of this statement is a totally analogy with the corresponding proof for the case of classical normed space due to F. Riesz in 1918. The following fact is a consequence of the preceding result.

Proposition 12. An upper transversal normed space $X$ with a bisection function $g \in \mathfrak{G}\left(\mathbb{R}_{+}^{0}\right)$ is finite dimensional if and only if every his bounded closed subset is compact.

Otherwise, the following geometrical fact holds on upper transversal normed spaces, which has key role in lower transversal normed spaces.

Proposition 13. Let $Y$ be a closed proper subspace in an upper transversal normed space $X$. Then for every $\delta>1$ there exists a point $x_{\delta} \in X$ such that

$$
\left\|x_{\delta}\right\|=1 \quad \text { and } \quad \operatorname{pdiam}\left(x_{\delta}, Y\right):=\sup _{y \in Y}\left\|x_{\delta}-y\right\|<\delta .
$$

Since this statement also holds in the lower transversal normed spaces, thus his the proof we give only in this case after in the next paragraph.

The Approximation Problem. Let $L$ be an upper transversal normed space and suppose $K \subset L$. For an arbitrary $z \in L$, find an element $z_{0} \in K$, called a best approximation to $z \in K$ such that

$$
\operatorname{dist}(z, K):=\inf _{y \in K}\|y-z\|=\left\|z_{0}-z\right\| .
$$

Two questions are to be considered: 1) Does $K$ contain a best approximation to $z$ ? and 2) If a best approximation exists, is it unique?

The approximation problem is now seen as a problem in minimizing a translate of the upper norm function, or if $z=0$, as problem in minimizing the upper norm function itself. 
The space is $C[0,1]$ the space of continuous functions on $[0,1]$, and $K$ is the subset of all affine functions $x \mapsto m x+b$. The point $z$ to be approximated is $f(x)=x /(x+1)$ and we can consider the problem for each of the upper transversal norms.

Before obtaining any formal results, a number of geometric observations may be helpful. The set $M_{K}(z)$ of best approximations to $z \in K$ is always convex if $K$ is convex, since it is the intersection of the closed ball $N_{\rho}(z)$ of radius $\rho=\operatorname{dist}(z, K)$ with the set $K$. Being convex, $M_{K}(z)$ is either empty, a single point, or an infinite set (as on Figure 2).

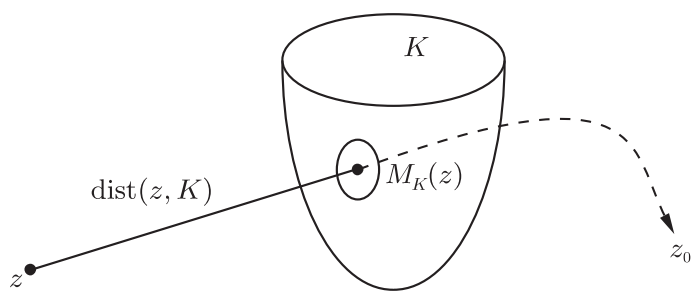

FIGURE 2

As a further guide to our intuition, consider the situation in the plane. Denoting points by $x=(r, s)$ to avoid subscripts, we defined three different upper norms in the following forms as

$$
\|x\|_{1}=|r|+|s|, \quad\|x\|_{2}=\max \{|r|,|s|\}, \quad\|x\|_{3}=\left(r^{2}+s^{2}\right)^{1 / 2} .
$$

They are, except for the dimension of the space, exactly the upper norms that came up in a natural way. Let $K=\{(r, s): r+s \geq 2\}$ and take $z=0$ as the point to be approximated. Imagine a ball about the origin with variable radius $\rho$. Let $\rho$ increase from 0 until the expanding ball approaches the set $K$.

The two questions associated with the approximation problem become the following: First, is there a ball that contains points of $K$ on its boundary $\|x\|=\rho$ but no points of $K$ in its interior $\|x\|<\rho$ ?; and second, if such a ball exists, does is contain just one point of $K$ ? The situation for each of the three upper norms is indicated in Fig. 3.

The distance from $K$ to the origin are, respectively: $2,1, \sqrt{2}$. In all three cases, the ansver to first question is yes. But for second question, it is no for the first, yes for the other two. If the set $K$ were the open half-space, then first question would have a negative answer in all three cases.

Some reflection makes it clear that answers to the approximation questions depend both on properties of the set $K$ (=closed?, compact?, convex?, general convex?, subspace?) and on the geometry of the unit sphere.

Proposition 14. Let $K$ be a finite dimensional closed subset in an upper transversal normed space $L$ with a bisection function $g \in \mathfrak{G}\left(\mathbb{R}_{+}^{0}\right)$. Then there is at least one point of $K$ at a minimum transversal from a given point $z \in L$. 


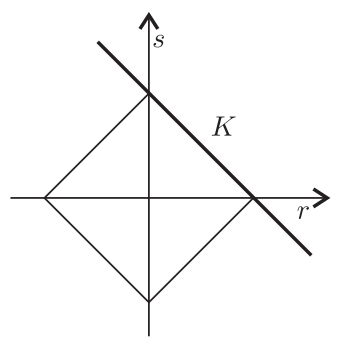

$|r|+|s|=2$

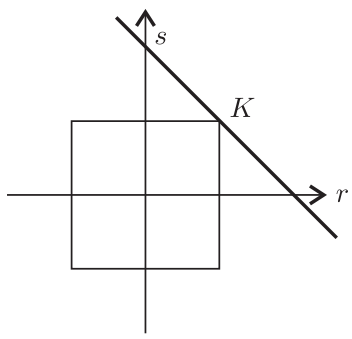

$\max \{|r|,|s|\}=1$

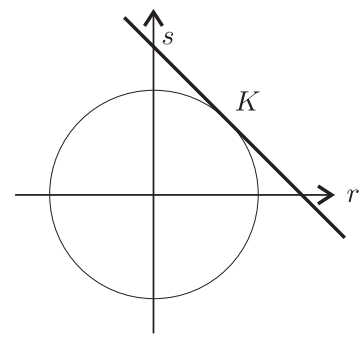

$\left(r^{2}+s^{2}\right)^{1 / 2}=\sqrt{2}$

FiguRE 3

Proof. There is no loss in generality if we take $z=0$. Choose any point $x_{0} \in K$ and form the set $M=\left\{x \in K:\|x\| \leq\left\|x_{0}\right\|\right\}$. The set $M$ is closed and bounded in a finite dimensional subspace of $L$, and thus it is compact. The continuous upper norm function $f(x)=\|x\|$ takes a minimum value at some $z_{0} \in M$, and for any $x \in K$, we have $f(x) \geq f\left(z_{0}\right)$. The proof is complete.

The question of uniqueness remains to be invstigated. Toward this end, not suprisingly, we must define a concept for upper norms that is really a condition that prevents flat spots on the unit sphere. We say an upper transversal normed space $L$ is a strictly convex space if its unit sphere contains no line segments. More precisely, we require that

$$
\|x\|=\|y\|=\left\|2^{-1}(x+y)\right\|=1 \quad \text { implies } \quad x=y .
$$

All inner product spaces (hence all Euclidean spaces $\mathbb{R}^{m}$ ) are strictly convex. We emphasize that strict convexity is a property not preserved by topological isomorphism, as is apparent from considering the (topologically equivalent) upper norms (represented in Fig. 3.).

Proposition 15. Let $K$ be a finite dimensional closed convex subset in a strictly convex upper transversal normed space $L$ with a bisection function $g \in \mathfrak{G}\left(\mathbb{R}_{+}^{0}\right)$. Then there is a unique point of $K$ at a minimum transversal from a given point $z \in L$.

Proof. The existence of a point of $K$ at a minimum transversal from $z$ is guaranteed by Proposition 14. Only the uniqueness needs to be established. Assume as ussual that $z$ is the origin, and let $x$ and $y$ be two points of $K$ at a minimum transversal $\rho>0$ from 0 . Since $K$ is convex we have $2^{-1}(x+y) \in K$ and thus

$$
\rho \leq\left\|2^{-1}(x+y)\right\| \leq 2^{-1}(\|x\|+\|y\|)=\rho\left(2^{-1}+2^{-1}\right)=\rho ;
$$

and thus, $\left\|2^{-1}(x+y)\right\|=\rho$ and so $x / \rho, y / \rho$ and $2^{-1}(x+y) / \rho$ all have upper norm 1. It follows from the strict convexity that $x / \rho=y / \rho$, hence that $x=y$. The proof is complete. 
As an illustration of the use of compactness in analysis we shall establish some important properties of finite-dimensional upper transversal normed spaces, and obtain some characteristic facts of such spaces in terms of compactness. In this sense first is an important fact well known as Riesz's lemma in the following form.

Proposition 16. (Riesz, [1918]). Let $Y$ be a closed proper subspace in a normed space $X$. Then for every $\delta \in(0,1)$ there exists a point $x_{\delta} \in X$ such that

$$
\rho\left(x_{\delta}, Y\right):=\inf _{y \in Y}\left\|x_{\delta}-y\right\|>\delta \quad \text { and } \quad\left\|x_{\delta}\right\|=1 .
$$

If $Y$ is a finite dimensional subspace of $X$, then there exists a unit vector $x_{\delta}$ such that the following equality holds in the form $\rho\left(x_{\delta}, Y\right)=1$.

We notice that if $X$ three-dimensional Euclidean space and if $Y$ is plane, then for $x_{\delta}$ we can to take unit vector $e$ which is perpendicular on the suspace $Y$. Then, from Pythagora's theorem, for every $y \in Y$ the following fact holds

$$
\|e-y\|^{2}=\|e\|^{2}+\|y\|^{2}=1+\|y\|^{2} \geq 1>\delta .
$$

On the other hand, we notice that, any two $n$-dimensional upper transversal normed spaces over the same field with a bisection function $g \in \mathfrak{G}\left(\mathbb{R}_{+}^{0}\right)$ are linearly homeomorphic.

Proof of Proposition 16. From the former observations and from the conditions of this statement, directly it follows that there exists a point $x_{0} \in X \backslash Y$ such that

$$
\rho\left(x_{0}, Y\right)=\inf _{y \in Y}\left\|x_{0}-y\right\|:=\rho>0 .
$$

Since always $\rho<\rho / \delta$, thus it follows that there is a point $z \in Y$ such that $\rho \leq\left\|x_{0}-z\right\|<\rho / \delta$. If choice $x_{\delta}=\left(x_{0}-z\right) /\left\|x_{0}-z\right\|$, then $\left\|x_{\delta}\right\|=1$ and for $y \in Y$ we have

$$
\left\|x_{\delta}-y\right\|=\frac{\left\|x_{0}-\left(z+y\left\|x_{0}-z\right\|\right)\right\|}{\left\|x_{0}-z\right\|} \geq \frac{\rho}{\left\|x_{0}-z\right\|}>\delta,
$$

i.e. (13) holds. For second part of this statement, if $Y$ is finite dimensional, then the subspace $Y_{0}=\operatorname{Lin}\left(x_{0}, Y\right)$ is finite dimensional. Applying the first part of statement on $Y_{0}$, then for every $n \in \mathbb{N}$ there exists unit vector $x_{n} \in Y_{0}$ such that $\rho\left(x_{n}, Y\right) \geq 1 / n$.

The sequence $\left\{x_{n}\right\}_{n \in \mathbb{N}}$ is a sequence of unit vectors in $Y_{0}$ has a convergent subsequence of the form $\left\{x_{p(n)}\right\}_{n \in \mathbb{N}}$ with $\tilde{x}$ as limit. Then $\tilde{x}$ is unit vector and for every $y \in Y$ we obtain $1-1 / p(n) \leq\left\|x_{p(n)}-y\right\|$. Thus as $n \rightarrow \infty$ it follows $1 \leq\|\tilde{x}-y\|$. Since $\|\tilde{x}-y\| \geq 1$ for every $y \in Y$ directly we obtain $\rho(\tilde{x}, Y) \geq 1$.

On the other hand, 0 in $Y$ implies that the following inequality in the form $\rho(\tilde{x}, Y) \leq\|\tilde{x}-0\|=1$. Hence, in final, $\rho(\tilde{x}, Y)=1$. The proof is complete. 
Proposition 17. (Riesz, [1918]). A normed space $X$ is finite dimensional if and only if every its bounded closed subset is compact.

A proof of this statement may be found in: R i es z [1918]. Also, the proof of Proposition 16 is essentially due to F. Riesz in 1918. The preceding result is an important characterization of finite dimensional normed spaces.

Three main theorems on upper normed spaces. The initial combination of algebraic and transversal structures opens up the possibility of studying linear transformations of one upper transversal normed space into another which have the additional property of being continuous.

Most of our work in this chapter of the paper centers around three fundamental theorems relating to continuous linear transformations: Form of Hahn-Banach theorem, Form of open mapping theorem, and Form of uniform boundedness theorem (=Form of Banach - Steinhaus theorem).

The form of Hahn-Banach theorem guarantees that a complete upper transversal normed space is richly supplied with continuous linear functionals, and makes possible an adequate theory of conjugate transversal upper normed spaces.

We turn now to the question of the existence of non-zero bounded linear functionals on an arbitrary non-zero upper transversal normed space. Such functionals obviously exist when the space is one-dimensional, and every non-zero linear space has one-dimensional linear subspaces. Thus, if we can show that any bounded linear functional on a non-zero linear subspace of an upper transversal normed space can be extended to a bounded linear functional defined on the whole space, then it will follow that non-zero bounded linear functionals exist in abundance.

Further, by the "Axiom of Infinte Choice" we mean a statement in the following form as: Given any set $S$, there exist at least countable choice functions or there exist at least finite choice functions.

In general, equivalents of the Axiom of Infinite Choice appear frequently in almost all branches of mathematics in a large variety of different forms.

In this part of the paper we present an equivalent form of the Axiom of Infinite Choice which is expressible in the following sense.

Lemma A. (Lemma of Infinite Maximality, Tasković [2012]). Let $P$ be an inductive partially ordered set with ordering $\preccurlyeq$, then $P$ has at least countable maximal elements or $P$ has at least finite maximal elements.

First, in this part we derive a general extension statement, called the Form of Hahn-Banach theorem, for linear functionals on an arbitrary linear space.

Theorem 2. (Form of Hahn-Banach theorem). ${ }^{3}$ Let $X$ be a linear space over $\mathbb{R}, S$ a subspace in $X$, and $f: X \rightarrow \mathbb{R}$ be an upper transversal norm.

\footnotetext{
${ }^{3} \mathrm{~S}$ t e f a n B a n a ch (1892-1945) - Polish mathematician, born in Cracovieu, great thinker, one of the greatest mathematical talents of the 20th century considered by many
} 
If $h$ is a linear functional on $S$ such that

$$
h(s) \leq f(s) \text { for every } s \in S,
$$

then there exist at least countable or finite functionals $H_{k}: X \rightarrow \mathbb{R}$ such that the following inequality and equality hold in the form as

$$
H_{k}(x) \leq f(x) \text { for all } x \in X
$$

and

$$
H_{k}(s)=h(s) \quad \text { for all } s \in S .
$$

A proof of a variant of this statement can be as the proof in further next of Theorem 6 . Theorem 2 is only a special case of Theorem 6 . In this sense a direct application of this statement is in the following.

Proof of Theorem 2. Applying Theorem 6 (to put that the functional $f$ : $X \rightarrow \mathbb{R}$, de facto, $f(x)=\|x\|$, i.e., that $f$ is an upper transversal norm) we obtain directly Theorem 2 , with all adequate facts.

We notice that the form of open mapping theorem enables us to give a satisfactory description of the projections on a complete upper transversal normed space, and has the important closed graph theorem as one of its consequences. Otherwise, the open mapping theorem was obtained by $\mathrm{Stefan} \mathrm{B} \mathrm{a} \mathrm{n} \mathrm{a} \mathrm{ch} \mathrm{in} 1929$ for the case of classical complete normed spaces, till a general form of this statement was obtained by Juliusz Schauder in 1930.

Theorem 3. (Form of open mapping theorem). Let $X$ and $Y$ be two complete upper transversal normed spaces with a bisection function $g \in \mathfrak{G}\left(\mathbb{R}_{+}^{0}\right)$. If $A$ is a bounded linear operator of $X$ onto $Y$, then $A$ is an open mapping, i.e., $A(G)$ is an open subset in $Y$ for every open subset $G$ in $X$.

Most of the applications of the open mapping theorem depend more directly on the following special case, which we state separately for the sake of emphasis.

as founder of the modern functional analysis. Mathematics school of Stefan Banach was famous and made many well-known mathematicians such as: Mazur, Ulam, Orlicz.

Banach with Mazur and Ulam - that was table number one in "Scottish tavern" in Ljvov. Meetings were held there in the time when it was hard to hold for longer or to drink more than Banach. A meeting, lasting 17 hours is famous, in which an important statement concerning Banach's space was proven, but nobody wrote it down nor reproduced since. Learning from this, Banach's wife gave one big notebook to the barman. This notebook, known as "The Scottish Book", consists of around 150 problems. It was printed in the USA without any changes. For more details see the paper of Hugo Steinhaus: Souvenir de Stefan Banach, Colloq. Math., 1 (1948), 74-80.

Among many works, among which cannot be decided which one is more important than the others in the creation of Banach, we decided on the paper on contraction principle (Sur les opérations dans les ensembles abstraits et leur application aux équations intégrales, Thése de doctorat, Fund. Math. 3 (1922), 133-181), that illustrates best the meaning, essence and far-reaching inspiration of Banach's ideas. 
Proposition 18. A one-to-one continuous linear mapping of a complete upper transversal normed space $E$ onto a complete upper transversal normed space $F$ over the same field and a bisection function $g \in \mathfrak{G}\left(\mathbb{R}_{+}^{0}\right)$ is a linear homeomorphism of $E$ onto $F$.

We notice, in particular of this statement, if a one-to-one linear mapping $T$ of a complete upper transversal normed space onto itself is continuous, then its inverse $T^{-1}$ is automatically continuous.

We now turn to the closed form of graph theorem. Let $X$ and $Y$ be complete upper transversal normed spaces with a bisection function $g \in$ $\mathfrak{G}\left(\mathbb{R}_{+}^{0}\right)$. If we define an upper transverse on the product $X \times Y$ by

$$
\rho\left[\left(x_{1}, y_{1}\right),\left(x_{2}, y_{2}\right)\right]=\max \left\{\left\|x_{1}-x_{2}\right\|,\left\|y_{1}-y_{2}\right\|\right\},
$$

then the resulting topology is easily seen to be the same as the product topology, and convergence with respect to this upper transverse is equivalent to coordinatewise convergence. Now let $A$ be a linear operator of $X$ into $Y$. We recall that the graph of $A$ is that subset of $X \times Y$ which consists of all ordered pairs of the form $(x, A(x))$.

Proposition 19. (Form of Closed Graph Theorem). If $X$ and $Y$ are complete upper transversal normed spaces with a bisection function $g \in \mathfrak{G}\left(\mathbb{R}_{+}^{0}\right)$, and if $A$ is a linear operator of $X$ into $Y$, then $A$ is continuous if and only if its graph is closed.

The proof of this statement is a totally analogy with the classical proof of the Closed Graph Theorem for the case of Banach spaces. Thus the proof of this statement we omit.

The techniques used in the preceding applications of well known Baire's theorem are typical of those used in classical applications. From the point of view of functional analysis, the importance of Baire's theorem derives from the fact that it is the basis of the proofs of two fundamental principles of functional analysis: the principle of uniform boundedness and the open mapping theorem. In this sense we have the following result.

In further, let $B(X, Y)$ be denoted the set of all bounded linear transformations of an upper transversal normed space $X$ into an upper transversal normed space $Y$.

Theorem 4. (Form of Banach-Steinhaus theorem). Let $J$ be an index set, $X$ and $Y$ be upper transversal normed spaces with a bisection function $g \in$ $\mathfrak{G}\left(\mathbb{R}_{+}^{0}\right), X$ be an upper complete space and $A_{j} \in B(X, Y)$ for every $j \in J$. Then $\sup _{j \in J}\left\|A_{j} x\right\|<\infty$ for every $x \in X$ if and only if $\sup _{j \in J}\left\|A_{j}\right\|<\infty$.

This statement in the case for a Banach space $X$ and for a normed space $Y$ is well known as the principle of uniform boundedness. This principle first time in 1922 (for bounded linear functionals) is proved by $\mathrm{H} \mathrm{a} \mathrm{h} \mathrm{n}$, afterwards in 1923 (for continuous linear mappings of Banach spaces) is proved by $\mathrm{Hil} \mathrm{d} \mathrm{e} \mathrm{bran} \mathrm{d} \mathrm{t,} \mathrm{and}$ also in 1927 (in a general form) are proved $\mathrm{B}$ a n a ch and $\mathrm{Ste}$ in haus. 
This case of statement is often called the $B$ a $\mathbf{n}$ a c h $-\mathrm{St}$ e i $\mathbf{n} \mathbf{h}$ a $\mathbf{u}$ theorem, and it has several significiant applications to analysis. See, for example, Z y g m u n d [1959, vol. 1, pp. 165-168].

History of the Theorem of Uniform Boundedness. In 1927, S t e f a $\mathrm{n}$ B a n a ch (1892-1945) and Hug o Steinhaus (1887-1972) - using an idea of S t a n is law S a ks (1897-1942) - discovered that the uniform boundedness theorem could be proved by an application of a theorem which R e n é-L o u is B a i r e (1874-1932) has proved in 1899; he had shown that in $\mathbb{R}^{n}$ the intersection of a countable family of dense open subsets is itself dense. This implies that if

$$
f: \mathbb{R}^{n} \rightarrow \mathbb{R}
$$

is a lower semicontinuous function, then any nonempty open subset $U$ of $\mathbb{R}^{n}$ contains a nonempty open subset $V$ such that the following inequality holds in the form as

$$
\sup _{x \in V} f(x)<\infty .
$$

In this sense, these results and their proofs immediately generalize when $\mathbb{R}^{n}$ is replaced by an arbitrary complete metric space.

Extensions of Hahn-Banach theorem. We start with a definition on linear space $X$. Let $A \subset X$ be a set. We say that $A$ is $Q$-radial at a point $a \in A$ iff for every $y \in X(y \neq 0)$ there exists an $\varepsilon=\varepsilon(y)>0$ such that $a+\lambda y \in A$ for every $\lambda \in \mathbb{Q} \cap(0, \varepsilon)$. Also, if $\lambda x+(1-\lambda) y \in A$ for all $x, y \in A$ and arbitrary $\lambda \in \mathbb{Q} \cap[0,1]$, then we say that $A$ is a $Q$-convex set.

Lemma 1. (Tasković, [1993]). Let $X$ be a linear space, let $D \subset X$ be a set $Q$-convex and $Q$-radial at a point $x_{0} \in D$, and let $L \subset X$ be a linear space (over $\mathbb{Q})$ such that $x_{0} \in L$. Let for the function $f: D \rightarrow \mathbb{R}$ there exists $g:(F(D))^{2} \rightarrow \mathbb{R}$ such that

$$
f(\lambda x+(1-\lambda) y) \leq \max \{f(x), f(y), g(f(x), f(y))\}^{4}
$$

for all $x, y \in D$ and for every $\lambda \in \mathbb{Q} \cap[0,1]$. If $z \notin L$, if $Z=\operatorname{Lin}(L \cup\{z\})$, and if $h: L \rightarrow \mathbb{R}$ is a linear functional such that

$$
h(x) \leq f(x) \quad \text { for every } \quad x \in D \cap L,
$$

\footnotetext{
${ }^{4}$ General convex functions. In an earlier paper (Tas k o vi ć: Math. Japonica, 37 (1992), 367-372), introduced the notion of general convex functions. A function $f: D \rightarrow$ $\mathbb{R}$, where $\mathbb{R}$ denotes the real line and $D$ is a convex subset of $\mathbb{R}^{n}$, is said to be general convex iff there is a function $\psi:(f(D))^{2} \rightarrow \mathbb{R}$ such that

(Max)

$$
f(\lambda x+(1-\lambda) y) \leqslant \max \{f(x), f(y), \psi(f(x), f(y))\}
$$

for all $x, y \in D$ and for arbitrary $\lambda \in[0,1]$. We notice that the set of all convex and quasi convex functions can be a proper subset of the set of all general convex functions. We notice that the upper transversal norm $x \mapsto\|x\|$ is a general convex function. The proof is simple.

Historical facts. The recognition of convex functions as a class of functions to be studied is generally traced to $\mathrm{J}$ e n s e n, but as is usually the case, earlier work can be cited that anticipated what was to come. Hölder proved that if $f^{\prime \prime}(x)>0$, then $f$ satisfied what later came to be known as Jensen's inequality. S t o l z proved that if $f$ is
} 
then there exists a linear functional $H: Z \rightarrow \mathbb{R}$ such that the following inequality holds in the form as

$$
H(x) \leq f(x) \quad \text { for every } \quad x \in D \cap Z
$$

as and, $H \mid L=h$, i.e., there exists an extension of linear functional on $Z$ with a subspace $L$.

Proof. For all $x, y \in L$ and for all $\lambda, \mu \in \mathbb{Q} \cap(0, \infty)$ such that $x+\mu z \in D$ and $y-\lambda z \in D$ we obtain from (14) and (15) the following inequalities in the form as

$$
\begin{gathered}
\frac{\lambda}{\lambda+\mu} h(x)+\frac{\mu}{\lambda+\mu} h(y)=h\left(\frac{\lambda}{\lambda+\mu} x+\frac{\mu}{\lambda+\mu} y\right) \leq \\
\leq f\left(\frac{\lambda}{\lambda+\mu} x+\frac{\mu}{\lambda+\mu} y\right)=f\left(\frac{\lambda}{\lambda+\mu}(x+\mu z)+\frac{\mu}{\lambda+\mu}(y-\lambda z)\right) \leq \\
\leq \max \{f(x+\mu z), f(y-\lambda z), g(f(x+\mu z), f(y-\lambda z))\} ;
\end{gathered}
$$

and thus, over directly calculation, we obtain the following correspondent inequality of the form as

$$
\begin{gathered}
\frac{h(x)-f(x+\mu z)}{\mu} \leq \frac{1}{\lambda}\left[\frac{\lambda+\mu}{\mu} \max \{f(x+\mu z), f(y-\lambda z),\right. \\
\left.g(f(x+\mu z), f(y-\lambda z))\}-h(y)-\frac{\lambda}{\mu} f(x+\mu z)\right]:=R ;
\end{gathered}
$$

and defined $U=\{(x, \mu) \in L \times \mathbb{Q}: \mu>0, x+\mu z \in D\}$ and $V=\{(y, \lambda) \in$ $L \times \mathbb{Q}: \lambda>0, y-\lambda z \in D\}$ from the preceding inequality we obtain the following inequality in the form as in the next

$$
N:=\frac{h(x)-f(x+\mu z)}{\mu} \leq \inf _{V} R
$$

which means that $\alpha:=\sup _{U} N \leq \sup _{U} \inf _{V} R:=\beta$.

Since $D$ is $Q$-radial at $x_{0}$, we have $\left(x_{0}, \mu\right) \in U$ for $\mu \in \mathbb{Q} \cap(0, \varepsilon(z))$ and $\left(x_{0}, \lambda\right) \in V$ for $\lambda \in \mathbb{Q} \cap(0, \varepsilon(-z))$. Consequently $U \neq \varnothing$ and $V \neq \varnothing$. Observe also that $\alpha \leq \beta$ implies that $-\infty<\alpha \leq \beta<+\infty$. In particular $[\alpha, \beta] \neq \varnothing$.

Choose any $c \in[\alpha, \beta]$. Every $t \in Z$ may be uniquely written as $t=x+\lambda z$, where $x \in L$ and $\lambda \in \mathbb{Q}$. For such a $t \in Z$ define $H(t)=h(x)-c \lambda$. It is easily seen that $H: Z \rightarrow \mathbb{R}$ is a linear functional and that $H \mid L=h$. Now

continuous on $[a, b]$ and satisfies:

$$
f\left(\frac{x+y}{2}\right) \leq \frac{f(x)+f(y)}{2},
$$

then $f$ has left and right derivatives at each point of $(a, b)$. $\mathrm{H}$ a d a $\mathrm{m}$ a r d obtained a basic integral inequality for functions having an increasing derivative on $[a, b]$. J e $\mathrm{n}$ s e $\mathrm{n}$ used (Je) to define convex functions and gave the first in a long series of results which together with (Je) imply the continuity of $f$. 
take an arbitrary $t \in D \cap Z$ which may be represented in form $t=x+\lambda z$. Consider three cases:

1) If $\lambda=0$, then $t=x$; and by inequality (15) we obtain $H(t)=h(x) \leq$ $f(x)=f(t)$. Consequently this fact we have that inequality (16) holds.

2 ) If $\lambda>0$, then since $t \in D$ we have $(x, \lambda) \in U$. Since $c \geq \alpha$, this inequality implies $h(x)-f(x+\lambda z) \leq c \lambda$, i.e., the following want fact (16) holds in the form as

$$
H(t)=h(x)-c \lambda \leq f(x+\lambda z)=f(t) .
$$

3) If $\lambda<0$, then since $t \in D$ we have $(x,-\lambda) \in V$. Since $c \leq \beta$, this inequality implies that the following inequality holds in the form as

$$
\begin{gathered}
-c \lambda \leq \frac{-\lambda+\mu}{\mu} \max \{f(x+\mu z), f(x+\lambda z), g(f(x+\mu z), f(x+\lambda z))\}- \\
-h(x)+\frac{\lambda}{\mu} f(x+\mu z),
\end{gathered}
$$

and thus, in the context of fact since is defined functional $H(t)$, directly for $\mu=\lambda$ we obtain the following want fact (16) in the form as

$$
H(t)=h(x)-c \lambda \leq f(x+\lambda z)=f(t),
$$

i.e., the inequality (16) holds in this case, too. Now, together with all the preceding facts, the proof is complete.

As an immediate application of Lemma 1 hence we derive the rational version of a form of the Hahn-Banach theorem in the following form as.

Theorem 5. (Rational version extension). Let $X$ be a real linear space, let $D \subset X$ be a set $Q$-convex and $Q$-radial at a point $x_{0} \in D$, and Let $L \subset X$ be a linear space $($ over $\mathbb{Q})$ such that $x_{0} \in L$. If the function $f: D \rightarrow \mathbb{R}$ satisfying (14) and if $h: L \rightarrow \mathbb{R}$ is a linear functional such that (15), then there exist at least countable or finite linear functionals $H_{k}: X \rightarrow \mathbb{R}$ such that

$$
H_{k}(x) \leq f(x) \text { for all } x \in D,
$$

as and, $H_{k} \mid L=h$, i.e., there exist at least countable or finite extensions of linear functionals on $X$ with a subspace $L$.

With regard to the preceding statements extension an immediately general result it follows directly in the following form as.

Theorem 6. (Tasković, [1993]). Let $C$ be a convex open set in upper transversal normed space $X$, let $D$ be a nontrivial subspace such that $D \cap C$ is a nonempty set, and let $f: C \rightarrow \mathbb{R}$ be a general convex function. If $h: D \rightarrow \mathbb{R}$ is an affine mapping such that

$$
h(x) \leq f(x) \quad \text { for every } \quad x \in D \cap C,
$$


then there exist at least countable or finite affine extensions $H_{k}: X \rightarrow \mathbb{R}$ of the functional $h$ such that the following inequality holds in the form as

$$
H_{k}(x) \leq f(x) \quad \text { for every } \quad x \in D .
$$

Proof of Theorem 5. Let $\mathfrak{R}$ be the family of all couples $(Y, A)$, where $Y$ is a linear space (over $\mathbb{Q}$ ), $L \subset Y \subset X$, and $A: Y \rightarrow \mathbb{R}$ is a linear functional such that $A \mid L=h$ and

$$
A(x) \leq f(x) \text { for all } x \in D \cap Y,
$$

where $(L, h) \in \mathfrak{R}$, so $\mathfrak{R}$ is a nonempty family. We introduce the order in $\mathfrak{R}$ in the usual manner: $\left(Y_{1}, A_{1}\right),\left(Y_{2}, A_{2}\right) \in \mathfrak{R}$ we agree that $\left(Y_{1}, A_{1}\right) \preccurlyeq\left(Y_{2}, A_{2}\right)$ if $Y_{1} \subset Y_{2}$ and $A_{2} \mid Y_{1}=A_{1}$. If $Z \subset \Re$ is any chain, then put

$$
G=\bigcup_{(Y, A) \in Z} Y,
$$

and define $B: G \rightarrow \mathbb{R}$ putting $B(y)=A(y)$ if $y \in Y$ and $(Y, A) \in Z$. The couple $(G, B)$ is an upper bound of $Z \in \mathfrak{R}$. In fact, if $x, y \in G$ and $\alpha \in \mathbb{Q}$, then there exists an $(Y, A) \in Z$ such that $x, y \in Y$. Then also $x+y \in Y \subset G$ and $\alpha x \in Y \subset G$, which shows that $G$ is a linear space (over $\mathbb{Q}$ ). Since $L \subset Y \subset X$ for all $Y$ such that $(Y, A) \in Z$, also $L \subset G=\bigcup_{(Y, A) \in Z} Y \subset X$. Similarly it is shown that $B$ is a linear functional fufilling (18) and such that $B \mid L=h$.

By Lemma of Infinite Maximality in $\mathfrak{R}$ there exist at least countable or finite maximal elements $\left(E_{k}, H_{k}\right)$. The only thing we need to show is that $E_{k}=X$. Supposing the contrary, let $z \in X \backslash E_{k}$. By Lemma 1 there exists a linear functional $H_{k}^{*}: \operatorname{Lin}\left(E_{k} \cup\{z\}\right) \rightarrow \mathbb{R}$ such that $H_{k}^{*} \mid E_{k}=H_{k}$, whence $H_{k}^{*}\left|L=H_{k}\right| L=h$, and

$$
H_{k}^{*}(x) \leq f(x) \quad \text { for every } \quad x \in D \cap \operatorname{Lin}\left(E_{k} \cup\{z\}\right),
$$

and consequently this $\left(\operatorname{Lin}\left(E_{k} \cup\{z\}\right), H_{k}^{*}\right) \in \mathfrak{R}$, and clearly $\left(E_{k}, H_{k}\right) \prec$ $\left(\operatorname{Lin}\left(E_{k} \cup\{z\}\right), H_{k}^{*}\right)$, which contradicts maximality of $\left(E_{k}, H_{k}\right)$. Consequently we must have $E_{k}=X$. The proof is complete.

Some annotations. We notice the following interesting fact. Let $X$ be a linear space and let $D \subset X$ be a set which is symmetric with respect to 0 (i.e., $-D=D$ ), and let $f: D \rightarrow \mathbb{R}$ be an even function:

$$
f(-x)=f(x) \text { for all } x \in D,
$$

and further, let $L \subset X$ be a linear space (over $\mathbb{Q}$ ), and let $h: L \rightarrow \mathbb{R}$ be a linear functional fulfilling (15), then the following inequality holds in the form as

$$
\mid h(x) \| \leq f(x) \text { for all } \quad x \in D \cap L .
$$

Indeed, take an arbitrary $x \in D \cap L$. We have by (15) and (19) that $h(-x) \leq$ $f(-x)=f(x)$, whence $h(x)=-h(-x) \geq-f(x)$. This together with (15) yields $-f(x) \leq h(x) \leq f(x)$, which is equivalent to $(20)$. 
With regard to preceding facts, in the special case, if $D \subset \mathbb{R}^{n}$ and if the function $f: D \rightarrow \mathbb{R}$ satisfying the following inequality in the form as

$$
f(\lambda x+(1-\lambda) y) \leq \lambda f(x)+(1-\lambda) f(y)
$$

for all $x, y \in D$ and for arbitrary $\lambda \in \mathbb{Q} \cap[0,1]$ the preceding statement are considered M. E. K u c z m a [1970] and E. B e r z [1975].

In connection with this in the book: Kolmogorov - Fom in [1973] is proved a statement of the form of Hahn - Banach theorem on linear spaces, where $f: D \rightarrow \mathbb{R}$ (for $D \subset \mathbb{R}^{n}$ ) is a convex and homogeneous function.

In the preface of the second edition this book in $1976 \mathrm{Kol}$ m o g o r o v is to set down in writing that is to make a essential change with introduction an extension of Hahn-Banach theorem and further connection with problems of extremal tasks. A very similar statement of this may be found in: R o b e r t s - V a r b e r g [1973].

Also, We s t o n [1960], from Bourbaki suggestion, is proved similar statement with an application of Mazur's separation theorem for convex sets and hyperplanes.

On the other hand, $\mathrm{K} \mathrm{a} \mathrm{k} \mathrm{u} \mathrm{t} \mathrm{a} \mathrm{n} \mathrm{i} \mathrm{in} 1938$ is given a first proof of new proofs of Hahn-Banach theorem via Markoff-Kakutani theorem of fixed point (see: Theorem 4.24). For further hystorical facts see the book of History of Functional Analysis in 1981 by D i e u d o n né. Also see: K ön i g [1970] and S i m o n s [1985].

Otherwise, from the preceding rational version extension of a form of HahnBanach theorem we obtain, among the rest, a general version of this statement in a profitable form for further applications, see: T a s k o v i ć [2005].

Theorem 7. (General Hahn-Banach theorem). Let $X$ be a real linear space, let $D \subset X$ be a subspace, and let $f: X \rightarrow \mathbb{R}$ be a general convex function such that

$$
h(x) \leq f(x) \quad \text { for every } \quad x \in D,
$$

where $h: D \rightarrow \mathbb{R}$ is a linear functional. Then there exist at least countable or finite linear functionals $H_{k}: X \rightarrow \mathbb{R}$ such that the following inequality holds in the form as

$$
H_{k}(x) \leq f(x) \text { for every } x \in X,
$$

as and, $H_{k} \mid D=h$, i.e., there exist at least countable or finite extensions of a linear functional on $X$ with a subspace $D$.

We notice that the upper transversal norm $x \mapsto\|x\|$ is a general convex function. The proof is simple. See chapter 4 of this book!

Lemma 2. Let $X$ be a real linear space and $x_{0} \in X$. If $f: X \rightarrow \mathbb{R}$ is a general convex with tent function, then there exists at least countable or finite linear functionals $H_{k}: X \rightarrow \mathbb{R}$ such that

$$
H_{k}\left(x_{0}\right)=f\left(x_{0}\right) \quad \text { and } \quad H_{k}(x) \leq f(x) \text { for all } \quad x \in X .
$$

Open problem 1. Independent of the preceding General Hahn-Banach theorem to make a new proof of Lemma 2 with an application of MarkoffKakutani theorem of fixed point (Theorem 4.24)!? As a pattern for this problem see: H i r a n o-K o m i y a - Taka has h i [1982]. 
Theorem 8. (Separation of concavity and general convexity). Let $E$ be a real linear space, let $D \subset E$ be a nonempty convex subset, and let $f: E \rightarrow \mathbb{R}$ be a general convex with tent function such that

$$
k(x) \leq f(x) \quad \text { for every } \quad x \in D,
$$

where $k: D \rightarrow \mathbb{R}$ is a concave functional. Then there exist at least countable or finite linear functionals $H_{k}: E \rightarrow \mathbb{R}$ such that

$$
k(x) \leq H_{k}(x) \quad \text { for every } \quad x \in D,
$$

and

$$
H_{k}(y) \leq f(y) \quad \text { for every } y \in E .
$$

A brief proof of this statement may by found in Taskovic [2005]. For the proof of this statement the preceding result of Lemma 3 is essential.

This statement means de facto that an arbitrary concave function can be separated with a linear functional satisfying (18) from a general convex function.

In connection with this we notice that $\mathrm{Hir}$ ano-Komiya- Takah a shi [1982] are proved that a concave function (under correspondent conditions) can be separated from an additive and homogeneous function, i.e., from sublinear function. Their proof is founded on Markoff-Kakutani theorem of fixed point (Theorem $4.24)$.

As an immediate consequence of the preceding result of Theorem 4 we obtain in a practical way the following version of Hahn-Banach theorem, see: Tasković [2005].

Proposition 20. (Form of Hahn-Banach theorem). Let $E$ be a real linear space, let $L \subset E$ be a linear subspace, and let $f: E \rightarrow \mathbb{R}$ be a general convex with tent function such that

$$
h(x) \leq f(x) \text { for all } x \in L,
$$

where $h: L \rightarrow \mathbb{R}$ is a linear functional. Then there exist at least countable or finite linear functionals $H_{k}: E \rightarrow \mathbb{R}$ such that

$$
H_{k}(x)=h(x) \quad \text { for all } \quad x \in L
$$

and

$$
H_{k}(y) \leq f(y) \quad \text { for all } y \in E .
$$

Proof. From Theorem 6 there exists $H_{k} \in E^{*}$ satisfying $H_{k}(x) \geq h(x)$ for all $x \in L$ and $H_{k}(y) \leq f(y)$ for all $y \in E$. Since $L$ is a linear subspace of $E^{*}$, we obtain $H_{k}(x)=h(x)$ for all $x \in L$. The proof is complete.

Let $E$ be a linear functional and let $f: E \rightarrow \mathbb{R}$ be a general convex with tent function. For two nonempty subsets $A$ and $B$ of $E$, we consider a number

$$
f(A, B):=\inf \{f(x-y): x \in A, y \in B\} .
$$


As a directly consequence of the preceding facts and results we obtain the following statement.

Further annotations. From the proof of this statement in: Ta s k o v i ć [2001] we have that the proof is founded on separated of a concave function from a general convex function (see: Theorem 8).

On the other hand, an extension of Hahn-Banach theorem which is very similar to the former statement (for convex and concave envelopes) has been given by Kot arski [1987].

This result is a consequence of fact on separated a concave function from a sublinear function, see: Hir a n o, Kom i ya and Takah a shi [1982].

Otherwise, in special case, if $D=L$ (a subspace in $X$ ), $k=H_{0}: L \rightarrow \mathbb{R}$ is a linear functional, and if $f=p: X \rightarrow \mathbb{R}$ is a sublinear functional, then from Theorem 7 directly it follows a form of Hahn-Banach theorem. Indeed, then there exists a linear functional $H$ such that

$$
H_{0}(x) \leq H(x) \quad \text { for every } \quad x \in L
$$

and that is $H(x) \leq p(x)$ for every $x \in L$. Since $L$ is a linear subspace we obtain $H_{0}(x)=H(x)$ for all $x \in L$. Thus, $H$ is an extension of the functional $H_{0}$.

In connection with the preceding facts, B i t $\mathrm{n}$ e r [1974] is considered a sublinearization of the functional $P: X \rightarrow \mathbb{R}$ defined in the following form as

$$
P(x)=\inf _{\lambda>0} \frac{1}{\lambda}\left(\sup _{\alpha \in I} p_{\alpha}(\lambda x)\right) \quad \text { for } \quad x \in X,
$$

where $p_{\alpha}: D \rightarrow \mathbb{R}(\alpha \in I)$ are convex functionals. Evidently, from definition, we obtain $P(\lambda x)=\lambda P(x)$ for every $x \in X$ and for arbitrary $\lambda \geq 0$. Also, because convexity of correspondence functionals, immediate it follows that $P(x)$ is an additive functional.

From these facts and from further phenomens of convex (concave) envelopes we can to come to yet some results in the preceding context. Also see: K o t k o w s k i M a g d zi ar z-Was zak [1977].

In this part we use a form of Hahn-Banach theorem to establish the existence of non-zero bounded linear functionals on an arbitrary transversal upper normed linear space. The Form of Hahn - Banach theorem is concerned with real linear spaces, so our first task is to extend it to complex linear spaces. In the case of the Hahn-Banach theorem an extension to complex linear spaces was obtained in 1938 by Bohnenblust and Sobczyk and, independently, by Soukhomlinoff.

In the context of the preceding facts, we considered the following extension of Theorem 7 on complex linear spaces, in the following form as.

Theorem 9. Let $X$ be a complex linear space, let $Y \subset X$ be a subspace, and let $f: X \rightarrow \mathbb{R}$ be a general convex homogeneous function such that

$$
|h(y)| \leq f(y) \quad \text { for every } y \in Y,
$$

where $h: Y \rightarrow \mathbb{C}$ is a linear functional. Then there exist at least countable or finite linear functionals $H_{k}: X \rightarrow \mathbb{C}$ such that the following inequality 
holds in the form as

$$
\left|H_{k}(x)\right| \leq f(x) \quad \text { for every } \quad x \in X,
$$

as and, $H_{k}(y)=h(y)$ for all $y \in Y$, i.e., there exist at least countable or finite extensions of linear functionals on $X$ with a subspace $Y$.

Inclosing annotations. We notice that Hahn-Banach theorem is an essential and deep fact of entire mathematics. This extension theorem has many applications and without Hahn-Banach theorem functional analysis and mathematics general to looked strange and by all different.

We give only some of applications of Hahn-Banach theorem: adding of normed (upper) space and normed algebra, Schauder's theorem on compact operators, Riesz's theorem on representation of space $C^{*}[a, b]$, Krein's theorem on extension of positive linear functionals, general Banach's integral - limes, difficulty and readily measure problem, Nagy's theorem on uniform boundedness group of linear operators on Hilbert space, separated of convex sets: and functions, as and many other facts of nonlinear functional annalysis.

Otherwise, Hahn-Banach theorem has an essential role for the proof of fact that there exists a Green's function for Laplace equations as and for other boundary value problems (see: G a r a b e di a n [1950], G a r a b e d i a n - S h i f f m a n [1954], L a x [1952], and M i r a n d a [1947]).

Also, in the physics, via Hahn-Banach theorem it follows that there exists a nontrivial quantum field. In this sense in further see Figure 4.

Open problem 2. Does general Hahn-Banach theorem (Theorem 7) is best extension in the sense as "maximal extension" of the Hahn - Banach theorem? Precisely, can be we to change the general convex function $f(x)$ in $(21)$ and (22) with some function in a widening class of functions such that to remain that holds Theorem $7 ?$ ! Does this is posibly?

With regard to the preceding facts we notice that the following problem is wellknown: If $p: X \rightarrow \mathbb{R}$ ( $X$ is a real linear space) is a sublinear functional (i.e., $p(x+y) \leq p(x)+p(y), p(\lambda x)=\lambda p(x)$ for $\lambda \geq 0$ and $x \in X)$, does there exists a real linear functional $H: X \rightarrow \mathbb{R}$ such that

$$
H(x)<p(x) \text { for every } \quad x \neq 0 ? \text { ! }
$$

A necessary condition for this problem is fact that $p(x)+p(-x) \neq 0$ for every $x \neq 0$. In this sense, A r o n s z a j n [1935] is proved that if $X$ is a separable space via norm defined by $\|x\|=p(x)+p(-x)$, then this condition is and sufficient. B o n s a 11 [1954] is proved that the condition of separability can not to omit!

Axiom of Infinite Choice and Extension Theorems. In this sense, the first development occurred on the frontier between algebra, analysis, and sets theory: Stefan Banach's researches at Lwów on functional analysis. In 1929 Banach established a fundamental result later known as the HahnBanach theorem in the following form: Let $p$ be a real-valued functional on a complete normed vector space $V$ over $\mathbb{R}$ and suppose that for every $x$ and $y$ in $V, p(x+y) \leq p(x)+p(y)$ and that $p(\lambda x)=\lambda p(x)$ for all positive $\lambda$; 


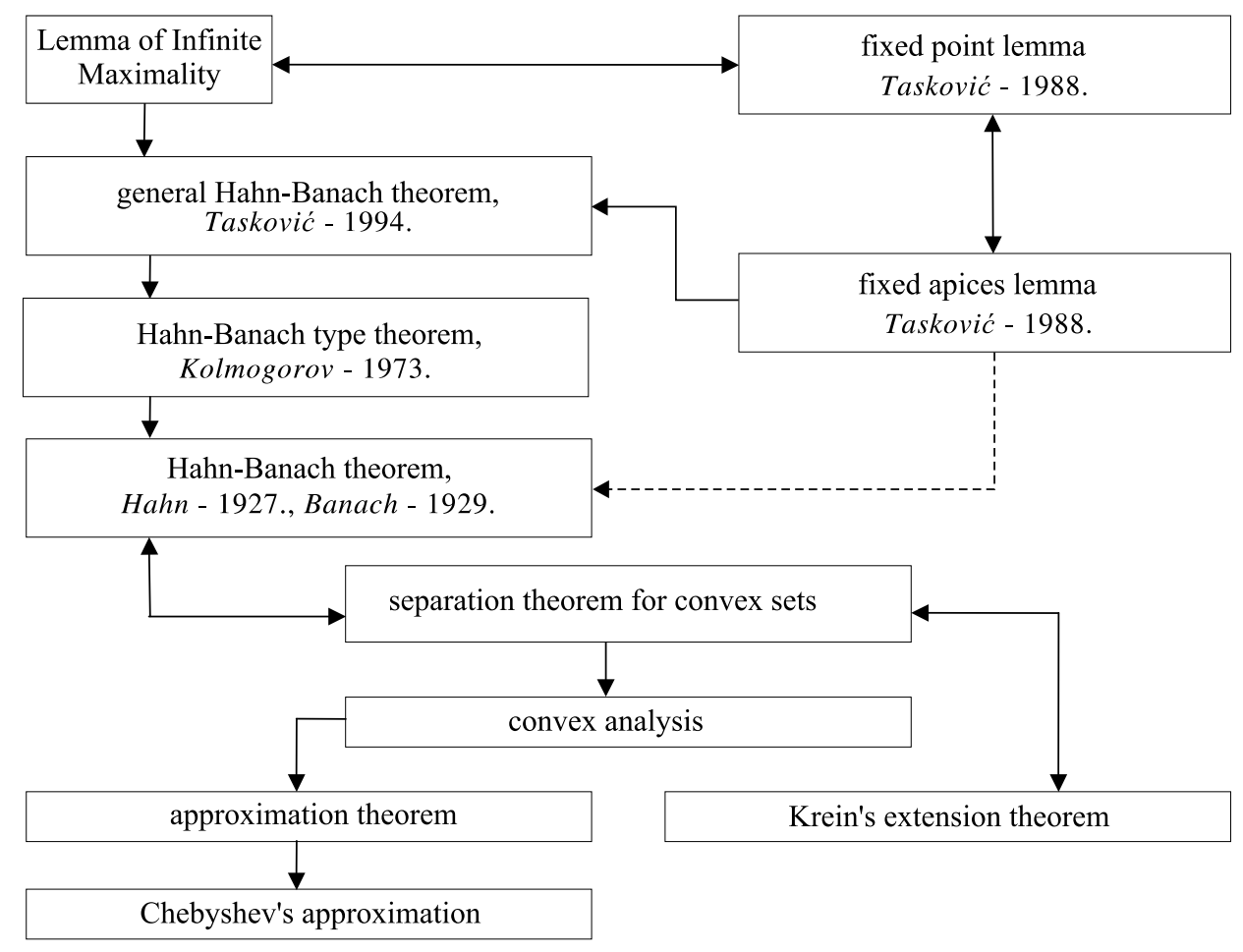

FiguRe 4

then there is an additive functional $f$ such that

$$
-p(-x) \leq f(x) \leq p(x) \text { for every } \quad x \in V .
$$

To obtain this result, Banach relied on the Well-Ordering theorem. Much later, the Hahn-Banach theorem was deduced from the Boolean Prime Ideal theorem and shown to be equivalent to the following sttement: On every Boolean algebra there exists an additive real-valued measure $\mu$ with $\mu(0)=0$ and $\mu(1)=1$.

On the prehistory of the Hahn-Banach theorem, especially as it concerns Eduard Helly and Hans Hahn, see: Hochstadt [1980]. Also see: Łoś and Ryll-Nardzewski [1951], and Luxemburg [1969].

From the preceding facts our general Hahn-Banach theorem is only a consequence of Axiom of Infinite Choice. In the next, we give a variant of Theorem 7 which is an equivalent of Axiom of Infinite Choice.

Theorem 10. (Tasković, [2005]). Let $M$ be a subspace of real linear space $E$ and let $S \subset E$ be a subset. If $f: E \rightarrow \mathbb{R}$ is a general convex functional such that dominated (or $f$-dominated) over linear functional $h: M \rightarrow \mathbb{R}$, 
i.e., such that

$$
h(x) \leq f(x) \text { for every } x \in M,
$$

then the (convex) set $G$ of all $f$-dominated linear extensions $H_{k}: E \rightarrow \mathbb{R}$ have at least countable or finite elements $g_{k}$ such that $g_{k}$ is an extreme point of $G$ and $g_{k}$ is $S_{k}$-maximal in $G$, i.e., for any $\xi \in G$ with $g_{k}(s) \leq \xi(s)$ for every $s \in S$, and $g_{k}(s)=\xi(s)$ for every $s \in S$.

We notice that the special cases of this statement, when the functional $f: E \rightarrow \mathbb{R}$ is sublinear, are proved in different variants: B o n s a ll [1954], A n d e n a es [1970], and L e m b cke [1979].

Proof of Theorem 10 If $S_{k} \subset M$, then the general Hahn-Banach theorem is applicable, so we assume thet $S_{k} \backslash M$ is nonempty. Let $M\left(S_{k}\right)$ denote the subspace of $E$ sppaned by $M$ and $S_{k}$. We now extend $h$ to $M\left(S_{k}\right)$.

Repetition, at the same condition, the procedure in last part of the proof of Theorem 5 we obtain that there exist at least countable or finite extensions linear functional $H_{k}\left(H_{k} \mid M=h, H_{k} \leq f\right)$ such that it a $S_{k}$-maximal element.

Let $\mathfrak{F}$ denotes the set of all linear functionals $H_{k}$ defined on $E$ which are extensions of functional $h$ such that $H_{k} \leq f$.

If we defined an ordereing on the set $\mathfrak{F}$ such that $H_{k} \preccurlyeq S_{k} G$ if and only if $H_{k}(x) \leq G(x)$ for every $x \in S_{k}$, then the preceding fact has a form in the following sense that $\mathfrak{F}$ has at least one element which is maximal via ordering $\preccurlyeq S_{k}$. If $M^{\prime} \supset M$ is a subspace in $E$, let $\mathfrak{F}_{M^{\prime}}=\left\{H_{k} \mid M^{\prime}: H_{k} \in \mathfrak{F}\right\}$. Evidently $\mathfrak{F}_{M^{\prime}}$ is a convex set and there exist $S_{k}$-maximal extensions $H_{k}$ of $h$ such that $H_{k} \mid M\left(S_{k}\right)$ is an extremal point in the set $\mathfrak{F}_{M\left(S_{k}\right)}$.

Let $\mathfrak{R}$ be a family of pairs $\left(h^{\prime}, M^{\prime}\right)$ such that: $(h, M) \preccurlyeq\left(h^{\prime}, M^{\prime}\right), h^{\prime} \leq$ $f \mid M^{\prime}, h^{\prime}$ is maximal on $M^{\prime} \cap S_{k}, M^{\prime}$ is lineal of $M$ and $M^{\prime} \cap S_{k}$, and $h^{\prime}$ is an extremal point in $\mathfrak{F}_{M^{\prime}}$. If $h_{0}$ is an extension of $M_{0}$ to $M_{1}$ defined the linear functional $h_{1}$ on $M_{1}$ with

$$
h_{1}\left(x+\lambda x_{1}\right)=h_{0}(x)+\lambda h_{1}(x)
$$

for every $x \in M_{0}$ and for arbitrary $\lambda \in R$, where $h_{1}\left(x_{1}\right)$ is a value of functional $h_{1}$ in the point $x_{1}$ in the following form as

$$
h_{1}\left(x_{1}\right)=\inf \left\{h_{0}(x)+f\left(x_{1}-x\right): x \in M\right\} .
$$

Hence the preceding properties of $\mathfrak{R}$ hold. To verify only last property. In this sense, assume that $h_{1}=\lambda g+(1-\lambda) r(0<\lambda<1)$, where $g$ and $r$ are linear functionals on $M_{1}$, such that $g|M=r| M=h, g \leq f \mid M_{1}$, and $r \leq f \mid M_{1}$. On the other hand, $h_{1}\left|M_{0}=g\right| M_{0}=r \mid M_{0}$, because $\left(h_{0}, M_{0}\right)$ satisfy the last condition for the family $\mathfrak{R}$.

Hence, $g\left(x_{1}\right) \leq h_{1}\left(x_{1}\right)$ and $r\left(x_{1}\right) \leq h_{1}\left(x_{1}\right)$. Since $h_{1}\left(x_{1}\right)=\lambda g\left(x_{1}\right)+$ $(1-\lambda) r\left(x_{1}\right)$ it follows that $h_{1}\left(x_{1}\right)=g\left(x_{1}\right)=r\left(x_{1}\right)$, which means that is $h_{1}=g=r_{1}$, i.e., $h_{1}$ is an extremal point of $\mathfrak{F}_{M_{1}}$. The proof is complete. 
Annotations. We notice that when applied to Choquet theory the preceding statement will provide extreme boundary measures.

We make some observations which will become useful later. The setting is the same as in Theorem 10 We define $\tilde{f}: E \rightarrow \mathbb{R}$ by

$$
\tilde{f}(x)=\inf \{h(y)+f(x-y): y \in M\},
$$

hence if $S=\left\{x_{0}\right\}$, then for any $S$-maximal extension $H \in \mathfrak{F}$ we have $H\left(x_{0}\right)=$ $\tilde{f}\left(x_{0}\right)$.

Also, for each $x_{0} \in S$ there exists a $S$-maximal extension $H_{0} \in \mathfrak{F}$ such that $H_{0}\left(x_{0}\right)=\tilde{f}\left(x_{0}\right)$ and such that $H_{0} \mid M(S)$ is extreme point in $\mathfrak{F}_{M(S)}$.

A notrivial application. We now show our results can be used to develop some fundamental facts in Choquet theory.

Let $K$ be a nonempty compact convex subset of a locally convex Hausdorff space over the reals. As usual $C(K)$ denotes the class of continuous functions on $K$. An element $\mu \in C(K)^{*}$, with $\mu(1)=1=\|\mu\|$, is called a boundry measure if

$$
\mu\left(K \backslash\left\{x: \sup \left(\mu(f): \mu \in \mathfrak{M}_{x}^{+}\right)=f(x)\right\}\right)=0
$$

for every $f \in C(K)$, where $\mathfrak{M}_{x}^{+}$is a subset of $C(K)^{*}$ consisting of the extensions $\mu$ of a positive linear functional on the set

$$
A=\{f \in C(K): f \quad \text { is an affine function }\} .
$$

In the context of all the preceding facts in Choquet theory the following fact holds that: for each $x \in K$ there exists a boundary measure $\mu \in \mathfrak{M}_{x}^{+}$, see: $\mathrm{C} \mathrm{ho}$ q u e t [1953].

The Figure 5 give all relations of the preceding statements in this part of the book.

Theorem 11. (Sandwich Theorem). Real functions $f, g: I \rightarrow \mathbb{R}(I \subset \mathbb{R}$ is an interval) satisfy $(R)$ for all $x, y \in I$ and arbitrary $\lambda \in[0,1]$ if and only if there exists a general convex function $h: I \rightarrow \mathbb{R}$ for a continuous increasing function $S: h(I)^{2} \rightarrow \mathbb{R}$ such that

$$
f(x) \leq h(x) \leq g(x) \quad \text { for every } \quad x \in I .
$$

Arguing as in the proof of Theorem 11, by Tasković [2001], we can get however the following result.

Theorem 12. (Tasković, [2001]). Real functions $f, g: D \rightarrow \mathbb{R}(D$ is a convex subset of a vector space) satisfy for a continuous increasing function $G: g(D)^{n} \rightarrow \mathbb{R}$ the following inequality

$$
f\left(\sum_{j=1}^{n} \lambda_{j} x_{j}\right) \leq \max \left\{g\left(x_{1}\right), \ldots, g\left(x_{n}\right), G\left(g\left(x_{1}\right), \ldots, g\left(x_{n}\right)\right)\right\}
$$

for all $n \in \mathbb{N}$, for all $x_{1}, \ldots, x_{n} \in D$ and for reals $\lambda_{1}, \ldots, \lambda_{n} \in[0,1]$ with the property $\lambda_{1}+\cdots+\lambda_{n}=1$ if and only if there exists a general convex 


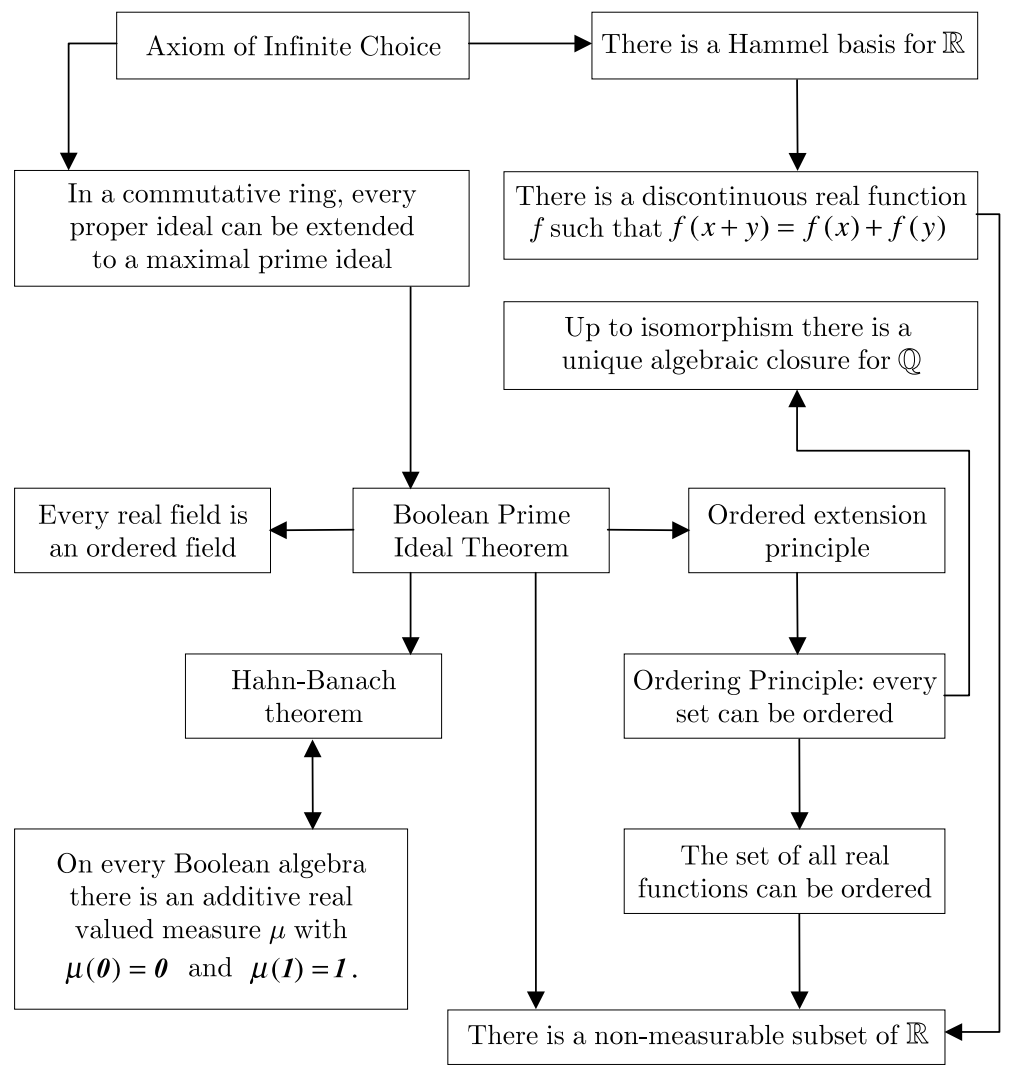

FigURE 5

function $h: D \rightarrow \mathbb{R}$ for a continuous increasing function $S: h(D)^{n} \rightarrow \mathbb{R}$ such that

$$
f(x) \leq h(x) \leq g(x) \quad \text { for every } \quad x \in D .
$$

We notice, that real functions $f$ and $g$, defined on a convex subset $D$ of an $(n-1)$-dimensional real linear space, satisfy $(23)$ if and only if there exists a general convex function $h: D \rightarrow \mathbb{R}$ satisfying (24).

We notice that the following special case of Theorem 12 is very constructive in this context. Namely, for the functional inequality of the form as

$$
f(\lambda x+(1-\lambda) y) \leq \lambda g(x)+(1-\lambda) g(y)
$$

for given functions $f, g: I \rightarrow \mathbb{R}(I \subset \mathbb{R}$ is an interval), for all $x, y \in I$, and for arbitrary $\lambda \in[0,1]$. In this sense the following statement is well-known.

Theorem 13. (Baron-Matkowski-Nikodem, [1994]). Real functions $f, g$ : $D \rightarrow \mathbb{R}(D$ is a convex subset in a linear space) satisfying the following 
inequality in the form as

$$
f\left(\sum_{j=1}^{n} \lambda_{j} x_{j}\right) \leq \sum_{j=1}^{n} \lambda_{j} g\left(x_{j}\right)
$$

for all $n \in \mathbb{N}$, for all $x_{1}, \ldots, x_{n} \in D$ and for reals $\lambda_{1}, \ldots, \lambda_{n} \in[0,1]$ with the property $\lambda_{1}+\cdots+\lambda_{n}=1$ if and only if there exists a convex function $h: D \rightarrow \mathbb{R}$ such that

$$
f(x) \leq h(x) \leq g(x) \quad \text { for every } \quad x \in D .
$$

This statement are proved B aron, Matkowski and Nikodem [1994]. A similar statement may be found in: Cholewa [1984]. Also, a result of M a t kow ski [1990] is connected with this.

Otherwise, an interesting illustration of Theorem 11 is well-known Hadamard's bilateral inequality. Namely, if $f: J \rightarrow \mathbb{R}(J \rightarrow \mathbb{R}$ is an open interval) is a convex function and if $a<b(a, b \in J)$, then holds the following bilateral inequality

$$
f\left(\frac{a+b}{2}\right) \leq \frac{1}{b-a} \int_{a}^{b} f(x) d x \leq \frac{f(a)+f(b)}{2} .
$$

This inequalities to demonstrate, adequate to Theorem 11, since in this case is form of the existing convex function $h: J \rightarrow \mathbb{R}$. This function, in this case for $f=g$ and when $f$ is convex by Jensen, has a concrete form. Theorem 11 gave only fact that this function existing.

For further facts on Hadamard's bilateral inequality of the form (Ha) see: T a s k ovi ć [2001]. For other further facts of this see: C h olew a [1984].

Extension of Mazur-Orlicz theorem. Let $X$ be a linear space over $\mathbb{K}(:=\mathbb{R}$ or $\mathbb{C})$. The set of all linear mappings $h: X \rightarrow \mathbb{K}$, in this section, denoted by $X^{*}$. The following extension of Hahn-Banach theorem is fundamental.

Theorem 14. (Mazur-Orlicz, [1953]). Let $X$ be a real linear space, $p: X \rightarrow$ $\mathbb{R}$ is a sublinear functional, $J$ is an arbitrary index set, $\left\{x_{j}: j \in J\right\} \subset X$, and let $\left\{c_{j}: j \in J\right\} \subset \mathbb{R}$. Then necessary and sufficient that there exists a linear functional $h \in X^{*}$ such that

$$
h \leq p, \quad c_{j} \leq h\left(x_{j}\right) \quad \text { for } \quad j \in J
$$

is the following condition: for every finite index set $\{j(1), \ldots, j(n)\} \subset J$ and for arbitrary non-negative numbers $\left\{\alpha_{1}, \ldots, \alpha_{n}\right\}$ the following inequality holds in the form as

$$
\sum_{k=1}^{n} \alpha_{k} c_{j(k)} \leq p\left(\sum_{k=1}^{n} \alpha_{k} x_{j(k)}\right) .
$$

We notice that there exist many applications of this statement. Otherwise, the inequality (MO) is well-known as Mazur-Orlicz condition, i.e., as a criterion for the consistency of the preceding system (HB). The following result is an extension of the preceding statement. 
Theorem 15. (Tasković, [2005]). Let $X$ be a real linear space and let $g$ : $X \rightarrow \mathbb{R}$ be a given general convex functional. If $J$ is an index set, $\left\{x_{j}: j \in\right.$ $J\} \subset X$, and if $\left\{c_{j}: j \in J\right\} \subset \mathbb{R}$, then the following system in the form as

$$
h \leq g, \quad c_{j} \leq h\left(x_{j}\right) \quad \text { for } \quad j \in J
$$

has at least one solution $h \in X^{*}$ if and only if for every finite index set $\{j(1), \ldots, j(n)\} \subset J$ and for arbitrary non-negative numbers $\left\{\alpha_{1}, \ldots, \alpha_{n}\right\}$ the following inequality holds in the form as

$$
\sum_{k=1}^{n} \alpha_{k} c_{j(k)} \leq g\left(\sum_{k=1}^{n} \alpha_{k} x_{j(k)}\right) .
$$

We notice that in the special case of this statement if $g: X \rightarrow \mathbb{R}$ is a sublinear functional (i.e., subadditive and homogeneous), then directly it follows the case of Mazur-Orlicz characterization of existing extensions of linear functionals.

Also, if $g: X \rightarrow \mathbb{R}$ is a convex and widdening a general convex function, then from Theorem 15 it follows directly two extensions of Hahn-Banach theorem by Kolmogorov (for convex functions) and by Tasković (for general convex functions).

In connection with the preceding we can say that the preceding sttement give necessary and sufficient conditions for solution an infinite system of inequalities (Sh) on a conjugate space of a given space.

\section{Transversal LOWER NORMED SPACES}

Let $X$ be a linear space over $\mathbb{K}$. The mapping $x \mapsto\|x\|: X \rightarrow[a, b]$ (or $x \mapsto\|x\|: X \rightarrow(a, b]$ ) for some $0 \leq a<b \leq+\infty$ is called a lower transversal seminorm (or lower seminorm) iff: $\|x\| \geq a$ for every $x \in X$, $\|\lambda x\|=|\lambda|\|x\|$ for all $\lambda \in \mathbb{K}$ and $x \in X$, and if there is a function $d$ : $[a, b]^{2} \rightarrow[a, b]$ such that

$$
\|x+y\| \geq \min \{\|x\|,\|y\|, d(\|x\|,\|y\|)\}
$$

for all $x, y \in X$.

Further, $x \mapsto\|x\|$ is called a lower transversal norm (or lower norm) iff in addition: $\|x\|=b$ if and only if $x=0$.

A lower transversal normed space $(X,\|\cdot\|)$ over $\mathbb{K}$ consists of a linear space $X$ over $\mathbb{K}$ together with a lower transversal norm $x \mapsto\|x\|$.

The function $d:[a, b]^{2} \rightarrow[a, b]$ in (Nd) is called lower bisection function. From $(\mathrm{Nd})$ it follows, by induction, that there is a function $d$ : $[a, b]^{n} \rightarrow[a, b]$ such that

$$
\begin{gathered}
\left\|x_{0}-x_{n}\right\| \geq \\
\geq \min \left\{\left\|x_{0}-x_{1}\right\|, \ldots,\left\|x_{n-1}-x_{n}\right\|, d\left(\left\|x_{0}-x_{1}\right\|, \ldots,\left\|x_{n-1}-x_{n}\right\|\right)\right\}
\end{gathered}
$$

for all $x_{0}, x_{1}, \ldots, x_{n} \in X$ and for any fixed integer $n \geq 1$. 
It is easy to verify that the lower transversal normed linear space $X$ is a transversal lower space (see: Tasković [2005]) with respect to the lower transverse $\rho: X \times X \rightarrow[a, b]$ defined by

$$
\rho[x, y]=\|x-y\| \quad \text { for all } \quad x, y \in X
$$

thus we obtain $\rho[x-z, y-z]=\rho[x, y]$ and $\rho[\lambda x, \lambda y]=|\lambda| \rho[x, y]$ for all $x, y, z \in X$ and for every scalar $\lambda \in \mathbb{K}$.

In this sense, the sequence $\left\{x_{n}\right\}_{n \in \mathbb{N}}$ in $(X,\|\cdot\|)$ converges (or lower converges) to $x \in X$ if the sequence $\left\{x_{n}\right\}_{n \in \mathbb{N}}$ converges (or lower converges) in $(X, \rho)$, i.e., if

$$
\rho\left[x_{n}, x\right]=\left\|x_{n}-x\right\| \rightarrow b \quad \text { as } \quad n \rightarrow \infty .
$$

In this sense, a lower transversal normed space $X$ is said to be lower complete (or complete) if it is lower complete as a transversal lower space.

We will, in further, denote by $\mathfrak{D}([a, b])$ the set of all lower bisection functions $d:[a, b]^{2} \rightarrow[a, b]$ which are increasing satisfying $d(t, t) \geq t$ for every $t \in[a, b]$.

We notice that lower transversal norm $x \mapsto\|x\|$ is a general concave function. The proof is simple.

Example 3 (Lower intervally normed space). Let $X$ be a linear space over $\mathbb{K}$ and let $\mathfrak{J}$ be the set of all nondecreasing, leftcontinuous functions $x \mapsto N_{x}: \mathbb{R} \rightarrow[a, b]$ for $a<b\left(a, b \in \mathbb{R}_{+}^{0}\right)$, where $\inf N_{x}=a$ and $\sup N_{x}=b$. Then $(X, \mathfrak{J})$ is a lower transversal normed space if: $N_{x}(0)=b$ for every $x \in X, N_{x}=a$ for $x \leq 0$ and $N_{x}=b$ for $x>0$,

$$
N_{\lambda x}(\sigma)=N_{x}\left(\frac{\sigma}{|\lambda|}\right) \quad \text { for every } \quad x \in X
$$

and for all $\sigma \in \mathbb{R}$ and $\lambda \neq 0$, and if there is a function $d:[a, b]^{2} \rightarrow[a, b]$ for $a, b \in \mathbb{R}_{+}^{0}(a<b)$ such that the following inequality holds in the forma as

$$
N_{x+y}(p+q) \geq \min \left\{N_{x}(p), N_{y}(q), d\left(N_{x}(p), N_{y}(q)\right)\right\}
$$

for all $x, y \in X$ and for all $p, q \geq 0$. For further facts and examples see: T a s k o v i ć[2005].

Example 4 (The space $L B(S)$ ). Let $S$ be a nonempty set and let $L B(S)$ be a set of all lower bounded functions $x: S \rightarrow \mathbb{R}$. Thus we have that $L B(S) \subset \mathbb{R}^{S}$ with the lower transversal norm defined by

$$
\|x\|=\inf \{|x(t)|: t \in S\} ;
$$

and we denote the resulting lower transversal normed space by $L B(S)$. Then $L B(S)$ is a lower complete lower transversal normed space from Proposition 33. (A brief poof of this fact may be found in: Tas k o vi ć[2005]).

If in the preceding definition of trasversal lower normed spaces the condition of homogenity of the form: $\|\lambda x\|=|\lambda|\|x\|$ for all $\lambda \in \mathbb{K}$ and $x \in X$ is to exchange with the condition of the form: $\|\lambda x\|=|f(\lambda)|\|x\|$ for all $\lambda \in \mathbb{K}, x \in X$, and a function $f: \mathbb{K} \rightarrow \mathbb{K}$, then we have a transversal 
lower global normed space $(X,\|\cdot\|)$, where $x \mapsto\|x\|$ is a transversal lower global norm.

Adequate we obtain a transversal lower global semi-normed space as and transversal lower global seminorm. On the other hand, in a lower transversal normed space $X$, with the lower bisection function $d \in \mathfrak{D}([a, b])$, the following inequality holds as

$$
\left\|\sum_{k=1}^{n} x_{k}\right\| \geq \min \left\{\left\|x_{1}\right\|, \ldots,\left\|x_{n}\right\|\right\}
$$

for all points $x_{1}, \ldots, x_{n} \in X$. (The proof of this inequality by induction may be found in: Taskovi ć[2005].)

Example 5. The linear space $\mathbb{R}^{k}$ of all $k$-tuples $x=\left(\xi_{1}, \xi_{2}, \ldots, \xi_{k}\right)$ of real numbers can be made into transversal lower normed linear space in an infinite variety of ways, as we shall see below. If the transversal lower norm on $\mathbb{R}^{k}$ is defined by

$$
\|x\|=\left\{\sum_{v=1}^{k}\left|\xi_{v}\right|^{2 / 3}\right\}^{3 / 2},
$$

then we get the $k$-dimensional Euclidean and transversal lower normed space familiar to us from our earlier work. Also, the inequality $(\mathrm{Nd})$ holds because for $y=\left(\eta_{1}, \eta_{2}, \ldots, \eta_{k}\right)$ the following inequality holds in the form as

$$
\|x+y\| \geq \min \left\{\|x\|,\|y\|,\left(\sum_{v=1}^{k}|| \xi_{v}|-| \eta_{v}||^{2 / 3}\right)^{3 / 2}\right\} .
$$

Example 6. Let $\mathbb{R}^{k}$ (or $\mathbb{C}^{k}$ ) be a set of all $k$-tuples $x=\left(\xi_{1}, \xi_{2}, \ldots, \xi_{k}\right)$ of real (complex) numbers can be made into transversal lower normed linear spaces if the transversal lower norm is defined by

$$
\|x\|=\left\{\sum_{v=1}^{k}\left|\xi_{v}\right|^{-2}\right\}^{-1 / 2},
$$

then we get the transversal lower normed spaces $\mathbb{R}^{k}$ (or $\mathbb{C}^{k}$ ). In this cases inequality (Nd) for $y=\left(\eta_{1}, \eta_{2}, \ldots, \eta_{k}\right)$ is satisfied from the following inequality in the form as

$$
\|x+y\| \geq \min \left\{\|x\|,\|y\|,\left(\sum_{v=1}^{k}|| \xi_{v}|-| \eta_{v}||^{-2}\right)^{-1 / 2}\right\} .
$$

Example 7 (Gauss-Petrović space). Let $a$ be a real number and let $0<\theta<$ $\pi / 2$. Let $\mathbb{R}_{\text {arg }}^{k}$ be set of all $k$-tuples $x=\left(\xi_{1}, \xi_{2}, \ldots, \xi_{k}\right)$ of complex numbers such that the following condition holds in the form as

$$
a-\theta \leq \arg \left(\xi_{v}\right) \leq a+\theta \quad(v=1, \ldots, k) ;
$$


then we get the transversal lower norm on $\mathbb{R}_{\text {arg }}^{k} \cup\{(0,0, \ldots, 0)\}$ in the following adequate form as

$$
\|x\|=\left\{\sum_{v=1}^{k}\left|\xi_{v}\right|^{p}\right\}^{1 / p} \quad \text { for } \quad-\infty<p<1 \quad(p \neq 0),
$$

i.e., the vector space $G P:=\left(\mathbb{R}_{\text {arg }}^{k}\right) \cup\{0\}$, where $0=(0,0, \ldots, 0)$ with the transversal lower norm of the form (27), is a transversal lower normed space.

Otherwise, the inequality $(\mathrm{Nd})$ holds in this case from Minkowski's inequality and from inequality known as Petrovićs complementary inequality triangle.

Annotations. In a matter honor of names $\mathrm{Carl} \mathrm{Fried} \mathrm{rich} \mathrm{G} \mathrm{a} \mathrm{s} \mathrm{s} \mathrm{and}$ Mihailo N. Petrović the preceding space GP has give name: Gauss-Petrović lower normed space. Indeed, de facto, GP-space there exists from the following inequality in 1917 of P etrović in the following variant: If (26) holds, then

$$
\left|\sum_{v=1}^{k} \xi_{v}\right| \geq(\cos \theta) \sum_{v=1}^{k}\left|\xi_{v}\right| \text {. }
$$

The Petrović's proof of this inequality is given by Gauss theorem of centroid. Inequality (28) is a complementary triangle inequality. It is difficult to say where it appeared for the first time in literature. We have found (see a historical review in M i t r i n o vi ć [1970]) that the special case $a=\theta=\pi / 4$ was proved by $\mathrm{P}$ e t r o vi ć [1917]. The general case of this inequality appears in a later paper of Petrović [1970]. He also applied (28) to derive some inequalities for integrals. Inequality (28) can be found also in: Karamata's book [1949, pp. 300-301]. This result is connected to Đoković's inequality by: M i t r i n o v i ć - P e č a r i ć - F i n k [1993].

The inequality (28) has been rediscovered by W i l f [1963], while the following variant of inequality (28) for $\theta=\pi / 2$ is given in $\mathrm{M}$ a r d e $\mathrm{n} \mathrm{[1949].} \mathrm{A} \mathrm{refiment}$ of inequality (3) is given by Vas ić-J a n i ć-K e č k i ć [1972] in the following form that if $a<\arg \left(\xi_{v}\right)<a+\theta$ for $v=1, \ldots, k$ then

$$
\left|\sum_{v=1}^{k} \xi_{v}\right| \geq \max \left(\frac{\sqrt{2}}{2}, \cos \theta\right) \sum_{v=1}^{k}\left|\xi_{v}\right| .
$$

Example 8 (The space $C[a, b]$ ). The points $x$ of the transversal lower normed space $C[a, b]$ are in closed segment $[a, b]$ for $b-a<+\infty$ continuous functions $x(t)$ with the transversal lower norm in the form as

$$
\|x\|=\min _{a \leq t \leq b}|x(t)| .
$$

Example 9 (The space $d l_{p}$ for $p \in \mathbb{R} \backslash\{0\}$ ). The points $x$ of transversal lower normed space $d l_{p}$ are infinite sequences of numbers $\left(\xi_{v}\right)$ such that the series $\sum_{v=1}^{\infty}\left|\xi_{v}\right|^{p}$ is divergent. In this space a transversal lower norm is defined by

$$
\|x\|=\left\{\sum_{v=1}^{n}\left|\xi_{v}\right|^{p}\right\}^{1 / p} \quad \text { for } \quad n \in \mathbb{N}
$$


where $x=\left(\xi_{1}, \xi_{2}, \ldots\right)$. It is easy to see that all facts hold for properties of the transversal lower norm, as and inequality $(\mathrm{Nd})$, for $y=\left(\eta_{1}, \eta_{2}, \ldots\right)$ in the form as

$$
\|x+y\| \geq \min \left\{\|x\|,\|y\|,\left(\sum_{v=1}^{n}|| \xi_{v}|-| \eta_{v}||^{p}\right)^{1 / p}\right\} .
$$

Example 10 (The space $h l_{p}(-\infty<p<1, p \neq 0)$ ). The points $x$ of transversal lower normed space $h l_{p}$ are infinite sequences of numbers $\left(\xi_{v}\right)$ such that $\sum_{v=1}^{\infty}\left|\xi_{v}\right|^{p} \geq$ $\mu$ for some $\mu>0$, and for a zero vector. In this space a transversal lower norm is defined by

$$
\|x\|=\inf _{n \in \mathbb{N}}\left\{\sum_{v=1}^{n}\left|\xi_{v}\right|^{p}\right\}^{1 / p},
$$

where $x=\left(\xi_{1}, \xi_{2}, \ldots\right)$. It is easy to see that all facts hold for properties of the transversal lower norm as and an inequality of the form $(\mathrm{Nd})$.

Example 11 (The space $L_{p, \infty}(a, b)$ for $\left.p \in \mathbb{R} \backslash\{0\}\right)$. Let $L_{p, \infty}(a, b)$ for $p \in \mathbb{R} \backslash\{0\}$ be a set of all measurable functions $x$ derined on $(a, b)$ such that $|x(t)|^{p}$ is an integrable function. In this vector space common with unit and zero-vector, $x+y$ and $\lambda x$ being respectively the mappings $(x+y)(t)=x(t)+y(t)$ and $(\lambda x)(t)=\lambda x(t)$, where a transversal lower norm defined by

$$
\|x\|=\left(\int_{a}^{b}|x(t)|^{p} d t\right)^{1 / p}
$$

for every $x \in L_{p, \infty}(a, b)$ and for $p \in \mathbb{R} \backslash\{0\}$. It is easy to see that all properties of transversal lower norm hold. The only axiom: $\|x\|=b \leq+\infty$ is equivalent to $x=0$ is not trivially verified. On the other hand, (Nd) holds in the form

$$
\|x+y\| \geq \min \left\{\|x\|,\|y\|,\left(\int_{a}^{b}|| x(t)|-| y(t)||^{p} d t\right)^{1 / p}\right\},
$$

for all $x, y \in L_{p, \infty}$ and for $p \in \mathbb{R} \backslash\{0\}$. Otherwise, as and in former cases, the inequality $(\mathrm{Nd})$ can be in further different forms.

Some more. (The space $L_{p, \infty}$ for $p \in \mathbb{R} \backslash\{0\}$ ). Let $\Omega$ be a nonempty set, $\Sigma$ is a $\sigma$-algebra of all subsets of $\Omega$, and let $\mu$ be a nonnegative nontrivial somplete and $\sigma$-finite measure on $\Omega$. An $L_{p, \infty}$ space essentially consists of all measurable functions $x$ such that

$$
\int_{\Omega}|x|^{p} d \mu=+\infty, \quad \text { for } \quad p \in \mathbb{R} \backslash\{0\},
$$

with the transversal lower norm defined corresponding as in (29). Also, in this case hold all conditions for lower norm.

Further facts. From the preceding properties for the space $L_{p, \infty}$ in the special case, if $p<1(p \neq 0)$, then from the following inequality in the form as

$$
\left(\int_{a}^{b}(|x|+|y|)^{p} d t\right)^{1 / p} \geq\left(\int_{a}^{b}|x|^{p} d t\right)^{1 / p}+\left(\int_{a}^{b}|y|^{p} d t\right)^{1 / p}
$$


it follows directly the following fact in the form that if $x, y \in L_{p, \infty}(a, b)$, then $|x|+$ $|y| \in L_{p, \infty}(a, b)$. Otherwise, from the classical metric inequality $|x \pm y| \geq|| x|-| y||$ for arbitrary $p \in \mathbb{R} \backslash\{0\}$ it follows that $x \pm y \in L_{p, \infty}(a, b)$ if $|x|-|y| \in L_{p, \infty}(a, b)$.

On the other hand, from Hölder's inequality (problem 4.2) for $p<1(p \neq 0)$ in the case for two measurable functions in the following form as

$$
\int_{\Omega}|f g| d \mu \geq\|f\|_{p}\|g\|_{q} \text { for } \quad \frac{1}{p}+\frac{1}{q}=1
$$

it follows directly that $f g \in L_{1, \infty}$, in the case if $f \in L_{p, \infty}$ and $g \in L_{q, \infty}$, or in the case if $f \in L_{p, \infty}$ and $g \in L_{q}$, or in the case if $f \in L_{p}$ and $g \in L_{q, \infty}$.

In connection with a result of W. O r l i c z in 1932 (: if $\sum_{n=1}^{\infty} d_{n}^{2}=+\infty$, then there exists a continuous function such that $\sum_{n=1}^{\infty}\left|a_{n}\right|\left|d_{n}\right|=+\infty$ via a condition in the form that the orthonormal system of function $\left(\varphi_{n}\right)$ on $(a, b)$ is infinite and contained of uniformly bounded functions), from inequality (30) we obtain the following result:

If $p<1(p \neq 0)$ and if $f \in L_{p, \infty}$ then there exists a function $g \in L_{q, \infty}$ (or $\left.g \in L_{q}\right)$ for $1 / p+1 / q=1$ such that $f g \in L_{1, \infty}$.

An annotation. We notice that from the preceding Orlicz's result in 1932 directly it follows that there exists a continuous function for which the following series in the form as

$$
\sum_{n=1}^{\infty}\left|a_{n}\right|^{2-\varepsilon}
$$

is divergent for every $\varepsilon>0$. This redult is an analogy with singularity in the case of trigonometrical systems which first time considered $\mathrm{C}$ a r l e m a n. Also, this result is connected directly with the object of parameters convergence different systems introduced by: Tasković in 1971. In connection with this see: A d a m ović [1968].

In further we notice that every lower intervally normed space is a transversal lower intervally space if a transverse $\rho[x, y]=N_{x-y}$. Let $\tau(D)$ be a topology for $b<+\infty$ in the following sense that

$$
D_{x}(\varepsilon)=\left\{y \in X: N_{x-y}(\varepsilon)>b-\varepsilon\right\}
$$

for every $x \in X$ and for every $\varepsilon>0$. In the next set $B(x)=a$ for $x \leq 0$ and $B(x)=b$ for $x>0$. Now we can formulate the following result.

Proposition 21. Let $X$ be a vector space over $\mathbb{K}(:=\mathbb{R}$ or $\mathbb{C}), N_{x}(0)=b$ for every $x \in X, N_{x}=B$ if and only if $x=0, N_{\alpha x}(\varepsilon) \geq N_{x}(\varepsilon)$ for $0<|\alpha|<1$, $N_{\alpha x}(\varepsilon) \rightarrow b$ as $\alpha \rightarrow 0$, and

$N_{x-y}(\varepsilon)>b-\varepsilon \quad$ and $\quad N_{y-z}(\delta)>b-\delta \quad$ implies $\quad N_{x-z}(\varepsilon+\delta)>b-(\varepsilon+\delta)$

for all $\varepsilon, \delta>0$, then $(X, \tau(D))$ is a transversal topological vector space, where the lower transverse $\rho$ has the following form as

$$
\rho[x, y]= \begin{cases}0 \text { if } y \in D_{x}(\varepsilon) & \text { for every } \varepsilon>0 \\ \sup \left\{\varepsilon: y \notin D_{x}(\varepsilon)\right. & \text { for every } 0<\varepsilon<1,\end{cases}
$$


in agreement with translation and invariance of the lower transverse in the form: $\rho[0, \lambda x] \leq \rho[0, x]$ for $|\lambda|<1$. Also, $\rho[x, y]<\tau$ if and only if $N_{x-y}(\tau)>$ $b-\tau$ for every $\tau>0$.

Proof. Set $\mathfrak{G}=\left\{D_{x, y}=N_{x-y}: x, y \in X\right\}$, then directly, from the preceding facts we obtain the following fact that

$D_{x, y}(\varepsilon)>b-\varepsilon \quad$ and $\quad D_{y, z}(\delta)>b-\delta$ implies $D_{x, z}(\varepsilon+\delta)>b-(\varepsilon+\delta)$ for all $\varepsilon, \delta>0$. On the other hand, the condition (32) implies the following condition in the form as: for every $\varepsilon>0$ there exists $0<\delta \leq \varepsilon$ such that

$$
D_{x, y}(\delta)>b-\delta \quad \text { and } \quad D_{y, z}(\delta)>b-\delta \text { implies } D_{x, z}(\varepsilon)>b-\varepsilon,
$$

where the condition (33) is to equivalent with the fact that $(X, \tau(D))$ is a topological vector space, i.e., the first part of statement holds. also, from the fact

$$
\rho[0, x-z]=\sup \left\{\varepsilon: N_{0-(x-z)}(\varepsilon)=N_{x-z}(\varepsilon) \leq b-\varepsilon\right\}=\rho[x, z]
$$

it follows that $\rho[x+y, y+z]=\rho[0, x+y-(y+z)]=\rho[0, x-z]=\rho[x, z]$. In this context, for $|\lambda| \leq 1$ the following fact holds as

$$
\rho[0, \lambda x]=\sup \left\{\varepsilon: N_{\lambda x}(\varepsilon) \leq b-\varepsilon\right\} \leq \sup \left\{\varepsilon: N_{x}(\varepsilon) \leq b-\varepsilon\right\}=\rho[0, x]
$$

i.e., from these facts we obtain that the lower transverse is invariance and translation. Also holds and secont part of statement because $\tau(D)=\tau(\mathfrak{G})$. The proof is complete.

We notice that if $(X, \mathfrak{R})$ is a lower transversal intervally normed space with a lower bisection function $d \in \mathfrak{D}([a, b])$, then (31) holds, because for $N_{x-y}(\varepsilon)>b-\varepsilon$ and $N_{y-z}(\delta)>b-\delta$ we obtain that

$$
\begin{gathered}
N_{x-z}(\varepsilon+\delta)=N_{x-y+y-z}(\varepsilon+\delta) \geq \\
\geq \min \left\{N_{x-y}(\varepsilon), N_{y-z}(\delta), d\left(N_{x-y}(\varepsilon), N_{y-z}(\delta)\right)\right\} \geq \\
\geq \min \left\{N_{x-y}(\varepsilon), N_{y-z}(\delta), d\left(\min \left\{N_{x-y}(\varepsilon), N_{y-z}(\delta)\right\}, \min \left\{N_{x-y}(\varepsilon), N_{y-z}(\delta)\right\}\right)\right\} \geq \\
\geq \min \left\{N_{x-y}(\varepsilon), N_{y-z}(\delta), \min \left\{N_{x-y}(\varepsilon), N_{y-z}(\delta)\right\}\right\}> \\
>\min \{b-\varepsilon, b-\delta, \min \{b-\varepsilon, b-\delta\}\}>b-(\varepsilon+\delta) .
\end{gathered}
$$

Proposition 22. Let $S$ be an arbitrary set and let $L B(S)$ be a lower transversal linear normed space of all lower bounded real functions $x: S \rightarrow \mathbb{R}$ with the lower norm $\|x\|=\inf \{|x(t)|: t \in S\}$. Then $L B(S)$ is a lower complete space. 
Proof. The proof that $L B(S)$ is a transversal lower normed space is standard. We shall proved that $L B(S)$ is lower complete. In this sense let $\left(x_{n}\right)$ be a fundamental sequence in $L B(X)$. Then for every $\varepsilon>0$ there exists $n_{0} \in \mathbb{N}$ such that $\left\|x_{n+k}-x_{n}\right\|>\varepsilon$ for all $n, k \geq n_{0}$. On the other hand, for every $\varepsilon>0$ for every $t \in S$ and for all $n, k \geq n_{0}$ we obtain that

$$
\begin{gathered}
\left|x_{n+k}(t)-x_{n}(t)\right|=\left|\left(x_{n+k}-x_{n}\right)(t)\right| \geq \\
\geq \inf \left\{\left|\left(x_{n+k}-x_{n}\right)(t)\right|: t \in S\right\}=\left\|x_{n+k}-x_{n}\right\|>\varepsilon .
\end{gathered}
$$

hence for every $t \in S$ the sequence of real numbers $\left(x_{n}(t)\right)$ is a fundamental sequence in the space $\mathbb{R} \subset \overline{\mathbb{R}}$. Since $\overline{\mathbb{R}}$ is a lower complete space, it follows that for every $t \in S$ the sequence $\left(x_{n}(t)\right)$ is lower converges to $x_{0}(t) \in \overline{\mathbb{R}}$, i.e., for the function $x_{0}: S \rightarrow \mathbb{R}$ we have $x_{0}(t)=\lim _{n \rightarrow \infty} x_{n}(t)$ for every $t \in S$. For $k \rightarrow \infty$ from (34) we obtain

$$
\left|x_{0}(t)-x_{n}(t)\right| \geq \varepsilon \text { for every } t \in S
$$

and thus $\inf \left\{\left|x_{0}(t)-x_{n}(t)\right|: t \in S\right\} \geq \varepsilon$ for $n \geq n_{0}$, i.e., this means that $x_{0}-x_{n}$ is a lower bounded function for $n \geq n_{0}$, i.e., $x_{0}-x_{n} \in L B(S)$ and $\left\|x_{0}-x_{n}\right\| \geq \varepsilon$.

Since $x_{0}=\left(x_{0}-x_{n}\right)+x_{n}$ and $x_{0}-x_{n}, x_{n} \in L B(S)$, and since $L B(S)$ is a linear space, it follows that $x_{0} \in L B(S)$ and $\lim _{n \rightarrow \infty} x_{n}=x_{0}$ in the space $L B(S)$. The proof is complete.

Proposition 23. Let $X$ be a linear space and let $(X, \rho)$ be a lower transversal space, then lower transverse $\rho$ can be defined with lower norm if and only if the following facts hold:

$$
\begin{gathered}
\rho[x-a, y-a]=\rho[x, y] \quad \text { for all } \quad x, y, a \in X, \\
\rho[\lambda x, \lambda y]=|\lambda| \rho[x, y] \quad \text { for all } \quad x, y \in X \text { and } \lambda \in \mathbb{K} .
\end{gathered}
$$

Proof. Let $f: X \rightarrow \mathbb{R}$ be a defined by $f(x)=\rho[0, x]$ for $x \in X$, then $f$ is a lower transversal norm on $X$. Indeed, let $\lambda \in \mathbb{K}$, then from (36) it follows that

$$
f(\lambda x)=\rho[0, \lambda x]=\rho[\lambda 0, \lambda x]=|\lambda| \rho[0, x]=|\lambda| f(x),
$$

i.e., $f$ is an absolute homogeneous function. On the other hand, from (35) and given conditions, it follows that for all $x, y \in X$ we have the following inequality in the form

$$
\begin{gathered}
f(x+y)=\rho[0, x+y]=\rho[-x, y] \leq \\
\leq \max \{\rho[-x, 0], \rho[0, y], d(\rho[-x, 0], \rho[0, y])\}=\max \{f(x), f(y), g(f(x), f(y))\},
\end{gathered}
$$

which means that the function $f$ satisfy the corespondent inequality for lower transvrse, i.e., $f$ is a lower (transversal) norm on $X$. Otherwise,

$$
\rho[x, y]=\rho[0, y-x]=f(y-x) \text { for all } x, y \in X,
$$


and thus, from the fact (36), it follows that $\rho[x, y]=f(x-y)$, i.e., the lower transverse $\rho$ is defined with the lower norm $f$. The proof is complete.

Annotation. Every two open (closed) balls, as and every two spheres, in a transversal lower normed space $X$ are homeomorphic. Unhurt space, also, is homeomorphic to an arbitrary proper open ball. Thus in $X$ are essential the balls and spheres with center 0 and radius 1 , i.e., $d(K(0,1)), d(K[0,1])$, and $d(S(0,1))$.

If the lower bisection function $d \in \mathfrak{D}([a, b])$, then every ball is a convex set; also, for different of lower transversal spaces, we have that $\mathrm{Cl}(d(K(a, r)))=$ $d(K[a, r])$ and $\operatorname{Int}(d(K[a, r]))=d(K(a, r))$ for $a \in X$ and $r>0$.

If $x \mapsto\|x\|$ is a lower norm on the vector space $E$, then $\rho[x, y]=\|x-y\|$ is a transverse on $E$ such that $\rho[x+z, y+z]=\rho[x, y]$ and $\rho[\lambda x, \lambda y]=|\lambda| \rho[x, y]$ for any scalar $\lambda \in \mathbb{K}$.

Theorem 16. Let $F$ be a closed linear subspace of a lower transversal normed space $E$ with the bisection function $d \in \mathfrak{D}([a, b])$ and let

$$
\|x+F\|=\sup \{\|x+y\|: y \in F\}
$$

for all $x \in F$. The mapping $(x+F) \mapsto\|x+F\|$ is a lower transversal norm on $E / F$, and further, if $E$ is lower complete, so is $E / F$.

The proof of this statement is founded on the facts of problems 1 and 2 . A proof for this statement may be found in: Ta s k o v i ć[2005].

The lower transversal normed spaces $\left(X,\|\cdot\|_{X}\right)$ and $\left(Y,\|\cdot\|_{Y}\right)$ with the same fiels $\mathbb{K}$ are isometric isomorphism (or isomorphism) if there exists a one-to-one linear transformation (operator) $A: X \rightarrow Y$ such that

$$
\|A x\|_{Y}=\|x\|_{X} \quad \text { for every } \quad x \in X
$$

and $X$ is said to be isometrically isomorphic to $Y$ if there exists an isometric isomorphism of $X$ onto $Y$.

This terminology enables us to give precise meaning to the statement that one lower transversal normed linear space is essentially the same as another.

In this sense, if the lower transversal normed spaces $X$ and $Y$ are isometrically isomorphic and if one of him complete (lower complete), then other of him also complete space.

Linear lower bounded operators. Let $X$ and $Y$ be lower transversal normed spaces over $\mathbb{K}$ and let $L(X, Y)$ be a set of all linear operators of $X$ into $Y$. An opertor $A \in L(X, Y)$ is bounded (or lower bounded) if there exists a real number $\mu>0$ with the property that

$$
\|A x\| \geq \mu\|x\| \text { for every } \quad x \in X ;
$$

in this case, then $\mu$ is called a bound (or lower bound) for $A$, and such an $A$ is often referred to as a bounded linear operator, where a lower norm 
of operator $A$, in notation $\|A\|$, defined by

$$
\|A\|:=\inf _{x \in X \backslash\{0\}} \frac{\|A x\|}{\|x\|}
$$

or on a second manner, as supremum of the numbers $\mu>0$ for which holds (37). Otherwise, the set $S \subset X$ is bounded (or lower bounded) if there exists $m>0$ such that $\|x\| \geq m$ for every $x \in S$.

In this sense from (38) we can obtain and other formulas for $\|A\|$. Specially, for some $\mu \in \mathbb{R}_{+}$holds $\|A\| \geq \mu$ if and only if $\|A x\| \geq \mu\|x\|$ for every $x \in X$. Set $\mu=\|A\|$ then it follows that $\|A x\| \geq\|A\|\|x\|$ for every $x \in X$.

In further, the set of all linear lower bounded operators of $X$ into $Y$ denoted by $L B(X, Y)$; also, the space $L B(X, \mathbb{K})$ is denoted by $X^{*}$ and is called the conjugate or more briefly, functionals.

On the other hand, from (37) is $\inf _{x \in X \backslash\{0\}}\left(\|A x\|\|x\|^{-1}\right) \geq \mu$, and thus the following inequality holds in the form as

$$
\|A x\| \geq\left(\inf _{x \in X \backslash\{0\}} \frac{\|A x\|}{\|x\|}\right)\|x\| \quad \text { for every } \quad x \in X,
$$

which means that, from all given facts in this chapter of the book, the following facts hold, if $A: X \rightarrow Y$ is a homogeneous operator, in this case the following equalities in the form as

$$
\begin{gathered}
\|A\|=\inf _{\|x\| \geq 1}\|A x\|=\inf _{\|x\|=1}\|A x\|=\inf _{x \in X \backslash\{0\}} \frac{\|A x\|}{\|x\|}= \\
=\max \left\{\mu \in \mathbb{R}_{+}:\|A x\| \geq \mu\|x\| \quad \text { for every } \quad x \in X\right\},
\end{gathered}
$$

where $\max \varnothing=0$; and hence, directly, we obtain the following fact: the operator $A: X \rightarrow Y$ is bounded (or lower bounded) if and only if the following inequality holds in the form as

$$
\inf _{x \in X \backslash\{0\}} \frac{\|A x\|}{\|x\|}>0 .
$$

Illustrations. The identity mapping of $X$ into itself $\operatorname{id}_{X}: X \rightarrow X$ defined by $\operatorname{id}_{X}(x)=x$ for $x \in X$ is linear. Since $\left\|\operatorname{id}_{X}(x)\right\|=\|x\|$ for every $x \in X$, it follows $\left\|\operatorname{id}_{X}\right\|=1$.

Also, if $X$ is a subspace of the space $Y$, then the mapping $A_{X}: X \rightarrow Y$ defined by $A_{X}(x)=x$ for $x \in X$ is linear and $\left\|A_{X}\right\|=1$ (for $X \neq\{0\}$ ).

Among the rest, if the operator $A: X \rightarrow Y$ is a homogeneous operator, then for it lower norm the following facts hold in the next as

$$
\|A\|=\inf _{x \in X \backslash\{0\}} \frac{\|A x\|}{\|x\|}=\inf _{x \in X \backslash\{0\}}\left\|\frac{1}{\|x\|} A x\right\|=\inf _{x \in X \backslash\{0\}}\left\|A\left(\frac{x}{\|x\|}\right)\right\|,
$$

hence, because of the fact that for every $x \in X \backslash\{0\}$ the lower norm or the vector $x /\|x\|$ is equaly 1 , it follows fact that $\|A\|=\inf _{\|x\|=1}\|A x\|$, which is 
one of the equalities in (39). Since

$$
\inf _{\|x\|=1}\|A x\|=\|A\| \leq \inf _{\|x\| \geq 1}\|A x\| \leq\|A\|,
$$

we obtain that the first equality in (39) holds. This with (40) give a good reason for the preceding equalities in (39).

Proposition 24. Let $X$ and $Y$ be lower transversal normed spaces and $A$ : $X \rightarrow Y$ a linear operator. Then the following conditions are all equivalent to one another: 1) $A$ is lower continuous, 2) $A$ is lower continuous at the origin (in the sense that $x_{n} \rightarrow 0$ implies that $A\left(x_{n}\right) \rightarrow 0$ ), 3) $A$ is lower bounded, and 4) if $d(S(0,1)):=\{x:\|x\| \geq 1\}$ is closed unit sphere in $X$, then its image $A(d(S(0,1)))$ is a lower bounded set in $Y$.

Remarks. We notice that if an additive operator is lower continuous in a point of space, then it is lower continuous on the entire space. For an additive operator $A: X \rightarrow Y$ the following two facts hold: $A(0)=0$ and $A(-x)=-A(x)$ for every $x \in X$.

Proof of Proposition 24 First, the condition of lower uniformly continuous implies lower continuous of the mapping $A: X \rightarrow Y$ in the point $0 \in X$. On the other hand, if $A \in L B(X, Y)$, then for $\delta=\varepsilon / \mu$ and $\mu, \varepsilon>0$ from the inequality $\|x-y\|>\delta$ it follows that

$$
\|A x-A y\| \geq \mu\|x-y\|>\varepsilon,
$$

i.e., this means that $A$ is lower uniformly continuous on the space $X$. Reverse, if $A$ is lower continuous in $0 \in X$, then $A \in L B(X, Y)$.

Indeed, if $A$ is lower continuous in $0 \in X$, then for $\varepsilon=1$ there exists $\delta>0$ such that $\|A x\|>1$ whenever $\|x\|>\delta$. Hence, for $x \in X(x \neq 0)$ and $0<\delta<\alpha$ it follows that $\|\alpha x /\| x\|\|>\delta$ and that

$$
\|A x\|=\left\|\frac{\|x\|}{\alpha} A\left(\frac{\alpha x}{\|x\|}\right)\right\| \geq \frac{1}{\alpha}\|x\| ;
$$

and thus, since this inequality holds and for $x=0$, it follows that $A \in$ $L B(X, Y)$. This means from all the preceding facts that 2 ) is equivalent to 3).

On the other hand, since a nonempty subset of a lower normed linear space is lower bounded iff it is contained in a closed sphere centered on the origin, it is evident that 3) implies 4); for if $\|x\| \geq 1$, then $\|A x\| \geq \mu$. to show that 4$)$ implies 3$)$, we assume that $A(d(S(0,1)))$ is contained in a closed sphere of radius $\mu$ centered on the origin. If $x=0$, then $A(x)=0$, and clearly $\|A x\| \geq \mu\|x\|$; and if $x \neq 0$, then $x /\|x\|$ in $d(S(0,1))$, and therefore $\|A(x /\|x\|)\| \geq \mu$, so again we have $\|A x\| \geq \mu\|x\|$. The proof is complete.

Proposition 25. Let $X$ and $Y$ be transversal lower normed spaces and let $A: X \rightarrow Y$ be a linear operator. Then $A$ is a lower bounded operator if and only if every lower bounded set in $X$ maps in some lower bounded set in $Y$. 
Proof. Necessity. Let the operator $A: X \rightarrow Y$ be a lower bounded and let $S$ be a lower bounded set, i.e., $\|A\|>0$ and $\|x\| \geq \mu>0$ for every $x \in S$, then it follows that

$$
\|A x\| \geq\|A\|\|x\| \geq \mu\|A\| \quad \text { for every } \quad x \in S .
$$

Sufficiently. If $d(K)$ is closed unit sphere in $X$, then its image $A(d(K))$ is a lower bounded set in $Y$, i.e., there exists a number $\mu>0$ such that $\|A x\| \geq \mu$ for every $x \in d(K)$. Let $x \in X \backslash\{0\}$ be an arbitrary point, then $x /\|x\| \in d(K)$ such that

$$
\left\|A \frac{x}{\|x\|}\right\| \geq \mu, \quad \text { i.e., } \quad\|A x\| \geq \mu\|x\|,
$$

but, since this inequality holds and for $x=0$, from this fact it follows that $A$ is a lower bounded operator. The proof is complete.

Example 12. Let $x=\left(\xi_{v}\right) \in l_{p}$ for $-\infty<p<1(p \neq 0)$ and let $\left[\alpha_{v \mu}\right]$ be an infinite matrix of numbers such that the following inequality holds in the form as

$$
\sum_{\mu=1}^{\infty} \sum_{v=1}^{\infty}\left|\alpha_{v \mu}\right|^{q}<+\infty\left(\frac{1}{p}+\frac{1}{q}=1\right)
$$

then with the following corresponding equalities in the form as in the next adequate forms with the equality that is

$$
\eta_{\mu}=\sum_{v=1}^{\infty}\left|\alpha_{v \mu} \xi_{v}\right| \quad(\mu=1,2, \ldots)
$$

is defined a lower bounded operator $y=A x$ for $x=\left(\xi_{v}\right)$ and $y=\left(\eta_{\mu}\right)$ which maps the space $l_{p}$ in the space $l_{q}$ with $-\infty<p<1(p \neq 0)$ and $1 / p+1 / q=1$. Indeed, since from Hölder's inequality the following inequality holds in the form as

$$
\left|\eta_{\mu}\right|=\sum_{v=1}^{\infty}\left|\alpha_{v \mu} \xi_{v}\right| \geq\left\{\sum_{v=1}^{\infty}\left|\alpha_{v \mu}\right|^{q}\right\}^{1 / q}\left\{\sum_{v=1}^{\infty}\left|\xi_{v}\right|^{p}\right\}^{1 / p}
$$

for $\mu \in \mathbb{N}$, hence to put power $q$ for this inequality and to bring together left and right side of this inequality for $\mu=1,2, \ldots$ we obtain the following inequality in the form as

$$
\|y\|_{l_{q}} \geq\left\{\sum_{\mu=1}^{\infty} \sum_{v=1}^{\infty}\left|\alpha_{v \mu}\right|^{q}\right\}^{1 / q}\|x\|_{l_{p}},
$$

hence it follows that the following fact holds that is $y \in L_{q}$, i.e., the operator $A$ maps the space $l_{p}$ in the space $l_{q}$ and the following inequality holds in the form as

$$
\|A\| \geq\left\{\sum_{\mu=1}^{\infty} \sum_{v=1}^{\infty}\left|\alpha_{v \mu}\right|^{q}\right\}^{1 / q},
$$

i.e., the oprator $A$ is a lower bounded operator. If $x=\left(\xi_{v}\right)$ is a sequence of nonnegative numbers, then $A$ is a linear operator. The proof for this is elementary. 
Example 13. Let $K(s, t)$ be a measure function which is defined on the unit square $Q=\{(s, t): 0 \leq s, t \leq 1\}$ and let the following inequality holds in the form as

$$
\int_{0}^{1}|K(s, t)| d t \geq D \quad \text { for every } \quad s \in[0,1],
$$

then with the following equality in the form as in the next that is $y(s)$ is an integral in the following sense that is with

$$
y(s)=\int_{0}^{1}|K(s, t) x(t)| d t
$$

is defined a lower bounded operator $y=A x$ for $x=x(t)$ and $y=y(s)$ which maps the space of the essential bounded functions $M(0,1)$ into itself. Indeed, from the following inequalities in the form as

$$
|y(s)| \geq(\operatorname{infess} 0 \leq t \leq 1|x(t)|) \int_{0}^{1}|K(s, t)| d t \geq D\|x\|_{M},
$$

it follows that

$$
\text { infess } 0 \leq s \leq 1|y(s)| \geq D\|x\|_{M},
$$

i.e., $\|A x\|_{M} \geq D\|x\|_{M}$. Hence we obtain $A x \in M(0,1), A$ is a lower bounded operator and $\|A\| \geq D$. The linearity for $A$ is not satisfied. But, if $x=x(t)$ is a nonnegative function, then $A$ is a linear operator. The proof for this is elementary.

Lower locally compactness. A topological space $X$ is said to be locally compact if for every point $x \in X$ there exists a compact neighbourhood of $x$ in $X$. Any discrete space is locally compact, but not compact. The real line $\mathbb{R}$ is locally compact but not compact.

A lower transversal normed space $X$ is said to be lower locally compact if for every point $x \in X$ there exists a lower compact neighbourhood of $x$ in $X$.

Proposition 26. The transversal lower normed space $X$ is lower locally compact if and only if the closed unit ball of the form as

$$
d(K[0,1]):=\{x \in X:\|x\| \geq 1\}
$$

is a lower compact set. (The proof of this statement is a totally analogy with the proof of Proposition 10).

Proof. Let $d(K[0,1])$ is a lower compact set. Since for every point $x_{0} \in X$ the mapping $x \mapsto x+x_{0}$ is homeomorphism of $X$ into $X$, the sets $x_{0}+d(K[0,1])$ and $d(K[0,1])$ are homeomorphic sets, and thus $x_{0}+d(K[0,1])$ is a lower compact neighbourhood of the point $x_{0}$.

Reverse, let $X$ be a lower locally compact space, thus the point $0 \in X$ has a lower compact neighbourhood $V$ which is a closed set and contained a ball $V(0, r)$ for $r>0$.

The set $d(K[0,1])=C l(V(0, r)) \subset \mathrm{Cl}(V)=V$ is lower compact (as a closed subset of lower compact set $V)$. Hence, the closed unit ball $d(K[0,1])$ is an image of the set $d(K[0, r])$ under the continuous mapping $x \mapsto(1 / r) x$ 
such that thus also $d(K[0,1])$ is a lower compact set. The proof is complete.

We notice, from the preceding facts, that a nonempty bounded closed subset of a finite dimensional lower transversal normed linear space is lower compact. There is a converse of this fact which is true and which provides an important characterization of finite dimensional lower transversal normed linear spaces. We shall need an essential statement.

Proposition 27. (Geometrical lemma). Let $X$ be a lower transversal normed space and let $Y$ be a linear subspace of $X$ that is a proper closed subset of $X$. Then for every $\varepsilon>1$ there exists a point $x_{\varepsilon} \in X$ such that

$$
\left\|x_{\varepsilon}\right\|=1 \quad \text { and } \operatorname{hdiam}\left(x_{\varepsilon}, Y\right):=\sup _{y \in Y}\left\|x_{\varepsilon}-y\right\|<\varepsilon .
$$

Proof. From the former facts and from given conditions of statement directly it follows that there exists a point $x_{0} \in X \backslash Y$ such that

$$
\operatorname{hdiam}\left(x_{\varepsilon}, Y\right)=\sup _{y \in Y}\left\|x_{0}-y\right\|:=d>0 .
$$

Since $d<d \varepsilon$ (for every $\varepsilon>1$ ), hence there exists a point $z \in Y$ such that $d / \varepsilon<\left\|x_{0}-z\right\| \leq d$. If we choice that $x_{\varepsilon}=\left(x_{0}-z\right) /\left\|x_{0}-z\right\|$, then $\left\|x_{\varepsilon}\right\|=1$. Also, in this case, for $y \in Y$ we obtain

$$
\left\|x_{\varepsilon}-y\right\|=\frac{\left\|x_{0}-\left(z+y\left\|x_{0}-z\right\|\right)\right\|}{\left\|x_{0}-z\right\|} \leq \frac{d}{\left\|x_{0}-z\right\|}<\varepsilon,
$$

which means, common with all preceding facts, that statement holds. The proof is complete.

Further application of Proposition 46 we have that the lower compactness of closed unit ball is sufficiently for finite dimensional of a transversal lower normed space.

Theorem 17. Let $X$ be a lower transversal normed space and let $d(K[0,1])=$ $\{x \in X:\|x\| \geq 1\}$ be a closed lower compact ball in $X$, then the lower transversal normed space $X$ is finite dimensional.

Proof. (Application of Geometrical lemma). Let $d(K[0,1])$ be a lower compact set in $X$ and let $X$ be an infinite dimensional space. Suppose that $x_{1} \in X,\left\|x_{1}\right\|=1$ and $X_{1} \equiv \operatorname{Lin}\left(\left\{x_{1}\right\}\right)$; then $X_{1}$ is a proper closed subspace in $X$. Also, there exists $x_{2} \in X$ such that $\left\|x_{2}\right\|=1$ and $\operatorname{hdiam}\left(x_{2}, X_{1}\right)<2$ (from Proposition 46), which means that is $\left\|x_{2}-x_{1}\right\|<2$.

Let $X_{2} \equiv \operatorname{Lin}\left(\left\{x_{1}, x_{2}\right\}\right)$, then $X_{2}$ is a proper closed subspace in $X$. Again, from Proposition 46, there exists $x_{3} \in X$ such that $\left\|x_{3}\right\|=1$ and $\operatorname{hdiam}\left(x_{3}, X_{2}\right)<2$, i.e., this means that $\left\|x_{3}-x_{2}\right\|<2$, as and $\left\|x_{3}-x_{1}\right\|<2$.

Applying Proposition 46, via method of mathematical induction, it follows that there exist a sequence of finite dimensional subspaces $\left\{X_{n}\right\}_{n \in \mathbb{N}}$ in $X$ 
and a sequence $\left\{x_{n}\right\}_{n \in \mathbb{N}}$ in $X$ such that

$$
X_{1} \subsetneq X_{2} \subsetneq \cdots, x_{n} \in X,\left\|x_{n}\right\|=1 \text { and } \operatorname{hdiam}\left(x_{n+1}, X_{n}\right)<2
$$

for every $n \in \mathbb{N}$. Thus ist follows that $\left\|x_{n}-x_{m}\right\|<2$ for $n \neq m$, which means that the sequence $\left\{x_{n}\right\}_{n \in \mathbb{N}}$ has not the lower convergent subsequences. This is a contradiction with the supposition that $d(K[0,1])$ is a lower compact subset in $X$. The proof is complete.

From the proof of the preceding statement as and from Proposition 46 we have an immediately consequence in the following form as.

Corollary 2. Let $X$ be a lower transversal normed space and let the sphere $d(S(0,1))=\{x \in X:\|x\|=1\}$ be a lower compact subset in $X$, then the lower transversal normed space $X$ is finite dimensional.

Otherwise, on the lower transversal normed space also holds a spring form of Riesz's lemma in the following from as.

Proposition 28. (Form of Riesz lemma). Let $X$ be a lower transversal normed space and let $Y$ be a linear subspace of $X$ that is proper closed subset of $X$. Then for every $\varepsilon \in(0,1)$ there exists a point $x_{\varepsilon} \in X$ such that

$$
\left\|x_{\varepsilon}\right\|=1 \quad \text { and } \quad \rho\left[x_{\varepsilon}, Y\right]:=\inf _{y \in Y}\left\|x_{\varepsilon}-y\right\|>\varepsilon .
$$

The proof of this statement is adequate to the proof which is has had Riesz [1918] for the case of classical normed space.

An annotation. We notice that Geometrical lemma and Form of Riesz lemma de facto hold on upper and lower transversal normed spaces. But, for geometry of lower normed spaces is essential Geometrical lemma, till for geometry of upper normed spaces is essential Form of Riesz lemma.

We turn now to the question of the existence of nonzero lower bounded linear functionals on an arbitrary nonzero lower transversal normed space. Such functionals obviously exist when the space is one-dimensional and every nonzero linear space has one-dimensional linear subspaces. In this section we derive a general extension statement.

Theorem 18. Let $X$ be a real linear space, let $D \subset X$ be a subspace, and let $x \mapsto\|x\|: X \rightarrow \mathbb{R}$ be a lower transversal norm such that

$$
h(x) \geq\|x\| \text { for every } x \in D,
$$

where $h: D \rightarrow \mathbb{R}$ is a linear functional. Then there exist at least countable or finite linear functionals $H_{k}: X \rightarrow \mathbb{R}$ such that

$$
H_{k}(x) \geq\|x\| \text { for every } x \in X
$$

and

$$
H_{k}(z)=h(z) \quad \text { for every } \quad z \in D \text {. }
$$


Lemma 3. Let $X$ be a linear space, let $D \subset X$ be a set $Q$-convex and $Q$ radial at a point $x_{0} \in D$, and let $L \subset X$ be a linear space (over $\mathbb{Q}$ ) such that $x_{0} \in L$. Let $f: D \rightarrow \mathbb{R}$ be a function fulfilling the inequality

$$
f(\lambda x+(1-\lambda) y) \geq \min \{f(x), f(y), d(f(x), f(y))\}^{5}
$$

for a function $d: f(D)^{2} \rightarrow \mathbb{R}$, for all $x, y \in D$ and for every $\lambda \in \mathbb{Q} \cap[0,1]$. If $z \notin L$, if $Z=\operatorname{Lin}(L \cup\{z\})$ and if $h: L \rightarrow \mathbb{R}$ is a linear functional such that

$$
h(x) \geq f(x) \quad \text { for every } \quad x \in D \cap L,
$$

then there exists a linear functional $H: Z \rightarrow \mathbb{R}$ such that the following inequality holds in the form as

$$
H(x) \geq f(x) \quad \text { for every } \quad x \in D \cap Z
$$

J and $H \mid L=h$, i.e., there is an extension of the linear functional $h$ on $Z$.

Proof. For all $x, y \in L$ and for all $\lambda, \mu \in \mathbb{Q} \cap(0, \infty)$ such that $x+\mu z \in D$ and $y-\lambda z \in D$ we obtain from (M) and (41) the following inequalities in the form as

$$
\begin{gathered}
\frac{\lambda}{\lambda+\mu} h(x)+\frac{\mu}{\lambda+\mu} h(y)=h\left(\frac{\lambda}{\lambda+\mu} x+\frac{\mu}{\lambda+\mu} y\right) \geq \\
\geq f\left(\frac{\lambda}{\lambda+\mu} x+\frac{\mu}{\lambda+\mu} y\right)=f\left(\frac{\lambda}{\lambda+\mu}(x+\mu z)+\frac{\mu}{\lambda+\mu}(y-\lambda z)\right) \geq \\
\geq \min \{f(x+\mu z), f(y-\lambda z), d(f(x+\mu z), f(y-\lambda z))\} ;
\end{gathered}
$$

and thus, over directly calculation, we obtain the following correspondent inequality of the form as

$$
\begin{gathered}
\frac{h(x)-f(x+\mu z)}{\mu} \geq \frac{1}{\lambda}\left[\frac{\lambda+\mu}{\mu} \min \{f(x+\mu z), f(y-\lambda z),\right. \\
\left.d(f(x+\mu z), f(y-\lambda z))\}-h(y)-\frac{\lambda}{\mu} f(x+\mu z)\right]:=R ;
\end{gathered}
$$

and defined $U=\{(x, \mu) \in L \times \mathbb{Q}: \mu>0, x+\mu z \in D\}$ and $V=\{(y, \lambda) \in$ $L \times \mathbb{Q}: \lambda>0, y-\lambda z \in D\}$ from the preceding inequality we obtain the

${ }^{5}$ General concave functions. In an earlier paper (Task ović: Math. Japonica, 37 (1992), 367-372), introduced the notion of general concave functions. A function $f: D \rightarrow \mathbb{R}$, where $\mathbb{R}$ denotes the real line and $D$ is a convex subset of $\mathbb{R}^{n}$, is said to be general concave iff there is a function $\psi:(f(D))^{2} \rightarrow \mathbb{R}$ such that

$$
f(\lambda x+(1-\lambda) y) \geq \min \{f(x), f(y), \psi(f(x), f(y))\}
$$

for all $x, y \in D$ and for arbitrary $\lambda \in[0,1]$. We notice that the set of all concave and quasi concave functions can be a proper subset of the set of all general concave functions. We notice that the lower transversal norm $x \mapsto\|x\|$ is a general concave function. The proof is simple. 
following inequality in the form as in the next

$$
N:=\frac{h(x)-f(x+\mu z)}{\mu} \geq \sup _{V} R,
$$

which means that $\beta:=\sup _{U} N \geq \sup _{U} \sup _{V} R:=\alpha$.

Since $D$ is $Q$-radial at $x_{0}$, we have $\left(x_{0}, \mu\right) \in U$ for $\mu \in \mathbb{Q} \cap(0, \varepsilon(z))$ and $\left(x_{0}, \lambda\right) \in V$ for $\lambda \in \mathbb{Q} \cap(0, \varepsilon(-z))$. Consequently $U \neq \varnothing$ and $V \neq \varnothing$. Observe also that $\alpha \leq \beta$ implies that $-\infty<\alpha \leq \beta<+\infty$. In particular $[\alpha, \beta] \neq \varnothing$.

Choose any $c \in[\alpha, \beta]$. Every $t \in Z$ may be uniquely written as $t=x+\lambda z$, where $x \in L$ and $\lambda \in \mathbb{Q}$. For such a $t \in Z$ define $H(t)=h(x)-c \lambda$. It is easily seen that $H: Z \rightarrow \mathbb{R}$ is a linear functional and that $H \mid L=h$. Now take an arbitrary $t \in D \cap Z$ which may be represented in form $t=x+\lambda z$. Consider three cases:

1) If $\lambda=0$, then $t=x$; and by inequality (41) we obtain $H(t)=h(x) \geq$ $f(x)=f(t)$. Consequently this fact we have that inequality $(\mathrm{H})$ holds.

2) If $\lambda>0$, then since $t \in D$ we have $(x, \lambda) \in U$. Since $c<\beta$, this inequality implies $h(x)-f(x+\lambda z) \geq c \lambda$, i.e., the following want fact $(\mathrm{H})$ holds in the form as

$$
H(t)=h(x)-c \lambda \geq f(x+\lambda z)=f(t) .
$$

3) If $\lambda<0$, then since $t \in D$ we have $(x,-\lambda) \in V$. Since $\alpha<c$, this inequality implies that the following inequality holds in the form as

$$
\begin{gathered}
-c \lambda \geq \frac{-\lambda+\mu}{\mu} \min \{f(x+\mu z), f(x+\lambda z), d(f(x+\mu z), f(x+\lambda z))\}- \\
-h(x)+\frac{\lambda}{\mu} f(x+\mu z),
\end{gathered}
$$

and thus, in the context of fact since is defined functional $H(t)$, directly for $\mu=\lambda$ we obtain the following want fact $(\mathrm{H})$ in the form as

$$
H(t)=h(x)-c \lambda \geq f(x+\lambda z)=f(t),
$$

i.e., the inequality $(\mathrm{H})$ holds in this case, too. Now, together with all the preceding facts, the proof is complete.

Hence we derive a totally new form of the rational version of the well known Hahn-Banach theorem.

Theorem 19. Let $X$ be a real linear space, let $D \subset X$ be a set $Q$-convex and $Q$-radial at a point $x_{0} \in D$, and let $L \subset X$ be a linear space (over $\mathbb{Q}$ ) such that $x_{0} \in L$. Let $f: D \rightarrow \mathbb{R}$ be a function fulfilling $(M)$ for all $x, y \in D$ and for every $\lambda \in \mathbb{Q} \cap[0,1]$. If $h: L \rightarrow \mathbb{R}$ is a linear functional with property (41), then there exist at least countable or finite linear functionals $H_{k}: X \rightarrow \mathbb{R}$ such that

$$
H_{k}(x) \geq f(x) \quad \text { for every } \quad x \in D
$$


and $H_{k} \mid L=h$, i.e., there exist at least countable or finite extensions of the linear functional $h$ on $X$ of $L$.

In this section arguing as in the proof of Theorem 33 we can get however the following result.

Theorem 20. (Form of Hahn-Banach theorem). Let $X$ be a real linear space, let $D \subset X$ be a subspace and let $f: X \rightarrow \mathbb{R}$ be a general concave function such that

$$
h(x) \geq f(x) \quad \text { for every } \quad x \in D,
$$

where $h: D \rightarrow \mathbb{R}$ is a linear functional. Then there exist at least countable or finite linear functionals $H_{k}: X \rightarrow \mathbb{R}$ such that

$$
H_{k}(x) \geq f(x) \quad \text { for every } \quad x \in X
$$

and $H_{k} \mid D=h$, i.e., there exist at least countable or finite linear extensions of the linear functional $h$ on the space $X$.

A brief proof of Theorem 20 (as and Lemma 3 and Theorem 19) may be found in Tasković [2005].

Proof of Theorem 18. Applying Theorem 20 for the case when $f(x)=$ $\|x\|: X \rightarrow \mathbb{R}$, where $x \mapsto\|x\|$ is a lower transversal norm, directly we obtain this statement.

We notice that Theorem 20 guarantees that a lower transversal normed space is richly supplied with continuous linear functionals, and makes possible an adequate theory of conjugate spaces.

Proof of Theorem 20. Let $\mathfrak{R}$ be the family of all couples $(Y, A)$, where $Y$ is a linear space (over $\mathbb{Q}$ ), $L \subset Y \subset X$, and $A: Y \rightarrow \mathbb{R}$ is a linear functional such that $A \mid L=h$ and

$$
A(x) \geq f(x) \text { for all } x \in D \cap Y,
$$

where $(L, h) \in \mathfrak{R}$, so $\mathfrak{R}$ is a nonempty family. We introduce the order in $\mathfrak{R}$ in the usual manner: $\left(Y_{1}, A_{1}\right),\left(Y_{2}, A_{2}\right) \in \mathfrak{R}$ we agree that $\left(Y_{1}, A_{1}\right) \preccurlyeq\left(Y_{2}, A_{2}\right)$ if $Y_{1} \subset Y_{2}$ and $A_{2} \mid Y_{1}=A_{1}$. If $Z \subset \Re$ is any chain, then put

$$
G=\bigcup_{(Y, A) \in Z} Y,
$$

and define $B: G \rightarrow \mathbb{R}$ putting $B(y)=A(y)$ if $y \in Y$ and $(Y, A) \in Z$. The couple $(G, B)$ is an upper bound of $Z \in \mathfrak{R}$. In fact, if $x, y \in G$ and $\alpha \in \mathbb{Q}$, then there exists an $(Y, A) \in Z$ such that $x, y \in Y$. Then also $x+y \in Y \subset G$ and $\alpha x \in Y \subset G$, which shows that $G$ is a linear space (over $\mathbb{Q}$ ). Since $L \subset Y \subset X$ for all $Y$ such that $(Y, A) \in Z$, also $L \subset G=\bigcup_{(Y, A) \in Z} Y \subset X$. Similarly it is shown that $B$ is a linear functional fufilling (43) and such that $B \mid L=h$.

By Lemma of Infinite Maximality in $\mathfrak{R}$ there exist at least countable or finite maximal elements $\left(E_{k}, H_{k}\right)$. The only thing we need to show is that 
$E_{k}=X$. Supposing the contrary, let $z \in X \backslash E_{k}$. By Lemma 3 there exists a linear functional $H_{k}^{*}: \operatorname{Lin}\left(E_{k} \cup\{z\}\right) \rightarrow \mathbb{R}$ such that $H_{k}^{*} \mid E_{k}=H_{k}$, whence $H_{k}^{*}\left|L=H_{k}\right| L=h$, and

$$
H_{k}^{*}(x) \geq f(x) \text { for every } \quad x \in D \cap \operatorname{Lin}\left(E_{k} \cup\{z\}\right),
$$

and consequently this $\left(\operatorname{Lin}\left(E_{k} \cup\{z\}\right), H_{k}^{*}\right) \in \mathfrak{R}$, and clearly $\left(E_{k}, H_{k}\right) \prec$ $\left(\operatorname{Lin}\left(E_{k} \cup\{z\}\right), H_{k}^{*}\right)$, which contradicts maximality of $\left(E_{k}, H_{k}\right)$. Consequently we must have $E_{k}=X$. The proof is complete.

The following form of open mapping theorem enables us to give a satisfactory description of the projections on a lower transversal normed space, an has the important closed graph statement as one of its consequences.

Theorem 21. (Form of the open mapping theorem). Let $X$ and $Y$ be lower complete lower transversal normed spaces with continuous lower norms and with a bisection function $d \in \mathfrak{D}\left(\mathbb{R}_{+}^{0}\right)$. If $A$ is a lower bounded linear operator of $X$ onto $Y$, then $A$ is an open mapping, i.e., $A(G)$ is an open subset of $Y$ whenever $G$ is an open subset of $X$.

As an immediately consequence of the Form of the open mapping theorem it follows the following statement on bounded inverse operator in the following form as.

Theorem 22. (Statement of inverse bounded). Let $X$ and $Y$ be two lower complete transversal lower normed spaces with continuous lower norms and bisection function $d \in \mathfrak{D}([a, b])$. If $A \in B(X, Y)$ and if $A$ is bijective, then there exists an inverse operator $A^{-1}$ such that $A^{-1} \in B(X, Y)$.

Proof. Let $A$ be a one-to-one continuous linear operator of $X$ onto $Y$. Then, by the Form of the open mapping theorem, $A(G)$ is an open subset of $Y$ whenever $G$ is an open subset of $X$. Since $A$ is one-to-one we have

$$
\left(A^{-1}\right)^{-1}(G)=A(G)
$$

for every set $G$ in $X$, and consequently it follows from Theorem 21 that $A^{-1}$ is a continuous operator. The proof is complete.

Proposition 29. Let $X$ and $Y$ be lower complete trnasversal lower normed spaces with continuous lower norms and the bisection function $d \in \mathfrak{D}([a, b])$. If $A \in K(X, Y)$, then image operator not contained infinite dimensional closed subspace in $Y$.

Theorem 23. (Form of Banach - Steinhaus theorem). Let $J$ be an index set, $X$ and $Y$ be lower transversal normed spaces with coninuous lower norms and with a bisection function $d \in \mathfrak{D}\left(\mathbb{R}_{+}^{0}\right), X$ be a lower complete space and $A_{j} \in B(X, Y)$ for every $j \in J$. Then $\inf _{j \in J}\left\|A_{j} x\right\|>0$ for every $x \in X$ if and only if $\inf _{j \in J}\left\|A_{j}\right\|>0$.

Theorem 24. (Form of convergence principle). Let $X$ and $Y$ be lower transversal normed spaces with continuous lower norm and with a bisection 
function $d \in \mathfrak{D}\left(\mathbb{R}_{+}^{0}\right)$, $Y$ is a lower complete space and $\left\{A_{n}\right\}_{n \in \mathbb{N}}$ the sequence of lower bounded linear operators of $X$ into $Y$ such that:

1) $\left\|A_{n}\right\| \geq \mu$ for every $n \in \mathbb{N}$;

2) There exists $\lim _{n \rightarrow \infty} A_{n} x$ for all points $x \in D$ in a dense subset $D$ of a boll $K:=d\left(K\left(x_{0}, r\right)\right)$ in $X$.

Then, there exists $\lim _{n \rightarrow \infty} A_{n} x=A x$ for every $x \in X$, and $A$ is a lower bounded linear operator with the property $\|A\| \geq \mu$.

Proposition 30. (Form of principle of uniform boundedness). Let $X$ be a lower complete transversal lower normed space and $Y$ be a lower transversal normed space over the same field with bisection function $d \in \mathfrak{D}([a, b])$. If $\mathfrak{G}$ is a subset of $L(X, Y)$ with the property that $\{A x: A \in \mathfrak{E}\}$ is a lower bounded subset of $Y$ for each $x \in X$, then $\mathfrak{G}$ is a lower bounded subset of $L(X, Y)$.

The proof of this statement is a totally analogy with the proof of Theorem 4. A part of this proof is including in the proof of Banach-Steinhaus theorem. There is a special case of Proposition 30 which is sufficiently important to merit a separate statement.

Lower total continuous operators. Let $X$ and $Y$ be two lower transversal normed spaces. The linear operator $A: X \rightarrow Y$ is called lower total continuous iff every lower bounded set in $X$ maps in a relatively compact set in $Y$.

In this sense, every lower total continuous operator is a lower continuous operator. Namely, since every relatively compact set is a lower bounded set, hence lower total continuous operator maps lower bounded sets in lower bounded sets, which means that it lower continuous (from Proposition 25).

We notice that in an infinite dimensional lower transversal normed space the lower bounded operator not obliged will be lower total continuous.

An example, if $X$ is an infinite dimensional space, then the identical operator id: $X \rightarrow X$ (which is lower continuous) is not lower total continuous.

Indeed, by supposition in $X$ there exists infinite a great linear independence vectors $x_{1}, x_{2}, \ldots$; and let $X_{n}$ (for $n \in \mathbb{N}$ ) be a subspace which determined vectors $x_{1}, x_{2}, \ldots, x_{n}$. Then $X_{n}$ is a proper subset of $X_{n+1}$ and $X_{n}$ is closed (as a finite dimensional subspace). From Proposition 27 (Geometrical lemma) there exists a sequence of vectors $\left\{y_{n}\right\}_{n \in \mathbb{N}}$ such that

$$
\left\|y_{n}\right\|=1, \quad y_{n} \in X_{n} \quad \text { and } \operatorname{hdiam}\left(y_{n}, X_{n-1}\right)<2 \text {; }
$$

where the set $\left\{y_{n}\right\}_{n \in \mathbb{N}}$ is lower bounded in $X$. If an identical operator id is lower total continuous, it will be the set $\left\{y_{n}\right\}_{n \in \mathbb{N}}$ maps in a relatively compact set; but, $\operatorname{id}\left(\left\{y_{n}\right\}_{n \in \mathbb{N}}\right)=\left\{y_{n}\right\}_{n \in \mathbb{N}}$ which means that in this case of the sequence $\left\{y_{n}\right\}_{n \in \mathbb{N}}$ we can not to set spart a convergent subsequence because then $\left\|y_{m}-y_{n}\right\|<2$ for all $m, n \in \mathbb{N}(m \neq n)$. 
Proposition 31. Let $X$ be a lower transversal normed space. If $A: X \rightarrow X$ is a lower total continuous operator and $B: X \rightarrow X$ is a lower bounded operator, then $A B$ and $B A$ are lower total continuous operators.

Proof. First, if $M$ is a lower bounded set, then $B(M)$ is a lower bounded set, and thus $A(B M)$ is a relatively compact set (because lower total continuous of the operator $A$ ). This means that $A(B)$ is a lower total continuous operator.

Second, if $M$ is a lower bounded set, then $A(M)$ will be relatively compact (as and $B(A M)$ ), because $B$ is a lower continuous operator. Accordingly, $B(A)$ is a lower total continuous operator. The proof is complete.

Annotation. From Proposition 31 it follows that the lower total continuous operator $A: X \rightarrow X$ can not have lower bounded inverse operator! Indeed, if there exists a lower bounded inverse operator $A^{-1}$, then we obtain $A A^{-1}=$ id; and hence applying Proposition 31 it follows that id is a lower total continuous operator, which is not precisely.

Proposition 32. Let $X$ and $Y$ be complete lower transversal normed spaces with the bisection function $d \in \mathfrak{D}([a, b])$ and let $A_{n}: X \rightarrow Y(n=1,2, \ldots)$ be a sequence of the lower total continuous operators. If for the liner operator $A: X \rightarrow Y$ holds that

$$
\left\|A_{n}-A\right\| \rightarrow b \leq+\infty \quad(\text { as } n \rightarrow \infty),
$$

i.e., if the sequence of operators $\left\{A_{n}\right\}_{n \in \mathbb{N}}$ lower uniformly converge to the operator $A$, then $A$ is a lower total continuous operator.

An alternative for equations. We notice that is well-known the role of Riesz's lemma (Proposition 16) for a formulation of a form of famous Fredholm alternative in 1903 on integral equations.

Applying Geometrical lemma (Proposition 27) we obtain a result in the form an alternative (of lower total continuous linear operators) for operators equations.

Proposition 33. Let $X$ be a lower complete lower transversal normed space and let $A: X \rightarrow X$ be a lower total continuous linear operator. If the equation

$$
x-A x=y
$$

has a solution for every $y \in X$, then the following equation in a corresponding form as

$$
x-A x=0
$$

has a unique trivial solution. (An analogous statement of this also holds for the conjugate operators equations). 
We notice that if equation (46) has a solution for every $y \in X$, then this solution is unique. Indeed, if for some $y \in X$ there exist two different solutions $x_{1}$ and $x_{2}$, then from the equalities in the form as

$$
x_{1}-A x_{1}=y \quad \text { and } \quad x_{2}-A x_{2}=y
$$

it follows the equality $\left(x_{1}-x_{2}\right)-A\left(x_{1}-x_{2}\right)=0$ which is in contrariety with the preceding statement, because $x_{1}-x_{2} \neq 0$.

Proposition 34. Let $X$ be a lower complete lower transversal normed space and let $A: X \rightarrow X$ be a lower total continuous linear operator. If the equation

$$
x-A x=0
$$

has a trivial solution, then the following equation in a corresponding form as

$$
x-A x=y
$$

has a solution for every $y \in X$. (This statement is in some sense vice versa of the preceding statement.)

Further, a combination of the preceding two statements we obtain the following result as an alternative for operators equations.

Theorem 25. (Lower Fredholm alternative). Let $X$ be a lower complete lower transversal normed space and let $A: X \rightarrow Y$ be a lower total continuous linear operator. Then, or the equation

$$
x-A x=y
$$

has a unique solution for every $y \in X$, or the equation of the form $x-A x=0$ has a nontrivial solution.

Remark. A matter honor I have such that the preceding statement denoted to name by Ivar Fred holm (1866-1927) ${ }^{6}$ as lower Fredholm alternative. The results of his doctoral thesis in 1900 they have been in daybreak of Hilbert spaces.

\footnotetext{
${ }^{6}$ History of Hilbert spaces. One of the most active proponents of Fredholm's new theory on integral equations of 1900 was $\mathrm{D}$ a vid $\mathrm{Hil}$ b e r t. As soon as he heard of Fredholm's result, he started doing research himself on these questions, made them one of the main subject discussed in his Seminar in Göttingen, and supervised many dissertations on the various aspects of the theory. Between 1904 and 1906 he published six papers on integral equations in the "Göttinger Nachrichten" later brought together in a single volume entitled "Grundzüge einer allgemeinen Theorie der Integralgleichungen" (Teubner, Leipzig 1912). The results of Hilbert are but one step removed from what we now call the theory of Hilbert space; but the birth of this theory almost immediately followed the publication of Hilbert's papers (by René Maurice Fréchet and Erhard Schmidt in 1908). It seems to me that it is due to the fact that this publication precisely occurred during the emergence of a new concept in mathematics, the concept of structure. J o h n vo n N e u m a n n was the first to conceive of an "abstract" Hilbert space in 1929 (in connection with his spectral theory for unbounded hermitian operators and his investigations on the mathematical foundations of quantum mechanics.
} 
In further we notice that lower Fredholm alternative also holds and for the equations $\lambda x-A x=y$ and $\lambda x-A x=0$, where $\lambda \neq 0$ is an arbitrary number. Otherwise, Fredholm's first formulation of alternative it has been for the integral equations in the form

$$
\begin{gathered}
x(s)-\lambda \int_{0}^{1} K(s, t) x(t) d t=0, \\
x(s)-\lambda \int_{0}^{1} K(s, t) x(t) d t=y(s),
\end{gathered}
$$

and in his honor this equations to carry his name. Some later is formulated an alternative for equations via total continuous linear operators.

For a proof of Proposition 34 we have the following two half statements which are foundation on extension Hahn-Banach theorem (Theorem 20) in the following sense.

Proposition 35. Let $X$ be a lower complete lower transversal normed space and let $A: X \rightarrow X$ be a lower total continuous linear operator. If the functional $y^{*} \in X^{*}$ is to annul in every point $x \in X$ with the equation $x-A x=0$, then the equation

$$
x^{*}-A^{*} x^{*}=y^{*}
$$

has a solution. Specially, if the equation $x-A x=0$ has a unique trivial solution, then the equation (50) has a solution for every $y^{*} \in X^{*}$.

Proof. Let on the vector subspace $L$ which is composition of all vectors of the form $z=x-A x$ the functional $x^{*}$ defined by

$$
x^{*}(x-A x)=y^{*}(x) ;
$$

then, from Extension Hahn-Banach theorem (Theorem 20), we can $x^{*}$ (broaden on whole space $X$. If $T=$ id $-A$, then

$$
x^{*}(x-A x)=x^{*}((\mathrm{id}-A) x)=x^{*}(T x)=\left(T^{*} x^{*}\right) x=\left(\mathrm{id}^{*} x^{*}-A^{*} x^{*}\right) x,
$$

and thus, profitable (51), it follows that $x^{*}$ satisfying the equation (50). The proof is complete.

Proposition 36. Let $X$ be a lower complete lower transversal normed space and let $A: X \rightarrow X$ be a lower total continuous linear operator. If in the point $y \in X$ every the functional $x^{*} \in X^{*}$ is to annul with the property $x^{*}-A^{*} x^{*}=0$, then the following equation in the form as

$$
x-A x=y
$$

has a solution. Specially, if the equation $x^{*}-A^{*} x^{*}=0$ has a trivial solution, then the equation (52) has a solution for every $y \in X$.

Proof. Let $M^{*}$ be a set of all functionals $x^{*}$ on $X$ which satisfying the equation $x^{*}-A^{*} x^{*}=0$. Every $x^{*} \in M^{*}$ is corresponding the set $L_{x^{*}}$ of all points $x \in X$ with the property $x^{*}(x)=0$. If the vector $y \in X$ satisfying

$$
x^{*}(y)=0 \text { for every } x^{*} \in M^{*},
$$


then $y \in \bigcap_{x^{*} \in M^{*}} L_{x^{*}}$, which means that every vector $y$ can be written in the form $x-A x$. In this sense, let $x_{1}^{*}$ be a functional such that

$$
x_{1}^{*}(y) \neq 0 \quad \text { and } \quad x_{1}^{*}(x-A x)=0 \quad \text { for every } \quad x \in X,
$$

where $y_{1} \notin(\mathrm{id}-A) X$. In further, we first prove a construction of a lower bounded linear functional $x_{1}^{*}$ on $X$ such that (53) holds.

For the proof of this fact, let $L_{0}$ be a set of all vectors of the form $x-A x$. Let $\left\{L_{0}, y_{1}\right\}$ be a vector subspace of all vectors of the form $z+\alpha y_{1}$, where $z \in L_{0}$. Defined a lower bounded linear functional $x_{1}^{*}$ on $\left\{L_{0}, y_{1}\right\}$ such that

$$
x_{1}^{*}\left(z+\alpha y_{1}\right)=\alpha,
$$

and hence, from Extension Hahn-Banach theorem (Theorem 20), we can be broaden on whole space $X$. Since $x_{1}^{*}\left(y_{1}\right)=x_{1}^{*}\left(0+1 \cdot y_{1}\right)=1$ and since

$$
x_{1}^{*}(x-A x)=x_{1}^{*}\left((x-A x)+0 \cdot y_{1}\right)=0,
$$

hence it follows that the functional $x_{1}^{*}$ satisfying all facts of (53). The proof is complete.

Proof of Proposition 34. Immediately to combine of Propositions 35 and 36 we obtain that all facts hold in Proposition 34.

The proof of this statement is totally analogous with the proof of Theorem 25 based on Lemma of Infinite Maximality and the former characterization of reflexivity on lower transversal normed spaces. Thus we omit the proof.

Middle transversal normed spaces. In the preceding two part of this paper I have had two spaces (or two sides of a space): upper transversal normed space and lower transversal normed space. As a new space (or as third side of a given space) is a middle transversal normed space by Task ović[2005]. In this sense, a middle transversal normed space is an upper transversal normed space and a lower transversal normed space simultaneous.

\section{Miscellaneous ReSults, problems AND APPlicAtions}

3.1. Upper and Lower Compact Operators, T a s k o v i ć[2005]. The new facts on lower compact operators can play a central role in nonlinear functional analysis. Their importance stems from the fact that many results on lower continuous operators on $\mathbb{R}^{n}$ carry over to transversal lower normed spaces when "lower continuous" is replaced by "lower compact".

In connection with this, as it is well-known, compact and completely continuous operators occur in many problems of classical analysis. In the nonlinear case, the first comprehensive research on compact operators with numerous applications to partial differential equations (both linear and nonlinear) was due to J. Schauder: Zur Theorie stetiger Abbildungen in Funktionalräumen, Math. Zeit., 26 (1927), 47-65.

Literature on applications of the Schauder theorem to nonlinear problems is extensive. The first topological proof of the Peano theorem is due to G. D. Birkhoff and O. D. Kellogg: Invariant points in function space, Trans. AMS, 23 (1922), 95115. 
Juliusz Schauder in 1930 briefed: "The problem which this work brings to a certain conclusion was first investigated by Messrs. Birkhoff and Kellogg in 1922. These authors already recognized the correctness of the fixed point theorem in the simplest function spaces, but for each individual function spaces, the proof had to be carried anew from the beginning".

We notice that the fundamental idea of applying fixed point results to produce theorems in analysis is due to H. Poincaré: Sur certaines solutions particulières du problème des trois corps, Bull. Astronom., 1 (1884), 65-74.

The possibility of defining such notions as a limit and a continuity in an arbitrary set is an idea which undoubtedly was put forward for the first time by M. Fréchet in 1904, and developed by him in his famous thesis in 1906.

The simplest and most fruitful method which be proposed for such definitions was the introduction of the notion of distance.

But the greatest merit of Fréchet lies in the emphasis he put on three notions which were to play a fundamental part in all later developments of Functional Analysis: compactness, completeness, and separability.

In this sense, the notion of order, and the notion of new completeness, have each led to a fixed point statement. We now obtain geometric results of fixed points based on an interplay of these two notions as new notations in transversal upper and lower spaces.

In connection with this, first, in Tasković [1998] we introduced the concept of transversal (upper and lower) spaces as a natural extension of Fréchet's, Kurepa's, and Menger's spaces.

Further, let $X$ and $Y$ be Banach spaces and $T: D(T) \subset X \rightarrow Y$ an operator, which is called compact (or upper compact) iff: $T$ is continuous and $T$ maps bounded sets into relatively compact sets.

Compact operators play a central role in a nonlinear functional analysis. Their importance stems from the fact that many facts on continuous operators on $\mathbb{R}^{n}$ carry over to Banach spaces when continuous is replaced by compact.

For finite-dimensional Banach spaces, continuous and compact operators are the same whenever the domain $D(T)$ is closed. For if $S$ is bounded, then $\bar{S}$ is compact, since $\operatorname{dim}(X)<\infty$. Then $f(\bar{S})$ is compact, and hence $f(S)$ is relatively compact. Typical examples of compact operators on infinite dimensional Banach spaces are integral operators with sufficiently regular integrands. We have the following characterization of compact operators by S c h a u d e r [1934].

Proposition 37. (Approximation for compact operators). Let $X$ and $Y$ be Banach spaces and $M$ be a nonempty bounded subset of $X$. Then $T: M \rightarrow Y$ is a compact operator if and only if for every $n \in \mathbb{N}$ there exists a compact operator $P_{n}: M \rightarrow Y$ such that $\operatorname{dim}\left(\operatorname{Span} P_{n}(M)\right)<\infty$ and

$$
\sup _{x \in M}\left\|T(x)-P_{n}(x)\right\| \leq 1 / n .
$$

We notice that in the proof of this statement we essentially use the characterization of relatively compact sets in Banach spaces by finite $\varepsilon$-nets. In this sense, if $T$ is a compact operator, then $T(M)$ is relatively compact so that so-called Schauder operator defined by

$$
P_{n}(x)=\frac{\sum_{i=1}^{n} a_{i}(x) y_{i}}{\sum_{i=1}^{n} a_{i}(x)}
$$


where $a_{i}(x)=\max \left\{n^{-1}-\left\|T x-y_{i}\right\|, 0\right\}$, has all the requisite properties for the continuous $a_{i}(x)$ do not all vanish simultaneously for $x \in M$.

In the preceding chapter, we have the basic fixed-point theorems for compact operators in normed linear spaces. The approach is based on a general techniques as: The approximation of compact maps by finite-dimensional ones, which leads to the analogs of the Brouwer and Borsuk theorems for compact maps. Further, for immediate applications of Brouwer and Schauder fixed point theorems to nonlinear systems of equations, to system of inequalities, to nonlinear integral equations, and to ordinary and semilinear partial differential equations see: Z e i d l e r [1986], D u g undji-Granas [1982], and Tasković [1993].

Peano's Theorem. (Initial value problem). Further we give an application of General Schauder fixed point theorem to differential equations. As a parallel and contrast to the Picard-Lindelöf theorem we consider the initial value problem of the form as

$$
x^{\prime}(t)=f(t, x(t)), \quad x\left(t_{0}\right)=y_{0},
$$

on $\left[t_{0}-c, t_{0}+c\right]$. Geometrically, (54) means that we are looking for a curve which satisfies the differential equation and passes through $\left(t_{0}, y_{0}\right)$ as in Figure 6 with $y_{0}=p_{0}$. At the end points $t=t_{0} \pm c$, where $x^{\prime}(t)$ is to be interpreted as the appropriate one-sided derivative.

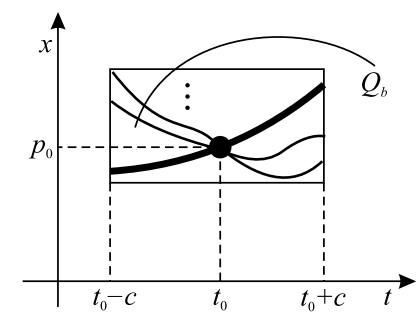

Figure 6

Proposition 38. (P e a n o [1890], Taskovi ć [2012]). Let there be given real numbers $t_{0}$ and $y_{0}$, and the rectangle of the form as

$$
Q_{b}:=\left\{(t, x) \in \mathbb{R}^{2}:\left|t-t_{0}\right| \leq a,\left|x-y_{0}\right| \leq b\right\},
$$

where $a$ and $b$ are fixed positive numbers. Suppose that $f: Q_{b} \rightarrow \mathbb{R}$ is continuous and bounded with the following condition of the form as

$$
|f(t, x)| \leq K \quad \text { for all } \quad(t, x) \in Q_{b},
$$

and fixed $K>0$. Set $c:=\min \{a, K / b\}$. Then the initial value problem (54) has at least countable or finite continuously differentiable solutions on $\left[t_{0}-c, t_{0}+c\right]$.

Proof. (Application of Lemma A). In addition to initial value problem (54), we also consider the integral equation of the form as

$$
x(t)=y_{0}+\int_{t_{0}}^{t} f(s, x(s)) \mathrm{d} s,
$$


and next write this as the operator equation $x=T(x)$, for $x \in M \subset X$, where $X:=$ $C\left(\left[t_{0}-c, t_{0}+c\right]\right), M=\left\{x \in X:\left\|x-y_{0}\right\| \leq b, \quad\right.$ and $\left.\quad\|x\|=\max _{t_{0}-c \leq t \leq t_{0}+c}|x(t)|\right\}$. The set $M$ is closed, convex, and bounded in $X$; also from this it follows $\bar{T}(M) \subset M$. Since the operator $T$ is compact,we have that Taskovic fixed point theorem (Theorem 46) implies the existence at least countable or finite solutions $x=T(x), x \in M$. The proof is complete.

Existence principle for systems of equations. As a simple application of the Brouwer fixed point theorem, we will prove an important existence statement for the system of the form as

$$
g_{i}(x)=0, \quad \text { for } \quad i=1, \ldots, n ;
$$

where $x=\left(\xi_{1}, \ldots, \xi_{n}\right) \in \mathbb{R}^{n}$. The key for solution of this problem is in the next boundary condition which we can briefly write in the following form as

$$
\sum_{i=1}^{n} g_{i}(x) \xi_{i} \geqslant 0 \text { for all } x \text { with }\|x\|=r .
$$

We notice that this existing problem will play a decisive role in the discussion of the Galerkin method for monotone operators, cf. Z e i d l e r [1990, p. 557].

Proposition 39. (Solution of system equations (55)). Let $C l K(0, r)=\left\{x \in \mathbb{R}^{n}\right.$ : $\|x\| \leq r\}$ for fixed $r>0$ and $x \mapsto\|x\|$ a norm on $\mathbb{R}^{n}$. Let $g_{i}: C l K(0, r) \rightarrow \mathbb{R}$ be continuous for $i=1, \ldots, n$. If (56) is satisfied, then (55) has at least countable or finite solutions $x$ with $\|x\| \leqslant r$.

Proof. (Application of Lemma A). Set $g(x)=\left(g_{1}(x), \ldots, g_{n}(x)\right)$ and suppose that $g(x) \neq 0$ for all $x \in \mathrm{Cl} K(0, r)$. Then define

$$
f(x)=-r g(x) /\|g(x)\|,
$$

and, now $f$ is continuous map of compact, convex set $\mathrm{Cl} K(0, r)$ into itself. By General Brouwer fixed point theorem (Proposition 44) there exist at least countable or finite fixed points $x=f(x)$. Taking norms, we see that $\|x\|=r$. Furthermore,

$$
\sum_{i=1}^{n} g_{i}(x) \xi_{i}=-r^{-1}\|g(x)\| \sum_{i=1}^{n} f_{i}(x) \xi_{i}=-r^{-1}\|g(x)\| \sum_{i=1}^{n} \xi_{i}^{2}<0,
$$

contrary to the preceding fact (56). Thus, we have that $g(x)=0$ for countable or finite $x \in \mathrm{Cl} K(0, r)$. The proof is complete.

3.2. An Extension Leray-Schauder Principle, Ta s k o v i ć [2005]. We will now show how to use topological methods, and in particular, the Schauder fixed point theorem for continuation with respect to $\lambda$-parameter. In this sense we have the following Leray-Schauder Principle in the form such as.

Theorem 26. (Leray-Schauder [1934], Tasković [2005]). Let X be a Banach space. Suppose that the operator $T: X \rightarrow X$ is compact and that there exists an $r>0$ such that

$$
x=\lambda T(x) \text { with } 0<\lambda<1 \quad \text { implies }\|x\| \leq r,
$$

then the equation of the form as $x=T(x)$ has at least countable or finite solutions. (Notice that (57) is trivially fulfilled if the following inequality holds as $\left.\sup _{x \in X}\|T(x)\|<\infty\right)$. 
Proof. (Application of Lemma A). Let $X$ be a Banach space and $T$ is a compact operator. We define an operator

$$
S(x)=\left\{\begin{array}{l}
T(x) \quad \text { if } \quad\|T(x)\| \leq 2 r, \\
\frac{2 r T(x)}{\|T(x)\|} \quad \text { if } \quad\|T(x)\|>2 r ;
\end{array}\right.
$$

and we claim that $S: M \rightarrow M$ is compact on $M:=\{x \in X:\|x\| \leq 2 r\}$. Obviously, $S$ is continuous. To establish compactness, let $\left\{x_{n}\right\}_{n \in \mathbb{N}}$ be a sequence in $M$. We consider two cases, namely there is: (a) a subsequence $\left\{y_{n}\right\}_{n \in \mathbb{N}}$ of $\left\{x_{n}\right\}_{n \in \mathbb{N}}$ such that $\left\|T\left(y_{n}\right)\right\| \leq 2 r$ for all $n \in \mathbb{N}$, and (b) a subsequence $\left\{y_{n}\right\}_{n \in \mathbb{N}}$ such that $\left\|T\left(y_{n}\right)\right\|>2 r$ for all $n \in \mathbb{N}$.

In case (a), the compactness of $T$ implies that there is a subsequence $\left\{z_{n}\right\}_{n \in \mathbb{N}}$ of $\left\{y_{n}\right\}_{n \in \mathbb{N}}$ such that $S\left(z_{n}\right)=T\left(z_{n}\right) \rightarrow y$ as $n \rightarrow \infty$.

In case (b), one can choose $\left\{z_{n}\right\}_{n \in \mathbb{N}}$ so that $1 /\left\|T\left(z_{n}\right)\right\| \rightarrow \alpha$ and $T\left(z_{n}\right) \rightarrow y$ as $n \rightarrow \infty$ for suitable $\alpha$ and $y$, so that $S\left(z_{n}\right) \rightarrow 2 r \alpha y$ as $n \rightarrow \infty$.

The Tasković fixed point theorem (Proposition 46) provides us with at least countable or finite $x \in M$ for which $S(x)=x$. If $\|T(x)\| \leqslant 2 r$, then $T(x)=$ $S(x)=x$. The other case, ||$T(x) \mid>2 r$, is impossible, for otherwise,

$$
S(x)=\lambda T(x)=x, \quad 0<\lambda=\frac{2 r}{\|T(x)\|}<1,
$$

which forces $\|x\|=2 r$, while the condition (57) requires that $\|x\| \leqslant r$. The proof is complete.

Annotations. We notice that by using Theorem 26 we can obtain an existence proof for the stationary Navier-Stokes equations (i.e., stationary movement of a viscous fluid).

Let $X$ be a nonempty set. The function $\rho: X \times X \rightarrow[0,+\infty]:=\mathbb{R}_{+}^{0} \cup\{+\infty\}$ is called a lower transverse on $X$ (or lower transversal) iff: $\rho[x, y]=\rho[y, x]$, $\rho[x, y]=+\infty$ if and only if $x=y$, and if there is a lower bisection function $\mu:[0,+\infty]^{2} \rightarrow[0,+\infty]$ such that

$$
\rho[x, y] \geq \min \{\rho[x, z], \rho[z, y], \mu(\rho[x, z], \rho[z, y])\}
$$

for all $x, y, z \in X$. A lower transversal space is a set $X$ together with a given lower transverse on $X$. The function $\mu$ in (Am) is called lower bisection function.

Annotation. For any nonempty set $S$ in the lower transversal space $X$ the diameter of $S$, denoted by $\operatorname{diam}(S)$, is defined as

$$
\operatorname{diam}(S):=\inf \{\rho[x, y]: x, y \in S\}
$$

where $A \subset B$ implies $\operatorname{diam}(B) \leqslant \operatorname{diam}(A)$. The relation $\operatorname{diam}(S)=+\infty$ holds if and only if $S$ is a one point set.

Elements of a lower transversal space will usually be called points. Given a lower transversal space $(X, \rho)$, with the bisection function $d:[0,+\infty]^{2} \rightarrow[0,+\infty]$ and a point $z \in X$, the open ball of center $z$ and radius $r>0$ is the set

$$
d(B(z, r))=\{x \in X: \rho[z, x]>r\} .
$$


On the other hand, from Tas k ovi ć [2005], the convergence $x_{n} \rightarrow x$ as $n \rightarrow \infty$ in the lower transversal space $(X, \rho)$ means that

$$
\rho\left[x_{n}, x\right] \rightarrow+\infty \text { as } n \rightarrow \infty,
$$

or equivalently, for every $\varepsilon>0$ there exist an integer $n_{0}$ such that the relation $n \geqslant n_{0}$ implies $\rho\left[x_{n}, x\right]>\varepsilon$.

The sequence $\left\{x_{n}\right\}_{n \in \mathbb{N}}$ in the lower transversal space $(X, \rho)$ is called transversal sequence (or lower Cauchy sequence) iff for every $\varepsilon>0$ there is an $n_{0}=n_{0}(\varepsilon)$ such that

$$
\rho\left[x_{n}, x_{m}\right]>\varepsilon \text { for all } n, m \geq n_{0} .
$$

Let $(X, \rho)$ be a lower transversal space and $T: X \rightarrow X$. We notice, from Taskovi ć [2005], that a sequence of iterates $\left\{T^{n}(x)\right\}_{n \in \mathbb{N}}$ in $X$ is said to be transversal sequence if and only if

$$
\lim _{n \rightarrow \infty}\left(\operatorname{diam}\left\{T^{k}(x): k \geq n\right\}\right)=+\infty .
$$

Also, a lower transversal space is called lower complete iff every transversal sequence converges. In this sense, a space $(X, \rho)$ is said to be lower orbital complete (or lower T-orbital complete) iff every transversal sequence which in contained in $\mathcal{O}(x):=\left\{x, T x, T^{2}(x), \ldots\right\}$ for some $x \in X$ converges in $X$.

Let $(X, \rho)$ be a lower transversal space and $T: X \rightarrow X$. We shall introduce the concept of LS-convergence in a space $X$; i.e., a lower transversal space $X$ satisfies the condition of LS-convergence (or $X$ is LS-complete) iff: $\left\{x_{n}\right\}_{n \in \mathbb{N}}$ is an arbitrary sequence in $X$ and $\sum_{i=1}^{\infty} \rho\left[x_{i}, x_{i+1}\right]=+\infty$ implies that $\left\{x_{n}\right\}_{n \in \mathbb{N}}$ has a convergent subsequence in $X$.

In connection with this, a lower transversal space $(X, \rho)$ satisfies the condition of orbital LS-convergence (or $X$ is orbital LS-complete) iff: $\left\{T^{n}(x)\right\}_{n \in \mathbb{N} \cup\{0\}}$ for $x \in X$ is an arbitrary iteration sequence in $X$ and

$$
\sum_{n=0}^{\infty} \rho\left[T^{n}(x), T^{n+1}(x)\right]=+\infty \quad(\text { for } x \in X)
$$

implies that $\left\{T^{n}(x)\right\}_{n \in \mathbb{N} \cup\{0\}}$ has a convergent subsequence in $X$.

We notice that in 2005 Tasković proved the following statement for a class of expansion mappings. Namely, if $(X, \rho)$ is an orbital $L S$-complete lower transversal space, if $T: X \rightarrow X$, and if there exists a number $q>1$ such that

$$
\rho(T(x), T(y)) \geqslant q \rho(x, y)
$$

for each $x, y \in X$, then $T$ has a unique fixed point in the lower transversal space $X$.

Let $\left(X, \rho_{X}\right)$ and $\left(Y, \rho_{Y}\right)$ be two lower transversal spaces and let $T: X \rightarrow Y$. We notice, from Task ovi ć [2005], that $T$ be lower transversal continuous (or lower continuous) at $x_{0} \in X$ iff for every $\varepsilon>0$ there exists a $\delta>0$ such that the relation

$$
\rho_{X}\left[x, x_{0}\right]>\delta \quad \text { implies } \quad \rho_{Y}\left[T(x), T\left(x_{0}\right)\right]>\varepsilon .
$$

A typical first example of a lower transversal continuous mapping is the mapping $T: X \rightarrow X$ with property (58). Also, the lower transverse $\rho$ needs not be lower transversal continuous; but, for an arbitrary metric function $r(x, y)$ the lower transverse of the form $\rho[x, y]:=1 / r(x, y)$ is a lower transversal continuous function. 
For further facts on the lower transversal continuous mapping see: Task ovi ć [2005].

We are now in a position to formulate our new statements in this part of the paper as Geometric statements of fixed point in the following forms.

Theorem 27. Let $T$ be a self-map on an orbital LS-complete lower transversal space $(X, \rho)$. Suppose that there exists a function $G: X \rightarrow \mathbb{R}_{+}^{0}$ such that

$$
\rho(x, T(x)) \geqslant G(T x)-G(x)
$$

for every $x \in X$. If $x \mapsto G(T x)$ is an upper semicontinuous function and if $G\left(T^{n} a\right) \rightarrow+\infty$ as $n \rightarrow \infty$ for some $a \in X$, then $T$ has at least countable or finite fixed points in $X$.

Proof (of a special case). Let $x \in X$ be an arbitrary point in $X$. We can show then that the sequence of iterates $\left\{T^{n} x\right\}_{n \in \mathbb{N} \cup\{0\}}$ in $X$ satisfies the condition of $L S$-convergence. In this sense, from $(\mathrm{Aw})$ we have

$$
\sum_{i=0}^{n} \rho\left[T^{i} x, T^{i+1} x\right] \geqslant G\left(T^{n+1} x\right)-G(x),
$$

and thus, from the conditions for functional $G$, as $n \rightarrow \infty$, we obtain an inequality and an equality as the following fact:

$$
\sum_{i=0}^{\infty} \rho\left[T^{i} a, T^{i+1} a\right] \geqslant \lim _{n \rightarrow \infty}\left(G\left(T^{n+1} a\right)-G(a)\right)=+\infty .
$$

Hence, for the subsequence of iterates $\left\{T^{n(k)} a\right\}_{k \in \mathbb{N} \cup\{0\}}$ in $X$, by orbital $L S$-completeness, there is $\xi \in X$ such that $T^{n(k)}(a) \rightarrow \xi(k \rightarrow \infty)$. Since $\xi \in X$, from (Aw), we obtain the following inequality in the form $\rho(\xi, T(\xi)) \geqslant G(T \xi)-G(\xi)$; i.e., since $x \mapsto G(T x)$ is an upper semicontinuous function, we have

$$
\rho(\xi, T(\xi)) \geqslant \limsup _{k \rightarrow \infty} G\left(T^{n(k)+1} a\right)-G(\xi)=+\infty ;
$$

which means, by the properties of a lower transversal space, $T \xi=\xi$. The proof is complete.

In connection with the preceding statement, we can now show that the following facts hold. Let $X$ be a topological space (or only a nonempty set), let $T: X \rightarrow X$ and let $A: X \times X \rightarrow \mathbb{R}_{+}^{0}$ be a given mapping. We shall introduce the concept of $L A$-variation in a space $X$, i.e., a topological space $X$ satisfies the condition of $L A$-variation iff: there exists a function $A: X \times X \rightarrow \mathbb{R}_{+}^{0}$ such that

$$
\sum_{n=0}^{\infty} A\left(T^{n}(x), T^{n+1}(x)\right)=+\infty
$$

for arbitrary $x \in X$. In this case if (59) holds, we say and that $T: X \rightarrow X$ is unbounded variation or unbounded $A$-variation.

In connection with the preceding statement, we can now show that the following facts hold from the following.

Lemma 4. Let $X$ be a nonempty set, $T: X \rightarrow X$, and let $A: X \times X \rightarrow \mathbb{R}_{+}^{0}$ be a given mapping. Then the following facts are mutually equivalent: 
(a) $T$ is an unbounded variation mapping on a nonempty set $X$ in the sense of the arbitrary given mapping $A$.

(b) There is an unbounded function $G: X \rightarrow \mathbb{R}_{+}^{0}$ such that holds in this sense the following inequality in the form as

$$
A\left(T^{n}(x), T^{n+1}(x)\right) \geqslant G(T x)-G(x)
$$

for every $n \in \mathbb{N} \cup\{0\}$ and for every $x \in X$, where $G\left(T^{n} x\right) \rightarrow+\infty$ as $n \rightarrow \infty$.

(c) There is a nonnegative sequences of real functions in the form $x \mapsto C_{n}(x, T x)$ such that the following inequality holds

$$
A\left(T^{n}(x), T^{n+1}(x)\right) \geqslant C_{n}(x, T x)
$$

for every $n \in \mathbb{N} \cup\{0\}$ and for every $x \in X$, where $\sum_{n=0}^{\infty} C_{n}(x, T x)=+\infty$ for arbitrary $x \in X$.

Proof. For the proof of this facts, first, suppose that it holds (a), then we define the function $G: X \rightarrow \mathbb{R}_{+}^{0}$ by

$$
G(x)=\sum_{i=0}^{n} A\left(T^{i} x, T^{i+1} x\right) \quad \text { for } \quad x \in X ;
$$

and thus we have $G(T x)-G(x) \leq A\left(T^{n} x, T^{n+1} x\right)$, i.e., the condition (b) holds. If (b) holds, then we obtain that the following inequality holds

$$
A\left(T^{n} x, T^{n+1} x\right) \geqslant G\left(T^{n+1} x\right)-G\left(T^{n} x\right)
$$

for every $x \in X$ and for every $n \in \mathbb{N} \cup\{0\}$. We set $C_{n}(x, T x)=G\left(T^{n+1} x\right)-G\left(T^{n} x\right)$ then directly we obtain (c). Also, elementary, (a) is a consequence of (c). The proof is complete.

Annotation. Let $X$ be a topological space let $T: X \rightarrow X$, and let $A: X \times X \rightarrow \mathbb{R}_{+}^{0}$ be a given mapping. We shall introduce the concept of LS-convergence in a space $X$, i.e., a topological space $X$ satisfies the condition of LS-convergence iff: $\left\{x_{n}\right\}_{n \in \mathbb{N}}$ is an arbitrary sequence in $X$ and $\sum_{i=1}^{\infty} A\left(x_{i}, x_{i+1}\right)=+\infty$ implies that $\left\{x_{n}\right\}_{n \in \mathbb{N}}$ has a convergent subsequence in $X$.

On the other hand, a topological space $X$ satisfies the following condition of orbital LS-convergence iff: $\left\{T^{n} x\right\}_{n \in \mathbb{N} \cup\{0\}}$ for $x \in X$ is an arbitrary iteration sequence in $X$ and $\sum_{n=0}^{\infty} A\left(T^{n} x, T^{n+1} x\right)=+\infty$ (for $x \in X$ ) implies that $\left\{T^{n} x\right\}_{n \in \mathbb{N} \cup\{0\}}$ has a convergent subsequence in $X$.

We are now in a position to formulate our main geometric statements of fixed point on arbitrary topological spaces.

Theorem 28. Let $T$ be a self-map on a topological space $X$ which is with the property of orbital LS-convergence. Suppose that there exists a function $G: X \rightarrow \mathbb{R}$ such that

$$
A(x, T(x)) \geqslant G(T x)-G(x)
$$

for every $x \in X$. If $x \mapsto G(T x)$ is an upper semicontinuous function and if $A(a, b)=+\infty$ iff $a=b$ and if $G\left(T^{n} z\right) \rightarrow+\infty(n \rightarrow \infty)$ for some $z \in X$, then $T$ has at least countable or finite fixed points in $X$. 
The proof of this statement is totally analogous with the proofs of the preceding statements of this type as an example in part 1 of chapter 3 of the book.

In connection with the preceding, we shall introduce the concept of lower topological space. In this sense, the function $A: X \times X \rightarrow[0,+\infty]$ is called a lower topological transverse on a nonempty set $X$ (or lower topological transversal) iff: $A(x, y)=+\infty$ if and only if $x=y$ for all $x, y \in X$. A lower topological space $X:=(X, A)$ is a topological space $X$ together with a given lower topological transverse $A$ on $X$.

Otherwise, the function $A$ is called a semilower topological transverse on a nonempty set $X$ iff: $A(x, y)=+\infty$ implies $x=y$ for all $x, y \in X$. A semilower topological space $X:=(X, A)$ is a topological space $X$ together with a given semilower topological transverse $A$ on $X$.

For further results the following fact is essential. In this sense, we notice that we can modify the preceding statement in the following sense. Namely, the next statement follows directly from Theorem 28 such as.

Theorem 28a. Let $T$ be a self-map on a $L S$-complete semi lower transversal space $(X, \rho)$. Suppose that there exists an unbounded above function $G: X \rightarrow \mathbb{R}_{+}^{0} \cup\{+\infty\}$ such that for any $x \in X$, with $x \neq T x$, there exists $y \in X \backslash\{x\}$ with property

$$
\rho(x, y) \geqslant G(y)-G(x),
$$

where $x \mapsto G(T x)$ is an upper semicontinuous function, then $T$ has at least countable or finite fixed points in $X$. (For further applications this statement is essential!)

A brief written suitable proof of this statement based on Lemma of Infinite Maximality may be found in the book by Task ovi ć [2005].

General annotation. For further facts, in connection with the preceding problems of fixed point and transversal spaces, see: T a s k o v i ć [2005].

Lower bounded linear operators. Let $X$ and $Y$ be lower transversal normed spaces over $\mathbb{K}$. The linear operator $A: X \rightarrow Y$ is called lower bounded (or bounded) iff

$$
\inf _{x \in X \backslash\{0\}} \frac{\|A x\|}{\|x\|}>0 ;
$$

and, thus, the set $B(X, Y)$ of lower bounded linear operators from $X$ to $Y$ together with the operator lower norm of the form

$$
\|A\|:=\inf _{x \in X \backslash\{0\}} \frac{\|A x\|}{\|x\|}
$$

is a complete (lower complete) lower transversal normed space over $\mathbb{K}$.

Let $X$ and $Y$ be lower transversal normed spaces, then a map $f: M \subset X \rightarrow Y$ is lower transversal continuous (or lower continuous) at $x \in M$ iff for every $\varepsilon>0$ there is a $\delta=\delta(\varepsilon)>0$ such that $\|f(x)-f(y)\|>\varepsilon$ whenever $y \in M$ and $\|x-y\|>\delta$.

Lower relative compactness. Let $(X,\|\cdot\|)$ be a lower transversal normed space. A set $M$ in $X$ is lower bounded iff there is a number $r>0$ such that $\|x\| \geqslant r$ for all $x \in M$.

A set $M$ in $X$ is lower relative compact (resp. lower compact) iff every sequence in $M$ contains a lower convergent subsequence (resp. the lower limit of which also belongs to $M$ ). 
Let $M \subset X$, the set of points $x_{1}, \ldots, x_{n(\varepsilon)} \in M$ is called a lower finite $\varepsilon$-net for $M$ iff $\min _{i=1, \ldots, n}\left\|x-x_{i}\right\|>\varepsilon$ for all $x \in M$. In this sense, $M$ is lower relative compact iff for every $\varepsilon>0$ there is a lower finite $\varepsilon$-net for $M$.

The set $M$ is lower dense in $X$ iff $\bar{M}=X$, i.e., for every $x \in X$ there exists a sequence $\left\{x_{n}\right\}_{n \in \mathbb{N}}$ in $M$ such that $x_{n} \rightarrow x$ as $n \rightarrow \infty$. This is equivalent to the condition that for every $x \in X$ and for every $\varepsilon>0$ there is a point $y \in M$ such that $\|x-y\|>\varepsilon$. The concept of denseness in lower transversal normed spaces covers many new approximation results.

Let $X$ and $Y$ be lower transversal normed spaces, then a map $f: M \subset X \rightarrow Y$ is lower transversal continuous (or lower continuous) at $x \in M$ iff $x_{n} \rightarrow x$ as $n \rightarrow \infty$ implies $f\left(x_{n}\right) \rightarrow f(x)$ as $n \rightarrow \infty$.

Here, it is naturally assumed that all of the $x_{n}$ belong to $M$. That $f$ is lower transversal continuous at $x$ then it is equivalent to: for every $\varepsilon>0$ there is a $\delta(\varepsilon)>0$ such that

$$
\|f(x)-f(y)\|>\varepsilon \quad \text { whenever } \quad y \in M \quad \text { and } \quad\|x-y\|>\delta(\varepsilon) .
$$

Further we will denote by $\mathcal{B}\left(\mathbb{R}_{+}^{0}\right)$ the set of all upper bisection functions $\psi$ : $\left(\mathbb{R}_{+}^{0}\right)^{2} \rightarrow \mathbb{R}_{+}^{0}$ which are increasing satisfying $\psi(t, t) \leq t$ for every $t \in \mathbb{R}_{+}^{0}$.

We will further on denote by $\mathcal{D}([0,+\infty])$ the set of all lower bisection functions $d:[0,+\infty]^{2} \rightarrow[0,+\infty]$ which are increasing, satisfying $d(t, t) \geqslant t$ for every $t \in$ $[0,+\infty]$.

Let $G$ be a nonempty bounded open set in $\mathbb{R}^{n}$. Then $L C(\bar{G})$ denotes the set of all real lower transversal continuous functions $f: \bar{G} \rightarrow \mathbb{R}$. For the special case where $G$ is a bounded open interval $(a, b)$ we write $L C[a, b]$ for $L C(\bar{G})$. In this sense, the set $M$ in $L C(\bar{G})$ is lower relatively compact iff: (i) (lower uniformly boundedness)

$$
\inf _{f \in M}\left(\frac{1}{\sup _{x \in \bar{G}}|f(x)|}\right)>0
$$

(ii) (lower equicontinuity) for every $\varepsilon>0$ there is a $\delta(\varepsilon)>0$ such that the following fact holds, that is

$$
\left(\sup _{f \in M}|f(x)-f(y)|\right)^{-1}>\varepsilon \quad \text { whenever } \quad x, y \in \bar{G} \quad \text { and } \quad|x-y|^{-1}>\delta(\varepsilon) ;
$$

where here $\delta(\epsilon)$ is independent of $x, y$ and $f$.

The space $L C(\bar{G}, Y)$. Let $G$ be a nonempty bounded open set in $\mathbb{R}^{n}$ and let $\left(Y,\|\cdot\|_{Y}\right)$ be a lower transversal normed space over $\mathbb{K}$. We let $L C(\bar{G}, Y)$ denote the set of all lower transversal continuous functions $f: \bar{G} \rightarrow Y$. Then $L C(\bar{G}, Y)$ becomes a lower transversal normed space over $\mathbb{K}$ with the maximum norm of the form $\|f\|=1 / \max _{x \in \bar{G}}\|f(x)\|_{Y}$.

In this sense we have the following statement of a Form of the Arzelà-Ascoli theorem for the lower transversal normed spaces: The set $M$ in $L C(\bar{G}, Y)$ is lower relatively compact iff: the set $\{f(x): f \in M\}$ is lower relatively compact in $Y$ for all $x \in \bar{G}$, and for every $x \in \bar{G}$ and every $\varepsilon>0$ there is a $\delta(\varepsilon, x)>0$ which is independent of the function $f$ such that

$$
\inf _{f \in M}\|f(x)-f(y)\|_{Y}>\varepsilon \quad \text { whenever } \quad y \in \bar{G} \quad \text { and } \quad\|x-y\|>\delta(\varepsilon, x) .
$$


A brief proof of this statement may be found in: Task ović [2005]. This statement is essential for further facts on lower transversal normed spaces.

3.3. Lower compact operators, T a s k o v i ć[2005]. Let $X$ and $Y$ be lower transversal normed spaces and $T: D(T) \subset X \rightarrow Y$ an operator. The operator $T$ is called lower compact iff: $T$ is lower transversal continuous and $T$ maps lower bounded sets into lower relatively compact sets.

Typical example of lower compact operators on infinite dimensional lower transversal normed spaces is integral operator in the following form:

$$
(T x)(t)=\int_{a}^{t} K(t, s, x(s)) d s \text { for all } t \in[a, b],
$$

where $K:[a, b] \times[a, b] \times[-r, r] \rightarrow \mathbb{K}(:=\mathbb{R}, \mathbb{C})$ is a continuous function. Set, further, that the following fact holds as

$$
M:=\{x \in L C([a, b], \mathbb{K}):\|x\| \geqslant r\}
$$

where $\|x\|=1 / \max _{a \leq s \leq b}|x(s)|$ and $L C([a, b], \mathbb{K})$ is the space of all lower transversal continuous maps $x:[a, b] \rightarrow \mathbb{K}$.

In this sense, we will consider $T$ for $\mathbb{K}=\mathbb{R}$. The remaining case is treated similarly. The set $A=[a, b] \times[a, b] \times[-r, r]$ is compact, whence $K$ is bounded and uniformly continuous on $A$. Thus, there is a number $\alpha$ such that $|K(t, s, x)| \leq \alpha$ for all $(t, s, x) \in A$, and for every $\varepsilon>0$ there is a $\delta(\varepsilon)>0$ such that $\mid K\left(t_{1}, s_{1}, x_{1}\right)-$ $K\left(t_{2}, s_{2}, x_{2}\right) \mid<\varepsilon$ for all $\left(t_{i}, s_{i}, x_{i}\right) \in A$ and $i=1,2$ satisfying $\left|t_{1}-t_{2}\right|+\left|s_{1}-s_{2}\right|+$ $\left|x_{1}-x_{2}\right|<\delta(\varepsilon)$. Let $z=T x$ and $x \in M$. Then

$$
\frac{1}{z(t)} \geqslant \frac{1}{\left|\int_{a}^{t} K(t, s, x(s)) d s\right|} \geqslant \frac{1}{(b-a) \alpha}
$$

for all $t \in[a, b]$. Furthermore, for $\left|t_{1}-t_{2}\right| \leqslant \min \{\delta(\varepsilon), \varepsilon\}$, we obtain the following suitable inequalities of the form

$$
\begin{gathered}
\frac{1}{\left|z\left(t_{1}\right)-z\left(t_{2}\right)\right|}=\left|\int_{a}^{t_{1}} K\left(t_{1}, s, x(s)\right) d s-\int_{a}^{t_{2}} K\left(t_{2}, s, x(s)\right) d s\right|^{-1}= \\
=\left|\int_{a}^{t_{1}}\left(K\left(t_{1}, s, x(s)\right)-K\left(t_{2}, s, x(s)\right)\right) d s-\int_{t_{1}}^{t_{2}} K\left(t_{2}, s, x(s)\right) d s\right|^{-1} \geqslant \\
\geqslant \frac{1}{(b-a) \varepsilon+\left|t_{1}-t_{2}\right| \alpha} \geqslant \frac{1}{((b-a)+\alpha) \varepsilon} ;
\end{gathered}
$$

and thus, the preceding two inequalities are uniformly true for $z=T x$ with arbitrary $x \in M$. By the Form of the Arzelà-Ascoli theorem (for lower transversal spaces), the set $T(M)$ is lower relative compact.

On the other hand, the operator $T$ is lower transversal continuous on $M$. To see this, let $\left\{x_{n}\right\}_{n \in \mathbb{N}}$ be a sequence in $M$ with $\left\|x_{n}-x\right\| \rightarrow+\infty$ as $n \rightarrow \infty$, i.e., the functions $x_{n}(t)$ lower converge uniformly on $[a, b]$ to $x(t)$. Set $z_{n}=T\left(x_{n}\right)$ and 
$z=T(x)$. Then

$$
\begin{gathered}
\left\|z-z_{n}\right\|=\frac{1}{\max _{a \leq t \leq b}\left|z(t)-z_{n}(t)\right|}= \\
\left.=\max _{a \leq t \leqslant b}\left|\int_{a}^{t}\left(K(t, s, x(s))-K\left(t, s, x_{n}(s)\right)\right) d s\right|\right)^{-1} \rightarrow+\infty \quad \text { as } n \rightarrow \infty ;
\end{gathered}
$$

and thus, all the preceding facts together imply the lower compactness of $T$. The prove of this fact is complete.

Convexity on lower transversal spaces. The set $C$ in linear space is lower convex if for $x, y \in C$ and $\lambda \in[1,2]$ implies that $\lambda x+(1-\lambda) y \in C$. The lower transversal space $(X, \rho)$ is called lower convex (or transversal lower convex) if for any two different points $x, y \in X$ there is a point $z \in X(z \neq x, y)$ such that

$$
\rho[x, y]+\rho[y, z]=2^{-1} 9 \rho[x, z] .
$$

In connection with this, if $C \subset X$ is a lower convex set of a transversal lower normed space $X$, then $C$ is also transversal lower convex space with $\rho[x, y]=1 /\|x-y\|$ for the classical norm $\|$.$\| , because for any two different points x, y \in C$ there is a point $z:=(3 y-x) / 2 \in C(z \neq x, y)$ such that $(\mathrm{Cd})$ holds. For further facts on lower transversal normed spaces see part 5 of this chapter of the book by Taskovic [2005].

We are now in a position to formulate the following general statements which are based for geometry (lower convexity) of lower transversal spaces. The following statements are the very connection with the famous Schauder's problem (Scottish book, problem 54), from: Tasković [2002].

Proposition 40. Let $C$ be a nonempty lower convex compact subset of a linear topological space $X$ and suppose $T: C \rightarrow C$ is a lower continuous mapping. Then $T$ has a fixed point in $C$.

A suitable brief proof of this statement may be found in Task ovic [2005]. We can give a proof of this statement also from the preceding facts of this book.

Proposition 41. Suppose that $C$ is a nonempty lower convex lower compact subset of $\mathbb{R}^{n}$, and that $T: C \rightarrow C$ is a lower continuous mapping. Then $T$ has a fixed point in $C$.

We can now formulate Proposition 41 in a manner valid for all transversal lower normed linear spaces.

Proposition 42. Let $C$ be a nonempty, lower compact, lower convex subset of a transversal lower normed space $X$, and suppose $T: C \rightarrow C$ is a lower continuous operator. Then $T$ has a fixed point in $C$.

We notice that Propositions 41 and 42 are direct consequences of Proposition 40. A brief suitable proof of Proposition 42 may be found in Tas k o v i ć [2005].

On the other hand, in connection with the preceding facts, the set $C$ in linear space is convex if for $x, y \in C$ and $\lambda \in[0,1]$ implies $\lambda x+(1-\lambda) y \in C$.

The lower transversal space $(X, \rho)$ is called L-convex (or transversal L-convex) if for two different points $x, y \in X$ there is a point $z \in X(z \neq x, y)$ such that

$$
\rho[x, y]+\rho[y, z]=\rho[x, z] .
$$


In connection with this, if $C \subset X$ is a convex set of a transversal lower normed space $X$, then $C$ is also transversal L-convex space with $\rho[x, y]=1 /\|x-y\|$ for the classical norm $\|$.$\| , because for any two different points x, y \in C$ there is a point

$$
z:=\frac{3-\sqrt{5}}{2} y+\frac{\sqrt{5}-1}{2} x \in C \quad(z \neq x, y)
$$

such that (Dc) holds. For further facts of this see: Ta s k o v i ć[2005].

Proposition 43. Let $C$ be a nonempty L-convex lower compact subset of a linear topological space $X$ and suppose $T: C \rightarrow C$ is a lower continuous mapping. Then $T$ has a fixed point in $C$.

We can give a proof of this statement from the preceding facts of this book. The proof of this statement is similar with the proof of the former Proposition 40.

Proposition 44. Suppose that $C$ is a nonempty L-convex lower compact subset of $\mathbb{R}^{n}$, and that $T: C \rightarrow C$ is a lower continuous mapping. Then $T$ has a fixed point in $C$.

From Proposition 43 we can now formulate Proposition 44 in a manner valid for all transversal lower normed linear spaces.

Proposition 45. Let $C$ be a nonempty, lower compact, $L$-convex subset of a transversal lower normed space $X$, and suppose $T: C \rightarrow C$ is a lower continuous operator. Then $T$ has a fixed point in $C$.

We notice that Propositions 44 and 45 are direct consequences of Proposition 43. A brief suitable proof of Proposition 45 may be found in T a s k o v i ć[2005].

Open problem 3. Does every lower continuous mapping of compact set $C \subset X$ into itself in linear topological space $X$ have a fixed point in $C$, where $C$ with the property that: $\lambda x+(1-\lambda) y \in C$ for all $x, y \in C$ and $\lambda \in[n, n+1]$ for an arbitrary fixed number $n \in \mathbb{N}$ !?

We notice that the cases $n=0$ and $n=1$, of this problem, are solved via Propositions 40 and 43 in this part of this chapter of the book.

Lemma 5. Let $(X, \rho)$ be a lower transversal space. If $C$ is a transversal $L$-convex or lower transversal convex set and if $T: C \rightarrow C$, then there exists a function $G: C \rightarrow \mathbb{R}_{+}^{0} \cup\{+\infty\}$ such that $T$ with the property $(L c)$.

Proof. Let $a \in C$ be a fixed element and let $x \in C$ be an arbitrary point with $x \neq a$. First, since $C$ is a $L$-convex set in $X$, it follows from definition that for $a \in C$ and for all $x \in C \backslash\{a\}$ there exists a point $y \neq a, x$ in $C$ such that $\rho[a, x]+\rho[x, y]=\rho[a, y]$. Hence, we also have the following inequality of the form

$$
3 \rho[x, y] \geqslant \rho[x, y]=\rho[a, y]-\rho[a, x],
$$

for every $x \in C \backslash\{a\}$. On the other hand, if $C$ is a transversal lower convex set, then again it holds (60) for every $x \in C \backslash\{a\}$. We notice that (60) simply holds and for $x=a$. Hence, from inequality (60) define function $G: C \rightarrow \mathbb{R}_{+}^{0} \cup\{+\infty\}$ such that

$$
G(x)=3^{-1} \rho[a, x] \quad \text { for } \quad x \in C
$$


Then, clearly, from (60) and (61) we have for any $x \in C$ that there exists $y \neq x$ in $C$ such that $\rho[x, y] \geqslant G(y)-G(x)$. Thus, for any $x \in C$ with $x \neq T x$ there exists $y \in C \backslash\{x\}$ such that is (Lc). Hence, it follows that $T$ is with the property (Lc) and the proof is complete.

Lemma 6. Let $X$ be a linear space. If $C$ is a L-convex or lower convex set in $X$ and if $T$ is a map of $C$ into itself, then there exists a continuous function $G: C \rightarrow \mathbb{R}_{+}^{0} \cup\{+\infty\}$ such that $T$ is a with the property (Lc).

Proof. Consider the $L$-convex (or lower convex) set $C$ of the linear space $X$ as a lower quasi-transversal space with the quasi lower transverse $q$, where $q: C \times C \rightarrow$ $\mathbb{R}_{+}^{0} \cup\{+\infty\}$ defined by

$$
q(x, y)=\left\{\begin{array}{l}
+\infty, \quad \text { for } x=y, \\
\min \{K(x), K(y)\}, \text { for } x \neq y
\end{array}\right.
$$

for a strictly convex continuous function $K: C \rightarrow \mathbb{R}_{+}^{0} \cup\{+\infty\}$. Then it is easy to see that $q$ is a lower quasi-transverse, i.e., that for all $x, y, z \in C$ we have: $q(x, y)=q(y, x)$, and

$$
q(x, y) \geqslant \min \{q(x, z), q(z, y), d(q(x, z), q(z, y))\}
$$

for some $d:\left(\mathbb{R}_{+}^{0} \cup\{+\infty\}\right)^{2} \rightarrow \mathbb{R}_{+}^{0} \cup\{+\infty\}$, and that $x=y$ implies $q(x, y)=+\infty$. On the other hand, if $q(x, y)=+\infty$ and $x \neq y$, i.e., if $K(x)=K(y)=+\infty$, then since $K$ is a strictly convex function, we obtain the following fact

$$
+\infty=\frac{K(x)+K(y)}{2}>K\left(\frac{x+y}{2}\right)=+\infty,
$$

which is a contradiction. Consequently, $x=y=(x+y) / 2$, i.e., $x=y$. Thus, $q(x, y)=+\infty$ implies $x=y$, i.e., $q$ is a continuous lower transverse on $C$.

Applying Lemma 5 to this case, we obtain then that there exists a continuous function $G: C \rightarrow \mathbb{R}_{+}^{0} \cup\{+\infty\}$ defined by $G(x)=3^{-1} q(x, y)$ such that $T$ is with the property $(\mathrm{Lc})$. The proof is complete.

Proof of Propositions 40 and 43. From Lemma 6 and the preceding facts there exists a continuous function $G: C \rightarrow \mathbb{R}_{+}^{0} \cup\{+\infty\}$ such that $T$ is with the property (Lc). Since $T$ is a lower continuous mapping, the function $x \mapsto G(T x)$ is an upper semicontinuous function. The set $C$ is a compact in the space $X$ and thus $C$ satisfies the condition of $L S$-completeness. It is easy to see that $T$ satisfies all the required hypotheses in Theorem 28a. Hence, it follows from Theorem 28a, that $T$ has at least countable or finite fixed points in $C$. The proof is complete.

In connection with this, we notice that a direct equivalent translation of Propositions 40 and 43 to lower compact operators is the following result.

Proposition 46. Let $C$ be a nonempty, closed, lower bounded, L-convex (or lower convex) subset of a lower complete transversal lower normed space $X$, and suppose $T: C \rightarrow C$ is a lower compact operator. Then $T$ has at least countable or finite fixed points in $C$.

A brief proof of this statement may be found in Tas k ovi ć [2005]. This form of these statement is useful for applications! 
Existing facts for Systems of Equations. As a simple application of the Proposition 46, we will prove an important existence statement for the system

$$
g_{i}(x)=0, \quad \text { for } \quad i=1, \ldots, n \text {; }
$$

where $x=\left(\xi_{1}, \ldots, \xi_{n}\right) \in \mathbb{R}^{n}$. The key is in the boundary condition of the following form which we write in the suitable form as

$$
\sum_{i=1}^{n} g_{i}(x) \xi_{i} \geqslant 0, \quad \text { for all } x \text { with }\|x\|=r .
$$

Proposition 47. [Solution of $(\mathrm{Se})]$. Let $x \mapsto\|x\|$ be a lower norm on $\mathbb{R}^{n}$ with homogenity $\|\lambda x\|=|\lambda|\|x\|$ or $\|\lambda x\|=|\lambda|^{-1}\|x\|$, and let $C l K(0, r)=\left\{x \in \mathbb{R}^{n}\right.$ : $\|x\| \geqslant r\}$ for fixed $r>0$. If $g_{i}: C l K(0, r) \rightarrow \mathbb{R}$ is lower transversal continuous for $i=1, \ldots, n$ and if $(\mathrm{Bc})$ is satisfied, then (Se) has at least countable or finite solutions $x \in \mathbb{R}^{n}$ with $\|x\| \geqslant r$.

Proof. Set $g(x)=\left(g_{1}(x), \ldots, g_{n}(x)\right)$ and suppose that $g(x) \neq 0$ for every $x \in C l K(0, r)$. Then, in the case $\|\lambda x\|=|\lambda|\|x\|$, define the following function of the form $f(x)=-r g(x) /\|g(x)\|$. Now $f$ is a lower transversal continuous map of the lower compact, $L$-convex set $C l K(0, r)$ into itself. By Proposition 44 there exists a fixed point $x=f(x)$. Taking lower norms, we see that $\|x\|=r$. Furthermore,

$$
\sum_{i=1}^{n} g_{i}(x) \xi_{i}=-\frac{1}{r}\|g(x)\| \sum_{i=1}^{n} f_{i}(x) \xi_{i}=-\frac{1}{r}\|g(x)\| \sum_{i=1}^{n} \xi_{i}^{2}<0,
$$

contrary to (Bc); thus, the statement holds. In the second case, if $\|\lambda x\|=|\lambda|^{-1}\|x\|$, then, we define the following function in the form as

$$
f(x)=-\frac{\|g(x)\|}{r} g(x) ;
$$

and thus, now $f$ is a lower transversal continuous map of the lower compact, $L$ convex set $C l K(0, r)$ into itself. Again, by Proposition 46, there exists a fixed point $x=f(x)$. Taking lower norms, we see that $\|x\|=r$. Furthermore,

$$
\sum_{i=1}^{n} g_{i}(x) \xi_{i}=-\frac{r}{\|g(x)\|} \sum_{i=1}^{n} f_{i}(x) \xi_{i}=-\frac{r}{\|g(x)\|} \sum_{i=1}^{n} \xi_{i}^{2}<0,
$$

contrary to $(\mathrm{Bc})$; and thus, the statement holds in this case. The proof is complete.

We notice that on a different manner new proofs may be given for Propositions 43, 44 and 45 (as well as for Propositions 41 and 42) via an approximation process for lower compact operators. In this sense we have the following result.

Proposition 48. (Approximation for Lower Compact Operators). Let $X$ and $Y$ be lower complete lower transversal normed spaces with a bisection function $d \in \mathcal{D}([0,+\infty])$ and with homogenity $\|h x\|=|h|\|x\|$ or $\|h x\|=|h|^{-1}\|x\|$ and let $M$ be a nonempty lower bounded subset of $X$. If $T: M \subset X \rightarrow Y$ is a given operator, then $T$ is lower compact if and only if for every $n \in \mathbb{N}$ there exists a lower compact operator $P_{n}: M \rightarrow Y$ such that

$$
\inf _{x \in M}\left\|T(x)-P_{n}(x)\right\| \geqslant n
$$


and $\operatorname{ldim}\left(\operatorname{lspan} P_{n}(M)\right)>0$, where $\|\cdot\|$ is an arbitrary continuous lower norm on $X$ and $Y$, and where ldim and lspan are the suitable lower dim and lower span.

Proof. First, in the case of homogentity form $\|h x\|=|h|\|x\|$, let $T$ be lower compact. Then $T(M)$ is lower relative compact, so for each $n \in \mathbb{N}$ there exist elements $y_{i} \in T(M)$ for $i=1, \ldots, m$ such that

$$
\min _{i=1, \ldots, m}\left\|T(x)-y_{i}\right\|>\frac{n \sum_{i=1}^{m} h_{i}(x)}{\min \left\{h_{1}(x), \ldots, h_{m}(x)\right\}} \quad \text { for all } \quad x \in M
$$

from the preceding facts on lower relative compactness and from the following definition that we have the following fact as

$$
P_{n}(x)=\frac{\sum_{i=1}^{m} h_{i}(x) y_{i}}{\sum_{i=1}^{m} h_{i}(x)}
$$

where $h_{i}(x)=\max \left\{n-\left\|T(x)-y_{i}\right\|, 0\right\}$ has all the requisite properties, for the lower continuous $h_{i}(x)$ "do not all vanish simultaneously" for $x \in M$ by (63), and where by the generalized $(\mathrm{Nl})$, i.e., $(\mathrm{Nl}$ ')

$$
\begin{gathered}
\left\|P_{n}(x)-T(x)\right\|=\left\|\left(\sum_{i=1}^{m} h_{i}(x)\right)^{-1} \sum_{i=1}^{m} h_{i}(x)\left(T(x)-y_{i}\right)\right\|= \\
=\left(\sum_{i=1}^{m} h_{i}(x)\right)^{-1}\left\|\sum_{i=1}^{m} h_{i}(x)\left(T(x)-y_{i}\right)\right\| \geqslant \cdots \geqslant \\
\geqslant\left(\sum_{i=1}^{m} h_{i}(x)\right)^{-1} \min \left\{\left\|h_{1}(x)\left(T(x)-y_{1}\right)\right\|, \ldots,\left\|h_{m}(x)\left(T(x)-y_{m}\right)\right\|\right\}= \\
=\left(\sum_{i=1}^{m} h_{i}(x)\right)^{-1} \min \left\{h_{1}(x)\left\|\left(T(x)-y_{1}\right)\right\|, \ldots, h_{m}(x)\left\|\left(T(x)-y_{m}\right)\right\|\right\} \geqslant n
\end{gathered}
$$

for all $x \in M$. The lower boundedness of $T(M)$ implies the lower boundedness of $P_{n}(M)$. Since the set $P_{n}(M)$ lies in a finite dimensional space, $P_{n}(M)$ is lower relative compact, i.e., the operator $P_{n}$ is lower compact.

In the case of homogenity form $\|h x\|=|h|^{-1}\|x\|$ let $T$ be lower compact. Then $T(M)$ is lower relative compact, so for each $n \in \mathbb{N}$ there exist elements $y_{i} \in T(M)$ for $i=1, \ldots, m$ such that

$$
\min _{i=1, \ldots, m}\left\|T(x)-y_{i}\right\|>\frac{n \sum_{i=1}^{m} h_{i}(x)}{\min \left\{1 / h_{1}(x), \ldots, 1 / h_{m}(x)\right\}} \quad \text { for all } \quad x \in M,
$$


from the preceding facts on lower relatively compact and from (64). Thus, by (Nl) or $\left(\mathrm{Nl}^{\prime}\right)$, as in the preceding case, we obtain

$$
\begin{aligned}
& \left\|P_{n}(x)-T(x)\right\|=\left\|\left(\sum_{i=1}^{m} h_{i}(x)\right)^{-1} \sum_{i=1}^{m} h_{i}(x)\left(T(x)-y_{i}\right)\right\| \geqslant \\
& \geqslant\left(\sum_{i=1}^{m} h_{i}(x)\right)^{-1} \min \left\{\left\|h_{1}(x)\left(T(x)-y_{1}\right)\right\|, \ldots,\left\|h_{m}(x)\left(T(x)-y_{m}\right)\right\|\right\}= \\
& =\left(\sum_{i=1}^{m} h_{i}(x)\right)^{-1} \min \left\{\frac{1}{h_{1}(x)}\left\|\left(T(x)-y_{1}\right)\right\|, \ldots, \frac{1}{h_{m}(x)}\left\|\left(T(x)-y_{m}\right)\right\|\right\} \geqslant n
\end{aligned}
$$

for all $x \in M$. The lower boundedness of $T(M)$ implies the lower boundedness if $P_{n}(M)$. Again, since the set $P_{n}(M)$ lies in a finite dimensional space, $P_{n}(M)$ is lower relatively compact, i.e., the operator $P_{n}$ is lower compact in this case, too.

On the other hand, suppose that (62) is true. As the lower uniform limit of lower continuous operators $P_{n}$, the operator $T$ is itself lower continuous, for by (62) and $(\mathrm{Nl})$ we have

$$
\|T(x)-T(y)\| \geqslant \cdots \geqslant \min \left\{n,\left\|P_{n}(x)-P_{n}(y)\right\|, n\right\}>3 \varepsilon
$$

for sufficiently large fixed $n \in \mathbb{N}$ and $\|x-y\|>\delta(\varepsilon)$. Furthermore, $T(M)$ is lower relative compact, since (62) implies that for each $n \in \mathbb{N}$, the set $T(M)$ has a lower finite $n / 2$-net. Note that the lower relative compact set $P_{n}(M)$ has a $n$-net. The proof is complete.

A principle of continuation. We will now show how to use topological methods for the variant of Proposition 46 for continuation with respect to parameter $\lambda$ for the lower compact operators.

Proposition 49. (Principle of Continuation, Tasković [2005]). Let $X$ be a lower complete transversal lower normed space with homogenity $\|h x\|=|h|\|x\|$ or $\|h x\|=$ $|h|^{-1}\|x\|$ and suppose that the operator $T: X \rightarrow X$ is lower compact and there exists an $r>0$ such that

$$
x=\lambda T(x) \text { with } \lambda>1 \text { implies }\|x\| \geqslant 3 r,
$$

then the equation $x=T(x)$ has at least countable or finite solutions. (Notice that (Co) is trivially fulfilled if the following inequality holds in the form as $\inf _{x \in X}$ $\|T(x)\|>0$.)

Proof. In the case of homogenity form $\|h x\|=|h|\|x\|$ we define an operator of the following form as

$$
S(x)=\left\{\begin{array}{l}
T(x) \quad \text { if } \quad\|T(x)\| \geqslant 2 r, \\
\frac{2 r T(x)}{\|T(x)\|} \quad \text { if } \quad\|T(x)\|<2 r
\end{array}\right.
$$

where we claim that $S: M \rightarrow M$ is lower compact on $M:=\{x:\|x\| \geqslant 2 r\}$. Obviously, $S$ is lower transversal continuous. 
To establish lower compactness, let $\left\{x_{n}\right\}_{n \in \mathbb{N}}$ be a sequence in $M$. We consider two cases: namely, there is a subsequence $\left\{a_{n}\right\}_{n \in \mathbb{N}}$ of $\left\{x_{n}\right\}_{n \in \mathbb{N}}$ such that $\left\|T\left(a_{n}\right)\right\| \geqslant$ $2 r$ for all $n \in \mathbb{N}$; and there is a subsequence $\left\{a_{n}\right\}_{n \in \mathbb{N}}$ such that $\left\|T\left(a_{n}\right)\right\|<2 r$ for all $n \in \mathbb{N}$.

In the first case, the lower compactness of $T$ implies that there is a subsequence $\left\{b_{n}\right\}_{n \in \mathbb{N}}$ of $\left\{a_{n}\right\}_{n \in \mathbb{N}}$ such that $S\left(b_{n}\right)=T\left(b_{n}\right) \rightarrow b$ as $n \rightarrow \infty$.

In the second case, one can choose $\left\{b_{n}\right\}_{n \in \mathbb{N}}$ so that $1 /\left\|T\left(b_{n}\right)\right\| \rightarrow a$ and $T\left(b_{n}\right) \rightarrow$ $b$ as $n \rightarrow \infty$ for suitable $a$ and $b$, so that $S\left(b_{n}\right) \rightarrow 2 r a b$ ad $n \rightarrow \infty$.

In this context, the Proposition 46 provides us with at least countable or finite $x \in M$ for which $S(x)=x$. If $\|T(x)\| \geqslant 2 r$, then $T(x)=S(x)=x$. The other case, $\|T(x)\|<2 r$, is impossible, for otherwise,

$$
S(x)=\lambda T(x)=x, \quad \lambda=\frac{2 r}{\|T(x)\|}>1,
$$

which forces $\|x\|=2 r$, while this case requires $\|x\| \geqslant 3 r$. In the case of homogenity form $\|h x\|=|h|^{-1}\|x\|$ we define an operator of the following form

$$
S(x)=\left\{\begin{array}{l}
T(x) \text { if }\|T(x)\| \geqslant 2 r, \\
\frac{\|T(x)\| T(x)}{2 r} \text { if }\|T(x)\|<2 r ;
\end{array}\right.
$$

where we claim that $S: M \rightarrow M$ is lower compact on $M$. Obviously, $S$ is lower transversal continuous. Further proof is totally analogous with the preceding case.

Finally, the Proposition 46 provides us with an $x \in M$ for which $S(x)=x$. If $\|T(x)\| \geqslant 2 r$, then $T(x)=S(x)=x$. The other case, $\|T(x)\|<2 r$, is impossible, for otherwise,

$$
S(x)=\lambda T(x)=x, \quad \lambda=\frac{2 r}{\|T(x)\|}>1,
$$

which forces $\|x\|=2 r$, while this case requires $\|x\| \geqslant 3 r$. The proof is complete.

Remarks. This result of Proposition 49 is in a direct connection with the famous Leray-Schauder Principle of Continuation for compact operators in 1934 .

One of the earliest successes of functional analysis dates from 1916 when $\mathrm{F}$. $\mathrm{R}$ i e s z used linear space methods to prove some of Fredholm's results on linear integral equations.

The concept of a normed linear space had not been formulated in 1916, and Riesz worked with integral equations, but his techniques generalize directly and can be applied to a special case of linear operators, now called compact operators.

Now we shall show how Proposition 42 and 45, i.e., Proposition 46, can be applied for investigation of solvability of the initial value problem of the form

$$
x^{\prime}(t)=f(t, x(t)), \quad x\left(t_{0}\right)=y_{0},
$$

where $f$ is merely continuous or lower transversal continuous. In this sense the following result holds.

Theorem 29. Let there be given real numbers $t_{0}$ and $y_{0}$ and the following suitable set as a rectangle of the form

$$
Q_{b}:=\left\{(t, x) \in \mathbb{R}^{2}:\left|t-t_{0}\right| \leq 1 / a,\left|x-y_{0}\right| \leq 1 / b\right\},
$$


where $a$ and $b$ are fixed positive numbers. Suppose that $f: Q_{b} \rightarrow \mathbb{R}$ is continuous or lower transversal continuous and bounded with

$$
|f(t, x)| \leq K \quad \text { for all }(t, x) \in Q_{b},
$$

and fixed $K>0$. For $m=\min \{1 / a, 1 / b K\}$ then the initial value problem (De) has at least countable or finite lower transversal continuous differentiable solutions on the closed interval of the form $\left[t_{0}-m, t_{0}+m\right]:=I$.

In further let $L C\left[t_{0}-m, t_{0}+m\right]$ denote the space of all lower transversal continuous functions $x(t)$ where the lower norm $\|\cdot\|$ in the form $\|x\|=1 / \max _{t \in I}|x(t)|$.

Proof of Theorem 29. For the proof of this statement we first replace (De) by the integral equation in the form

$$
x(t)=y_{0}+\int_{t_{0}}^{t} f(s, x(s)) d s,
$$

and next write this as the operator equation $x=T x$ for $x \in M \subset X$, where $M=\left\{x \in X:\left\|x-y_{0}\right\| \geqslant b\right\}$.

The set $M$ is closed, lower bounded, and $L$-convex of transversal lower normed space $X:=L C\left[t_{0}-m, t_{0}+m\right]$. We notice that $T(M) \subset M$, i.e., $T$ maps $M$ into $M$. For if $x \in M$, then $\left\|x-y_{0}\right\| \geqslant b$, and hence $\left|x(t)-y_{0}\right| \leq 1 / b$ for all $t \in I$. Thus,

$$
\left\|T x-y_{0}\right\|=\frac{1}{\max _{t \in I}\left|\int_{t_{0}}^{t} f(s, x(s)) d s\right|} \geqslant \frac{1}{m K} \geqslant b ;
$$

therefore, $T x \in M$. The operator $T: M \rightarrow M$ is lower compact by the preceding typical example. Now, Proposition 46 implies the existence of a solution $x=T x$, $x \in M$. The proof is complete.

In this part, also, our goal is to generalize the preceding Form of Peano's theorem to equations of the form

$$
x^{\prime}(t)=f(t, x(t)), \quad x\left(t_{0}\right)=y_{0},
$$

where $x(t)$ lies in a lower transversal normed space $Y$. In the special case where $Y=\mathbb{R}^{p},(65)$ is the system

$$
\xi_{i}^{\prime}(t)=f_{i}(t, x(t)), \quad \xi_{i}\left(t_{0}\right)=\eta_{i 0}, \quad i=1, \ldots, n ;
$$

where $x(t)=\left(\xi_{1}(t), \ldots, \xi_{n}(t)\right)$, of $n$ ordinary differential equations. For continuous $f_{i}$ we obtain the equivalent system of integral equations

$$
\xi_{i}(t)=\eta_{i 0}+\int_{t_{0}}^{t} f_{i}(s, x(s)) d s, \quad i=1, \ldots, n .
$$

As our lower transversal normed space we choose the set of all $x(t)$ for which the components $\xi_{i}(t)$ are lower continuous on $\left[t_{0}-m, t_{0}+m\right]$. For the lower norm on $Y$ we use $\|x\|_{Y}:=1 / \max _{1 \leq i \leq n}\left(\max _{a \leqslant t \leq b}\left|\xi_{i}(t)\right|\right)$, then in complete analogy with Theorem 29 one shows the existence of solutions for some $m$ and for continuous $f_{i}$ in the class of all lower continuous functions.

In contrast to former fact that $f$ is continuous, or lower transversal continuous, we now require $f$ to be a lower compact mapping. 
Theorem 30. (Generalized Theorem 29). Let $Y$ be a lower complete lower transversal normed space with a bisection function $d \in \mathcal{D}([0,+\infty])$, let $t_{0} \in \mathbb{R}$ and $y_{0} \in Y$, and

$$
Q_{b}:=\left\{(t, y) \in \mathbb{R} \times Y:\left|t-t_{0}\right| \leq 1 / a,\left\|y-y_{0}\right\|_{Y} \leqslant 1 / b\right\},
$$

for fixed numbers $0<a, b<\infty$. Suppose that $f: Q_{b} \rightarrow Y$ is a lower compact map and that $\|f(t, y)\|_{Y} \leq K$ for all $(t, y) \in Q_{b}$ with fixed $K>0$. If we set $m=\min \{1 / a, 1 / b K\}$, then (65) has at least countable or finite lower continuously differentiable solutions on $\left[t_{0}-m, t_{0}+m\right]:=I$.

Proof. We set $X:=L C\left(\left[t_{0}-m, t_{0}+m\right], Y\right)$ for $0<m<+\infty$, i.e., $X$ is the space of all lower continuous functions $x:\left[t_{0}-m, t_{0}+m\right] \rightarrow Y$, where as a lower norm, we choose

$$
\|x\|_{X}:=\max _{t \in\left[t_{0}-m, t_{0}+m\right]}\|x(t)\|_{Y}
$$

where $\|x\|_{Y}:=\left(\max _{t \in I}|x(t)|\right)^{-1}$ and, where $M:=\left\{x \in X:\left\|x-y_{0}\right\|_{X} \geqslant b\right\}$ is a ball (lower) in $X$. Define $z(t)$ to be the right side of the integral equation

$$
x(t)=y_{0}+\int_{t_{0}}^{t} f(s, x(s)) d s:=z(t),
$$

and set $T(x)=z$. Then, this equation corresponds to the fixed point problem $x=T(x)$ for $x \in M \subset X$, and is also equivalent to (65). Since $\left\|x-y_{0}\right\|_{X} \geqslant b$ and $\left\|z-y_{0}\right\|_{X} \geqslant 1 / m K \geqslant b$ and

$$
\left\|z\left(t_{1}\right)-z\left(t_{2}\right)\right\|_{Y} \geqslant \frac{1}{K\left|t_{1}-t_{2}\right|}
$$

for all $t_{1}, t_{2} \in\left[t_{0}-m, t_{0}+m\right]$; if we now set

$$
A(t):=y_{0}+\left(t-t_{0}\right) \overline{\operatorname{conv}}\left\{f(s, x(s)): s \in\left[t_{0}-m, t_{0}+m\right]\right\},
$$

where $\overline{\text { conv }}$ denote closed convex hull, then $z(t) \in A(t)$ and $T(M) \subset M$.

The set $T(M)$ is lower relative compact in $X$, because for (67) it shows that the functions $z \in T(M)$ are lower uniform continuous functions, and for all $t \in$ $\left[t_{0}-m, t_{0}+m\right]$, their values lie in the lower compact set $A(t)$. Then, the Form of Arzelà-Ascoli theorem implies that $T(M)$ is lower relative compact.

On the other hand, the operator $T$ is lower continuous on $M$. To see this, let $x_{n} \rightarrow x$ in $X$ as $n \rightarrow \infty$. By (50), we have

$$
\left\|T x_{n}-T x\right\|_{X} \geqslant m \inf _{s \in\left[t_{0}-m, t_{0}+m\right]}\left\|f\left(s, x_{n}(s)\right)-f(s, x(s))\right\|_{Y} \rightarrow+\infty
$$

as $n \rightarrow \infty$. For if it did not, there would be an $\varepsilon_{0}>0$ and a sequence, denoted for brevity by $\left(s_{n}\right)$ in $\left[t_{0}-m, t_{0}+m\right]$ for which

$$
\left\|f\left(s_{n}, x_{n}\left(s_{n}\right)\right)-f\left(s_{n}, x\left(s_{n}\right)\right)\right\|_{Y} \leqslant \varepsilon_{0}
$$


then there is a subsequence, again denoted by $\left(s_{n}\right)$ and an $s_{0}$ such that $s_{n} \rightarrow s_{0}$ as $n \rightarrow \infty$, and

$$
\begin{aligned}
& \left\|x_{n}\left(s_{n}\right)-x\left(s_{0}\right)\right\|_{Y} \geqslant \min \left\{\left\|x_{n}\left(s_{n}\right)-x\left(s_{n}\right)\right\|_{Y},\left\|x\left(s_{n}\right)-x\left(s_{0}\right)\right\|_{Y},\right. \\
& \left.d\left(\left\|x_{n}\left(s_{n}\right)-x\left(s_{n}\right)\right\|_{Y},\left\|x\left(s_{n}\right)-x\left(s_{0}\right)\right\|_{Y}\right)\right\} \geqslant \\
& \geqslant \min \left\{\left\|x_{n}\left(s_{n}\right)-x\left(s_{n}\right)\right\|_{Y},\left\|x\left(s_{n}\right)-x\left(s_{0}\right)\right\|_{Y},\right. \\
& \left.\min \left\{\left\|x_{n}\left(s_{n}\right)-x\left(s_{n}\right)\right\|_{Y},\left\|x\left(s_{n}\right)-x\left(s_{0}\right)\right\|_{Y}\right\}\right\}= \\
& =\min \left\{\left\|x_{n}\left(s_{n}\right)-x\left(s_{n}\right)\right\|_{Y},\left\|x\left(s_{n}\right)-x\left(s_{0}\right)\right\|_{Y}\right\} \rightarrow+\infty \quad \text { as } \quad n \rightarrow \infty \text {. }
\end{aligned}
$$

In this sense, we note that $\left\{x_{n}(s)\right\}_{n \in \mathbb{N}}$ is lower uniformly convergent, $x(t)$ is lower continuous, and $f$ is lower continuous, therefore both $f\left(s_{n}, x_{n}\left(s_{n}\right)\right)$ and $f\left(s_{n}, x\left(s_{n}\right)\right)$ lower converge to $f\left(s_{0}, x\left(s_{0}\right)\right)$ as $n \rightarrow \infty$, contradicting (68).

Apply the Proposition 46 to obtain the existence of a fixed point of $T$ on $M$. The proof is complete.

\section{REFERENCES}

[1] S. Banach, Sur l'équation fonctionnelle, Fund. Math., 1 (1920), 123-124.

[2] S. Banach, Sur les opérations dans les ensembles abstraits et leur application aux équations intégrales, Fund. Math., 3 (1922), 133-181.

[3] S. Banach, Sur les fonctionnelles linéaires I-II, Studia Math., 1 (1929), 211-216 i 223-239.

[4] S. Banach, Théorie des opérations linéaires, Monografie Matematyczne, t.1, Varsovie, 1932.

[5] H. Hahn, Über lineare Gleichungssysteme in linearen Räumen, J. Reine Angew. Math., 157 (1926), 214-229.

[6] F. Riesz, Untersuchungen über Systeme integrierbarer Funktionen, Math. Ann., 69 (1910), 449-497.

[7] F. Riesz, Über lineare Funktionalgleichungen, Acta Math., 41 (1918), 71-98.

[8] F. Riesz, and B. Sz. Nagy, Functional Analysis, Ungar, New York, 1955.

[9] E. Zermelo, Beweis dass jede Menge wohlgeordnet werden kann, (Aus einem an Herrn Hilbert gerichteten Briefe), Math. Ann., 59 (1904), 514-516.

[10] E. Zermelo, Neuer Beweis für die Möglichkeit einer Wohlordnung, Math. Ann., 65 (1908), 107-128.

[11] M. Zorn, A remark on method in transfinite algebra, Bull. Amer. Math. Soc., 41 (1935), 667-670.

[12] M. Zorn, Idempotency of infinite cardinals, Univ. Calif. Publ. in Math., Seminar Reports (Los Angeles), 2 (1944), 9-12.

[13] M. R. Tasković, Theory of transversal point, spaces and forks, Fundamental Elements and Applications, Monographs of a new mathematical theory, VIZ-Beograd 2005, (In Serbian), 1054 pages. English summary: 1001-1022.

[14] M. R. Tasković, The Axiom of Infinite Choice, Math. Moravica, 16 (2012), 77-94.

Remark. The complete list of the references of this paper is from the preceding book by Tasković [2005]. 
Milan R. TAsković

FaCulty of Mathematics

P.O. Box 550

11000 BEOGRAD

SERBIA

Home address:

Milan R. TAsković

Nehruova 236

11070 BELGRAdE

Serbia

E-mail address: andreja@predrag.us 